DOI: $10.1002 /$ admi.201601118

Article type: Review

\title{
In situ characterization of interfaces relevant for efficient photo-induced reactions
}

Oliver Supplie, Matthias M. May, Sebastian Brückner, Nadzeya Brezhneva, Thomas

Hannappel*, and Ekaterina V. Skorb*

Dr. O. Supplie, Dr. S. Brückner, Prof. Dr. T. Hannappel

Technical University Ilmenau, Institute for Physics, Ilmenau, Germany

E-mail: thomas.hannappel@tu-ilmenau.de

Dr. M.M. May

University of Cambridge, Department of Chemistry, Cambridge, UK

N. Brezhneva, Dr. E.V. Skorb

Laboratory of Solution Chemistry of Advanced Materials and Technologies (SCAMT), ITMO

University, St. Petersburg, Russian Federation

E-mail: skorb@mpikg.mpg.de

Keywords: interfaces, energy conversion, in situ analysis, biosensors, photo-induced reactions

Abstract:

Solar energy conversion and photo-induced bioactive sensors are representing topical scientific fields, where interfaces play a decisive role for efficient application. The key to specifically tune these interfaces is a precise knowledge of interfacial structures and their formation on the microscopic, preferably atomic scale. Gaining thorough insight into interfacial reactions, however, is particularly challenging in relevant complex chemical environment. This review introduces a spectrum of material systems with corresponding interfaces significant for efficient applications in energy conversion and sensor technologies. We highlight appropriate analysis techniques capable of monitoring critical physico-chemical reactions in situ during non-vacuum preparation and photoactivity studies including welldefined inorganic epitaxial reference surfaces, buried interfaces and low-defect nucleation of disjunct epitaxial materials that are analyzed during preparation in chemical vapor environment. Their surfaces are then modified and functionalized in the gaseous and liquid environment. Finally, we review even more complex coupling of inorganic stable photoactive materials with responsive soft matter for bioactivity. Interface formation, structure and/or artificial photochemical interfacial reactions are scrutinized down to the atomic scale in real time, also accounting for equilibrium versus non-equilibrium, kinetically driven processes, in order to accelerate progresses in the realization of efficient energy materials and in the exploitation of photo-induced processes at interfaces. 


\section{Introduction}

In his Nobel lecture, H. Kroemer concisely coined the famous phrase "Often it can be said, that the interface is the device" ${ }^{[1]}$ This is particularly true for the semiconductor heterostructures which he pioneered and established. In general, interfaces do not only determine electronic properties of the final device; their atomic order also highly affects growth kinetics and defect formation. Moreover, the design of solid-liquid and hybrid interfaces determines their chemical reactivity and stability. In situ monitoring of interface preparation, formation and interfacial reactions thus promises efficient process control. Paired with a detailed understanding of interface formation mechanisms, however, the true power of in situ control is to allow for specific modification and tuning of interface formation for the device of choice. There are several excellent reviews on in situ approaches covering wide ranges of materials from basic science to applications as well as varieties of preparation and analysis techniques. ${ }^{[2,3,4,5,6,7,8,9,10,11]}$ As indicated in Fig. 1, this review will be focused on in situ control over interfaces of materials that are relevant for photo-induced reactions in high-efficiency solar energy conversion and sensing applications with in situ control of semiconductor / polymer / biological interfaces. We will restrict the techniques to realistic and complex, non-ultrahigh-vacuum (UHV) ambient, where in situ control is most demandingbut also highly desired. The more complex interfacial reactions are, the more important is in situ characterization. Ex situ approaches can contribute to an indirect understanding of interface formation, but to understand and, finally, control the dynamic processes taking place, real-time measurements are appropriate. The challenge, we are facing, is twofold: On the one hand, increased interaction with the surrounding ambient causes higher complexity. Yet at the same time, it decreases the number of applicable techniques. On the other hand, those techniques are often elaborate and not necessarily easy to interpret. In a nutshell, we will discuss four main topics:

(i) Surface preparation during growth of structures for high-efficiency solar energy conversion: World-record conversion efficiencies in both photovoltaics ${ }^{[12,13]}$ and solar water splitting ${ }^{[14]}$ are achieved with multi-junction solar cells based on epitaxial III/V compound semiconductor structures. We will discuss in situ controlled preparation of relevant III/V and IV(100) surfaces in metalorganic vapor phase epitaxy (MOVPE). ${ }^{[15]}$ MOVPE is the state-of-the-art industrially scalable technique for semiconductor epitaxy. Compared to vacuum-based techniques, MOVPE is demanding given the involved complex physico-chemical reactions and the presence of a carrier gas, which limits in situ methods mainly to optical approaches.

(ii) Preparation of buried interfaces during growth of structures for high-efficiency solar energy conversion: Based on the homoepitaxially prepared surfaces, we will discuss MOVPE in situ studies on multinary III/V compounds, their related buried heterointerfaces - such as tunnel junctions in multi-junction solar cells - as well as on III/V-on-IV heteroepitaxy.

(iii) Solid-liquid interfaces and electrochemical interface modification: The study of catalytic and electrochemical processes requires high spatial as well as temporal resolution. A great challenge for in situ techniques is the necessity to transport information through the liquid environment. Materials involved range from catalytically active metals, semiconductors and photoelectrochemical applications to insulators used in surface passivation. Besides interfaces involving the III/V 
structures discussed above, some metallic and oxide-based materials and their interaction with gas-phase and liquid water will be reviewed.

(iv) Semiconductor / polymer / biological interfaces for the development of spatiotemporal nanoscale machinery inspired by nature: Here, we review the coupling of inorganic stable photoactive materials with responsive soft matter for bioactivity. We discuss inspiration by nature as a general design concept for chemical networks - which consist of several inter-related single chemical reactions - for application in future "intelligent" systems. Lab-produced, artificial photochemical networks are discussed to provide reliable, inorganic semiconductors for new functions. Thus we focus on a transformation of energy from electromagnetic irradiation into ion concentration gradients with in situ temporal control of the reaction network. Light is discussed here as an "on-demand" stimulation of the bioresponse, for which it is easy to control intensity, duration, and localization. Efficient actuation of $\mathrm{pH}$-sensitive soft assemblies with light is shown as an example.

For the first part (section 2), we will start from well-defined epitaxially prepared interfaces, such as InP(100) films and surfaces, which are relevant for high-efficiency solar energy conversion. The atomic order of these surfaces and their specific preparation in vacuum and MOVPE ambient has been studied and is now understood in great detail. They are perfectly suited to demonstrate the ideal interplay between in situ spectroscopy, density functional theory, and benchmarking to a broad range of surface science techniques. Based on their optical fingerprints, different atomically well-ordered surfaces can be specifically prepared. Following this approach, in situ control over surface preparation will be discussed for several important III/V compound semiconductor surfaces, such as GaAs and GaP, as well as for Ge and Si.

Building on these results, III/V materials will be combined to multinary compounds and III/V heterostructures, which are studied with focus on their (buried) interfaces in the second part of this review (section 3). This is of particular interest for semiconductor applications, where the sharpness of the heterointerface is of essential importance: Tunnel junctions will be addressed as well as heterointerfaces designed to suppress recombination of charge carriers. Appropriate interface preparation is also crucial for III/V heteroepitaxy on $\mathrm{Si}$ substrates, where crystal defects are easily introduced during III/V nucleation due to the different crystal structures involved. Here, we will demonstrate how in situ analysis enables detailed insights regarding the interface formation, which finally yields precise process control and fine interfacial tuning.

The structures discussed in section 2 and 3 are decisive for highly efficient solar energy conversion. Besides their applications in photovoltaic devices, they are promising ingredients for direct solar water splitting. ${ }^{[14,16,17,18]}$ Corresponding modifications of their surfaces in liquid environments and by gases beyond MOVPE conditions will therefore be discussed in the third part of this review (section 4 and 5). The impact of these complex conditions on the surfaces is understood to a much lesser degree. Adsorption processes on well-defined surfaces, the focus of section 4 , try to bridge the gap between epitaxial surfaces in vacuum and in liquid ambient: While for the former, the application of various surface science techniques enabled an understanding on an atomistic level, this is much more challenging for the latter due to a limited availability of applicable techniques. Here, we will first review studies on epitaxial reference surface discussed in part one, and secondly on noble metal surfaces. The next step directed towards applications for artificial photosynthesis in a water splitting device is to study the solid-liquid interface, which is the topic in section 5. Emphasis will be put on how 
an electrochemical in situ modification enabled record solar-to-hydrogen efficiencies and how this approach may be transformed to other surfaces.

In the fourth part of this review (section 6 to 9), we will discuss nature inspired photochemical reactions beyond applications of artificial photosynthesis: Photoinduced interfacial reactions also enable sensing applications focusing on modulation, detection, and deactivation of bacteria, pathogens as well as biofilms and biomolecules. They also serve as platforms for systems for microfluidic biochips and autonomous soft robotics. Photochemical reactions to control hybrid interfaces will be detailed in section 6. Different techniques for in situ detection of reactive oxygen species in $\mathrm{TiO}_{2}$-based systems are discussed in section 7 . This is followed by studies on the photocatalytic degradation of organic species on $\mathrm{TiO}_{2}$ in section 8. Finally, section 9 focuses on prospects for in situ modulation of soft matter and microorganisms without degradation.

\section{Epitaxial reference surfaces}

The attractiveness of the III/V semiconductor material class for a wide range of electro-optical applications is to a great extent owed to the possibility to smoothly tune their electronic structure via the composition of multinary compounds. Fig. 2 shows bandgaps and band offsets of the classical III/V semiconductors as a function of the lattice constant at room temperature and displays their approximated band alignment with respect to vacuum. The tunability of the bandgap allows absorption of a wide range of the solar spectrum. The combination of different materials enables high solar energy conversion efficiency: For a dual absorber structure, the limiting conversion efficiencies with dependence of the band gaps of the subcells are plotted in Fig. 3. The combination of III/V semiconductors with Si here promises efficiencies close to optimum, both for PV and water splitting application. ${ }^{[16,18,19,20]}$ Such a tandem absorber device requires preparation of ideal structures in all involved interfaces, which are manifold as indicated in Fig. 4 for an InP-based solar cell optimized for the infrared. ${ }^{[21,22]}$ It is therefore important to understand the surface formation step by step. We will thus first focus on surfaces of the important III/V semiconductors InP, GaP, and GaAs(100) as well as on the group IV substrates $\mathrm{Si}(100)$ and $\mathrm{Ge}(100)$. These are relevant for energy applications and are available as wafers, which facilitates the preparation of smooth and high-quality reference surfaces on homoepitaxially grown buffer layers.

MOVPE processing ambient hinders the application of electron-based techniques, so that mostly optical techniques are applied in situ. In the following, the linear optical technique reflection anisotropy spectroscopy ${ }^{[23]}$ (RAS) will play an important role. RAS measures the difference in (complex) reflection along two mutually perpendicular crystal axes normalized to their mean reflection,

$$
\mathbb{C} \ni \quad \frac{\Delta r}{r}=\frac{r_{x}-r_{y}}{1 / 2\left(r_{x}+r_{y}\right)} .
$$

Both real and imaginary parts can be measured, but mostly the real part $(R e)$ is discussed here. In literature, reflectance, $R$, is discussed often instead of the complex Fresnel reflection amplitude coefficient, $r$. For $\Delta r \ll r$, the relation of RAS (complex number, $r$ ) and the corresponding reflectance anisotropy signal (real number, $R$ ) is ${ }^{[23]}$

$$
\frac{\Delta R}{R} \approx 2 \operatorname{Re}\left(\frac{\Delta r}{r}\right) .
$$

For (100) faces of ideal cubic crystals the bulk contribution is optically isotropic which renders RAS an extremely interface and defect sensitive optical technique. Here, RAS is aligned such that $x=[0 \overline{1} 1]$ and $y=[011]$. To make RAS amplitudes intercomparable, they should be calibrated to a $\mathrm{Si}(110)$ reference. ${ }^{[23]}$ 
Contributions to RA spectra can be manifold, which renders RAS a versatile but also complicated analysis technique. Once the microscopic origin of the spectra is understood, they can act as qualitative and/or quantitative fingerprints for specific interfacial or defectrelated properties. In order to understand the origin of the spectra, comparison to to complementary surface science techniques ('benchmarking') is beneficial. Of course, such benchmarking should not affect the interface which is characterized, so that - besides various other optical techniques applicable in MOVPE ambient-UHV based techniques are often applied. Most elegantly, samples are transferred contamination-free from MOVPE ambient to a mobile UHV shuttle, ${ }^{[24]}$ which may in principle be attached to any UHV surface science chamber of interest, where the samples can be characterized in vacuo. Since RAS is also applicable in UHV, the state of the sample can be probed and verified after benchmarking as well.

\subsection{InP(100)}

InP has a long history as efficient photocathode in water splitting applications. ${ }^{[25,26]}$ Recently, InP-based low band-gap tandem solar cells were suggested for multi junctions solar cells (MJSC) with more than three junctions ${ }^{[27,28,29]}$ and applied in the current world-record four junction cell with conversion efficiencies exceeding 46\%. ${ }^{[13,30,31]}$ Here, $\operatorname{InP}(100)$ is of particular interest, since it may be considered as prototype surface regarding H-based MOVPE processing of phosphides, where hydrogen strongly affects the atomic surface structure compared to UHV preparation. InP(100) is also a valuable example for the fruitful combination of optical in situ spectroscopy, various complementary in system surface science techniques and density functional theory (DFT) calculations, which lead to a very detailed understanding of the atomic and electronic surface structure. The atomic structure indeed has a significant impact on further surface functionalization, as will be discussed in section 5 .

\section{Surface reconstructions and their anisotropic fingerprints}

Depending on the $\mathrm{P}$ chemical potential (corresponding to Ga- vs. P-rich conditions), two surface reconstructions typically occur in MOVPE ambient (cf. Fig. 5) and they exhibit characteristically different RAS fingerprints (cf. Fig. 6): (i) The P-rich $(2 \times 2) / c(2 \times 4)$ surface consists of buckled $\mathrm{P}$ dimers stabilized by one $\mathrm{H}$ atom each, ${ }^{[32]}$ while (ii) the In-rich $(2 \times 4)$ surface is terminated by a mixed dimer atop an In layer. ${ }^{[33]}$ Fig. 5 shows ball-and-stick models of these reconstructions and they are highlighted in the phase diagram in Fig. 6(b), which was obtained by DFT calculations. ${ }^{[32]}$ Fig. 6(a) displays the corresponding RA spectra obtained after homoepitaxial buffer growth and contamination-free transfer to UHV, ${ }^{[2]}$ where they were measured at $20 \mathrm{~K}$ : The P-rich surface (violet) features a characteristic minimum $P 1$ at about $1.9 \mathrm{eV}$, an intense maximum $P 2$ at $3.2 \mathrm{eV}$ slightly below the $\mathrm{E}_{1}$ interband transition, a broad local maximum $P 3$ between 4.0 and $4.5 \mathrm{eV}$ as well as an additional peak at the E0' interband transition. The RA spectrum of the In-rich InP(100) surface (green) exhibits more features, the most prominent are two local minima around $2.0 \mathrm{eV}$ (labelled Inl), a local maximum $\operatorname{In} 2$ at about $2.4 \mathrm{eV}$, a local minimum $\operatorname{In} 3$ close to the $\mathrm{E}_{1}$ interband transition, a rather broad local maximum In 4 centered at about $3.7 \mathrm{eV}$ and another local maximum In 4 at the $\mathrm{E}_{0}$ ' interband transition. In particular the spectral differences between $P 2$ and $\operatorname{In} 2 / \operatorname{In} 3$ are suitable for in situ identification of the two surfaces. The dielectric function of $\mathrm{InP}^{[34]}$ and its temperature dependence was measured in situ with ellipsometry, ${ }^{[35]}$ which facilitates evaluation at real growth conditions.

Within the DFT-GW approximation, reasonable agreement of calculated and in situ RA spectra both for the In-rich ${ }^{[36,37]}$ and the P-rich ${ }^{[32]} \operatorname{InP}(100)$ surface is achieved. The predicted spectral features help to clarify the microscopic origin of the RA spectra, which are best measured at low temperature, ${ }^{[36,38,39]}$ where peaks sharpen drastically. ${ }^{[35,40]}$ However, so 
far, the accuracy of DFT calculations is not sufficiently precise to predict RA spectra without any experimental feedback. Only in few specific cases it is possible to deduce the given atomic surface structure from experimental in situ RA spectra by comparison to theoretical ones.

\section{The P-rich InP(100) surface}

The P-rich InP(100) surface is an instructive example how the correlation of DFT and RAS enabled understanding the impact of the presence of $\mathrm{H}$ during MOVPE preparation: in situ RA spectra of both $\mathrm{PH}_{3}{ }^{[35,41]}$ and tertiarybutylphosphine (TBP) ${ }^{[42]}$ prepared P-rich $\operatorname{InP}(100)$ surfaces show a characteristic lineshape that does not occur in $\mathrm{H}$-free molecular beam epitaxy (MBE) ambient. ${ }^{[32,43]}$ DFT calculations could relate the features $P 1$ and $P 2$ to optical transitions involving states induced by $\mathrm{H}$ termination of one $\mathrm{H}$ atom per $\mathrm{P}$ dimer. ${ }^{[32]}$ The buckled $\mathrm{P}$ dimers of this " $(2 \times 1)-$ like" $(2 \times 2)-2 \mathrm{D}-2 \mathrm{H}$ reconstructed surface ${ }^{[32]}$ form zig-zag lines and the buckling may flip causing a $(2 \times 2) / \mathrm{c}(2 \times 4)$ symmetry. ${ }^{[44,45]}$ While dimer buckling may occur also on non H-terminated surfaces, ${ }^{[46]}$ which would cause streaked (2x1)-like low energy electron diffraction (LEED) patterns just as for the H-terminated buckled $\mathrm{P}$ dimers, the existence of the P-H bonds was verified experimentally by in system Fourier transform infrared spectroscopy (FTIR) studies. ${ }^{[4]}$ These studies also showed that RAS allows for in situ fine tuning of the atomic order at the $\mathrm{InP}(100)$ surface: ${ }^{[47]}$ The P-rich surface is commonly prepared by cooling under stabilization with the precursor TBP after homoepitaxial growth. When TBP supply is stopped at $300{ }^{\circ} \mathrm{C}$, the surface is covered with excess $\mathrm{P}$ and precursor residuals. ${ }^{[48]}$ Cycled heating to $360{ }^{\circ} \mathrm{C}$ (without precursor supply) and cooling to $300{ }^{\circ} \mathrm{C}$ (with precursor supply despite for the very last cooling step) increases the atomic order at the surface, ${ }^{[47]}$ which can be observed in situ by an increased intensity of $P 1$ along with a redshift of its energetic position. ${ }^{[42,48]}$ In situ studies during InP:adsorbate interaction revealed that the $\mathrm{H}$ termination strongly increases the stability of the P-rich surface against $\mathrm{O}_{2}$, yet not $\mathrm{H}_{2} \mathrm{O}$, ${ }^{[49}$, ${ }^{50]}$ as will be discussed more detailed in section 4 .

\section{The In-rich $\operatorname{InP(100)}$ surface}

Given the different lineshape of the anisotropic fingerprints of the two surface reconstructions, RAS enables to study the transformation from P-rich to In-rich surfaces in great detail: ${ }^{[48,51]}$ Heating of the P-rich surface above $370{ }^{\circ} \mathrm{C}$ without $\mathrm{P}$ stabilization results in enhanced $\mathrm{P}$ desorption and $\mathrm{P}$ depletion of the surface. ${ }^{[48]}$ RAS peaks originating from the occurrence of $\mathrm{P}$ dimers vanish and the lineshape changes towards that of the In-rich surface. Fig. 7 visualizes that the peaks at and below $2.5 \mathrm{eV}$ of the RA spectrum of the In-rich surface can be attributed to transitions involving surface states related to the mixed dimer reconstruction. ${ }^{[36,37]}$ The electronic orbitals for the corresponding surface states are shown in side view in Fig. 7.

\section{Experimental verification of the microscopic origin of the spectral features}

Further experimental indications for the origin of the contributions to the RA spectra of P-rich and In-rich $\operatorname{InP}(100)$ was obtained by analyzing their temperature-dependent phonon coupling: ${ }^{[40]}$ Fitting this temperature dependence with an adequate model ${ }^{[52]}$ yields the renormalization energy for each anisotropic contribution. ${ }^{[40]}$ For the peaks $P 1$ and $P 2$ as well as $\operatorname{In} 3$, the renormalization energy is similar to that of the InP bulk critical point energies, which implies an "intrinsic" ${ }^{[23]}$ nature. Since the $\mathrm{E}_{1}$ interband transition is close to $P 2$ and $I n 3$, it was suggested that these anisotropies likely stem from surface modified bulk transitions. ${ }^{[40]}$ $P 1$, in contrast, which is not in the vicinity of any critical interband transition, was attributed to a transition involving both bulk and surface states. ${ }^{[40]}$ Inl couples only weakly to phonons and was thus attributed to pure surface state transitions. ${ }^{[40]}$ 
The electronic structure of MOVPE-prepared InP(100) surfaces has been studied with in system ultraviolet photoelectron spectroscopy (UPS) ${ }^{[38]}$ and with angular-resolved photoelectron spectroscopy (ARPES) after contamination-free transfer ${ }^{[24]}$ to the BESSY-I synchrotron. ${ }^{[53]}$ Occupied surface states with only little dispersion close to the valence band maximum (VBM) as well as occupied surface resonances were identified. ${ }^{[53]}$ Performing in system 2-photon photoemission (2PPE) studies on In-rich $\operatorname{InP}(100),{ }^{[54]}$ an occupied surface resonance was found about $0.2 \mathrm{eV}$ below the VBM and two unoccupied dangling bond surface states were identified at $1.5 \mathrm{eV}$ and at $2.2 \mathrm{eV}$ above the VBM near the $\Gamma$-point. The alignment of these states relative to the VBM agrees well with that of the surface states $\mathrm{V}_{1,2}$, $\mathrm{C}_{1}$ and $\mathrm{C}_{2}$ predicted by DFT. ${ }^{[36]}$ Electron dynamics of $\mathrm{InP}(100)$ and scattering from surface to bulk states were studied applying pump-probe $2 \mathrm{PPE}$ at fs timescale. ${ }^{[55,56,57]}$

As first observed for GaAs (see below), doping influences the RA spectrum of $\operatorname{InP}(100)$ via the linear electro-optic effect. ${ }^{[58]}$

\section{2. $\mathrm{GaP}(100)$}

$\mathrm{GaP}$ is interesting for water splitting applications since its rather large bandgap of about 2.3 $\mathrm{eV}$ at room temperature might provide enough photovoltage ${ }^{[59]}$ However, due to this large bandgap and its indirect nature, thin GaP films are rather transparent and are thus not suitable as absorber layer for high efficiency solar energy conversion. However, GaP may be applied as window layer ${ }^{[60]}$ in tandem configurations, as constituent of multinary compounds such as InGaP or GaAsP or when diluted with $\mathrm{N}$ and possibly As. ${ }^{[61]}$ Moreover, GaP is almost latticematched to $\mathrm{Si}$, which renders it an ideal candidate for pseudomorphic virtual III/V-on-Si substrates, as will be discussed in section 3 .

$\mathrm{GaP}(100)$ surfaces reconstruct analogously to the $\operatorname{InP}(100)$ surfaces discussed afore and also their optical in situ fingerprints are similar. ${ }^{[62]}$ In H-based ambient, two surface reconstructions are typical: the P-rich surface (which occurs during growth and at rather low temperatures or when stabilized with TBP, respectively) and the Ga-rich surface (which forms when $\mathrm{P}$ desorbs preferentially at elevated temperatures). Fig. 8 compares the RA spectra of Prich $\mathrm{GaP}$ and Ga-rich $\mathrm{GaP}(100)$, which were prepared by MOVPE and measured in system at $20 \mathrm{~K} .{ }^{[63]}$ The spectrum of the P-rich surface (orange) exhibits a characteristic minimum $P 1$ at about $2.6 \mathrm{eV}$, an intense maximum $P 2$ at $3.7 \mathrm{eV}$ slightly below the $\mathrm{E}_{1}$ interband transition, a small local maximum $P 3$ at about $4.5 \mathrm{eV}$ as well as an local maximum $P 4$ at about $5 \mathrm{eV}$. The RA spectrum of the Ga-rich $\mathrm{GaP}(100)$ surface (blue) exhibits a characteristic minimum Gal slightly below $2.5 \mathrm{eV}$, a local maximum $G a 2$ at about $3.2 \mathrm{eV}$, a local minimum $G a 3$ close to the $\mathrm{E}_{1}$ interband transition, a shoulder $\mathrm{Ga} 4$ at about $4.2 \mathrm{eV}$ and a local maximum Ga5 slightly above the E0' interband transition. Compared to the RA spectra of the corresponding $\operatorname{InP}(100)$ surfaces, ${ }^{[38]}$ those of GaP are shifted to higher energies. While the lineshape of the P-rich surfaces are very similar, Gal is broader but exhibits less features than Inl.

In situ RA spectra of the Ga-rich $\mathrm{GaP}(100)$ surface were studied in combination with ab initio DFT calculations: ${ }^{[64]}$ A mixed Ga-P dimers on top of a layer was suggested as ground state just as for In-rich $\operatorname{InP}(100)$. The surface reconstruction is $(2 \times 4)$ as confirmed by LEED on decapped ${ }^{[62]}$ and contamination-free transferred ${ }^{[63]}$ homoepitaxial surfaces. The corresponding surface state band structure exhibits several non-resonant surface states both in the valence and the conduction band. ${ }^{[37]}$ Transitions involving these states cause the contributions to the RA spectrum below the $\mathrm{E}_{1}$ interband transition: As predicted by DFT, ${ }^{[37]}$ a series of occupied and unoccupied surface states was found experimentally applying in system $2 \mathrm{PPE}^{[65]}$ and transitions involving them were assigned to $G a 1 .{ }^{[37,65]}$ The presence of several anisotropic optical transitions can also explain the rather broad lineshape of Gal compared to Pl. These 
spectral features vanish when the surface is exposed to oxygen and the surface states are quenched. ${ }^{[65]}$ As we will see in section 4, however, the surface states exhibit a much larger stability against oxidation by water. Peak $G a 2$ stems from a surface modified bulk transition. $^{[37]}$

For the P-rich $\mathrm{GaP}(100)$ surface, it was shown by ab initio DFT calculations, ${ }^{[66]}$ that the lineshape of $P I$ can be explained well by optical transitions involving surface states from the buckled, H-stabilized P dimer. These states and the corresponding RAS signals induced by optical transitions between occupied and non-occupied surface states are shown in Fig. 9. The presence of a surface state assigned to $P 1$ was also verified experimentally with in system 2PPE. ${ }^{[65]}$ Just as for P-rich $\operatorname{InP}(100)$, the buckling of the dimer leads to a $(2 \times 2) / c(4 \times 2)$ surface reconstruction (also referred to as $(2 \times 2)-2 \mathrm{D}-2 \mathrm{H}^{[66]}$ ), which causes streaks in the LEED patterns ${ }^{[63]}$ and zig-zag lines observed by STM. ${ }^{[45,63,66,67]}$ At room temperature, the $\mathrm{H}$ atom is mobile and a flip flop motion of the dimer can be detected by STM. ${ }^{[45]}$

Continuously measured RA spectra during annealing of the $\mathrm{GaP}(100)$ surface in $\mathrm{H}$ ambient showed that $\mathrm{P}$ starts desorbing preferentially at temperatures beyond about $490{ }^{\circ} \mathrm{C}$ and that this causes a change from the P-rich to the Ga-rich surface reconstruction via an intermediate surface. ${ }^{[63,68]}$ In contrast to $\operatorname{InP}(100)$, surface preparation in $\mathrm{N}_{2}$-based ambient significantly impacts the surface formation. ${ }^{[51]}$ Upon annealing in $\mathrm{N}_{2}$, an additional intermediate surface phase occurs at temperatures above about $470{ }^{\circ} \mathrm{C}$ and below about $620{ }^{\circ} \mathrm{C} .{ }^{[51]}$

\section{3. $\operatorname{GaAs}(100)$}

GaAs is widely used in optoelectronics and it is the material of choice for record efficiency single junction solar cells ${ }^{[12,69]}$ as well as modules. ${ }^{[12,70]} \mathrm{GaAs}$ was used as active substrate in tandem absorber structures, ${ }^{[71,72]}$ and due to the little lattice mismatch, growth of GaAs-based top absorber structures on $\mathrm{Ge}(100)$ are applied in the current industry standard InGaP/GaAs/Ge solar cells, achieving more than $40 \%$ conversion efficiency. ${ }^{[73,74,75,76]} \mathrm{GaAs}$ also is the first compound semiconductor that was grown epitaxially by MOVPE ${ }^{[15]}$ and GaAs(100) surfaces were the first semiconductor surfaces studied in situ during MOVPE growth. ${ }^{[77]}$ These first experiments studied growth kinetics and chemisorption with time resolved RAS. ${ }^{[77][78]}$ RA spectra obtained in MOVPE ambient were often benchmarked to MBE-prepared GaAs(100) surfaces, where electron-based techniques are available. ${ }^{[78,79,80]}$ Besides RAS, surface photo-absorption (SPA) was applied in situ to compare the decomposition of the two typical precursors trimethylgallium (TMGa) and triethylgallium (TEGa). ${ }^{[1]}$ Applying in situ RAS on GaAs(100), surface reconstructions of semiconductors were observed for the first time in atmospheric pressure demonstrating the potential of and the need for real time growth monitoring due to complex surface structures and reactions. ${ }^{[82,83,84]}$ Surface reconstructions similar to those known from UHV studies were found to occur also for MOVPE-preparation in various process gases, ${ }^{\left[{ }^{82]}\right.}$ but, in general, surface reactions are more complex in MOVPE ambient. ${ }^{[85]}$ In particular, surface reactions play a crucial role during initiation of GaAs homoepitaxy. ${ }^{[79,80,86,87]}$

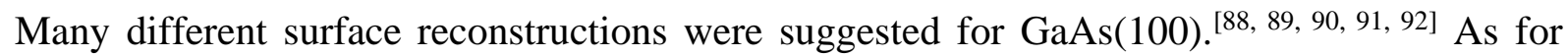
$\mathrm{GaP}(100)$ and $\mathrm{InP}(100)$ surfaces discussed above, spectral features of GaAs(100) surfaces were assigned to dimers on the surface ${ }^{[93,94]}$ and the spectra vary strongly in dependence on the rate of As coverage on the surface. ${ }^{[95,96]}$ Indeed, also calculations of the RAS lineshape for differently reconstructed $\mathrm{GaAs}(100)$ surfaces showed a strong dependence on the atomic structure of the surfaces. ${ }^{[97,98]}$ However, the entire surface geometry needs to be taken into account to yield an adequate agreement with experimental data. ${ }^{[98]}$ Excitonic effects were found negligible, ${ }^{[98]}$ while surface strain can contribute to the RAS signal. ${ }^{[99,}{ }^{100]}$ By 
comparison of RAS with DFT, ${ }^{[101]}$ the As-rich surface was assigned to $c(4 \times 4)$ and $(2 \times 4)$ phases, while the Ga-rich surface reconstructs $(4 \times 2)$. Schmidt et al. ${ }^{[11]}$ compared RA spectra obtained by DFT and in experiment, respectively. A mixed $\mathrm{Ga}-\mathrm{As}$ dimer structure is energetically most favorable for "extreme" Ga-rich conditions, ${ }^{[91]}$ and causes an RA spectrum similar to that experimentally observed for (nx6) surface structures. ${ }^{[101]}$ The $(2 \times 4)$ and $c(4 \times 4)$ reconstructed GaAs(100) surface exhibits surface states, which contribute to the spectral RAS features below the $E_{1}$ bulk critical point. ${ }^{[80,101,102,103]}$ The RA spectrum above $E_{1}$, in contrast, is assigned to surface modified bulk transitions. ${ }^{[102,104,105]}$

Fig. 10 displays color-coded RA spectra during annealing in $\mathrm{H}_{2}$ ambient. The development from the As-rich to the Ga-rich surface (via intermediate surface reconstructions) by desorption of As due to the increased temperature is clearly observable by the different RA spectra associated with the surface reconstructions. Here, the significant differences in the lineshapes enable to study the surface formation in situ with RA transients, which must be done with care in other cases since temperature induced spectral shifts occur as well. ${ }^{[40]}$ Exploiting the in situ RA spectra of GaAs as a measure of crystal quality, process parameters could be tuned to increase the efficiency with regard to minimized material consumption ${ }^{[106]}$ and precursor choice. ${ }^{[107]}$

Fermi-level pinning at the surface can be caused by impurity induced surface states and contributes to RAS via the linear electro-optic effect. ${ }^{[108,}{ }^{109]}$ Combined with precise knowledge about changes in the surface reconstruction on temperature and dopant adsorption,

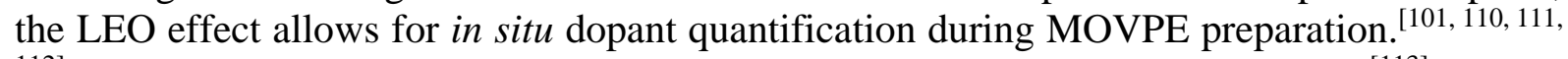
${ }^{112]}$ Different regimes for C-doping were studied via their surface reconstructions. ${ }^{[113]}$ Just as for InP and GaP, the temperature dependence of the dielectric function of $\mathrm{GaAs}^{[114]}$ was measured by in situ ellipsometry in MOVPE ambient. ${ }^{[115]}$

A notable exception of the merely optical in situ approaches are the first MOVPE in situ scanning tunneling microscopy (STM) images that were obtained at GaAs(100) surfaces at temperatures up to $650{ }^{\circ} \mathrm{C}$ and at atmospheric pressures. ${ }^{[16]}$ Even though the limited resolution is a drawback compared to STM at ambient or cryogenic temperatures, the in situ STM approach was also used to study quantum dot formation. ${ }^{[117,118]}$

\subsection{Multinary III/V compounds}

Ternary and quaternary III/V compound semiconductors are highly relevant in order to tune the band gap, lattice constant and optoelectronic properties as desired for the device of choice (cf. Fig.2). Multinary epilayers may be used as active parts of the device ${ }^{[119]}$ (such as InGaAsP-based absorbers), barrier layers to tune the band alignment or simply as transition layers, for instance, to bridge lattice mismatch between two materials by graded buffers. ${ }^{[120,76]}$ It often is useful to study constituents of multinary compounds individually. ${ }^{[121]}$ For multinary compounds, optical real time analysis can contribute to "control of thickness and stoichiometry during growth as well as monitoring the switching procedures during the growth of heterostructures." ${ }^{[122]}$ For example, the MOVPE growth of entire laser and multijunction solar cell (MJSC) structures can be monitored in situ with RAS. ${ }^{[123,124]}$ For multinary III/V compounds, the choice of a suitable growth regime is more important compared to the rather stable growth of binaries. Often, different growth conditions also relate to different surface structures, which can be observed with RAS. For InGaP, for example, bulk ordering was observed for P-rich growth conditions - which can be correlated with a characteristic RAS signal. ${ }^{[125,126]}$ 
A combined ex situ X-ray diffraction (XRD), ellipsometry and in situ RAS study revealed that the In content in $\operatorname{In}_{\mathrm{x}} \mathrm{Ga}_{1-\mathrm{x}} \mathrm{As}$ can be obtained in situ since the composition of the uppermost layers affects the measured optical anisotropy: ${ }^{[127]}$ As displayed in Fig. 11, starting from a $\mathrm{c}(4 \times 4)$ reconstructed $\mathrm{GaAs}(100)$ surface and its characteristic RA spectrum, the RAS lineshape changes towards that of $(1 \times 3)$ reconstructed $\operatorname{InAs}(100),{ }^{[128]}$ when the In content is increased. ${ }^{[127]}$ In the same work, monolayer oscillations during InGaAs growth on GaAs(100) were observed. ${ }^{[127]}$ Analysis of in situ RA spectra benefits from knowing the dielectric constants of the compound at growth temperature. ${ }^{[129]}$ Similar to InGaAs, the stoichiometric dependence of the lineshape of the RAS signal enables evaulation of the As/P content of GaAsP(100) surfaces in situ, as indicated in Fig. 12. ${ }^{[127,130]}$ The dependence of the RAS signal on the In and $\mathrm{P}$ content in InGaAs quantum wells (QWs) and strain compensating GaAsP layers was used also during MOVPE growth of laser diodes, and strain balancing studied in situ via the RAS signal of GaAs. ${ }^{[131]}$ For GaAsP QWs, the emission wavelength could be correlated to the amplitude of the RAS signal. ${ }^{[132]}$ Similar to GaAs, the impact of doping on the RAS signal has been studied for multinary III/V compounds, such as InGaAs, AlGaAs and AlGaInP. ${ }^{[58,132]}$ Layer thickness, monolayer oscillations and composition can be measured as well with in situ ellipsometry during MOVPE growth, as demonstrated for instance for InGaAs and AlGaAs. ${ }^{[133,134]}$ AlGaAs was also studied with RAS in detail both in MOVPE $^{[135]}$ and in UHV. ${ }^{[136]}$ Increasing complexity to quaternary compounds, composition and growth rate of InGaAsP and AlGaInP can be obtained combining in situ RAS and reflectance measurements during MOVPE growth. ${ }^{[137,138,139,140]}$

Broadening the range of compounds to dilute nitrides, it is worth to mention that also the RAS signal of GaAsN changes with the $\mathrm{N}$ content, ${ }^{[11,141]}$ and reflectance measurements have been applied to yield the composition. ${ }^{[11,142]} \mathrm{N}$ incorporation into $\mathrm{GaP}$ at diluted concentration (grown heteroepitaxially on $\mathrm{Si}$ with about $2 \% \mathrm{~N}$ to yield lattice-matching at room temperature) was found to cause an additional contribution to the RAS signal at the $\mathrm{E}_{1}$ interband transition of $\mathrm{GaP}$, while the principal features of the surface reconstructions typical for $\mathrm{GaP}(100)$ could be preserved — if excess $\mathrm{N}$ at the surface was avoided. ${ }^{[20]}$

Besides the optical techniques commonly applied in situ during MOVPE preparation, in situ $\mathrm{X}$-ray monitoring during InGaAs growth enabled determination of layer thickness and lattice mismatch as well as its temperature dependence. ${ }^{[143]}$

\subsection{Nanostructures}

A broad spectrum of microscopy techniques has also been applied for the in situ characterization of nanowires and nanostructures, ${ }^{[10]}$ but mostly in vacuum environment. In practical MOVPE ambient, in situ reflectance during nanowire growth paired with optical modeling enables to determine the nanowire dimensions as well as their growth rate. ${ }^{[44]}$ Interesting in situ approaches include in situ photoluminescence (PL), which was demonstrated to predict the emission wavelength of InGaN QW-based light emitting diodes (LEDs) already during growth. ${ }^{[145,146]}$ Ostwald ripening of InAs quantum dots on GaAs(100) could be observed with in situ STM, ${ }^{[17]}$ and a combined RAS / in situ STM study revealed the dependence of InGaAs quantum dot formation on different surface reconstructions. ${ }^{[118]}$ InGaAs quantum dot formation and island nucleation was studied also by in situellipsometry. ${ }^{[147]}$

\section{6. $\operatorname{Si}(100)$}


Regarding highly efficient tandem absorber structures for solar energy conversion, group IV substrates are often preferred over III/V substrates: Germanium, for example, is more suitable as substrate used in the industry, when manufacturing standard triple junction solar cells, than GaAs, since the bandgap energy is lower and substrate costs are considerably cheaper. III/Von-Si integration is considered to further reduce costs of solar cells, ${ }^{661,148,149,150,151,152]}$ increase efficiency ${ }^{[153]}$ and is also desired for microelectronics. ${ }^{[154]}$ In contrast to III/V crystal structures, both Si and Ge substrates do only have covalent bonds between two atoms of the identical kind and are thus nonpolar lattice structures. The step structure and atomic structure of $\mathrm{Ge}(100)$ and $\mathrm{Si}(100)$ surfaces therefore is highly important for subsequent low-defect heteroepitaxy of polar III/V semiconductors on nonpolar substrates: Steps of odd atomic height on the nonpolar substrate introduce antiphase disorder in subsequently grown, polar III/V epilayers. ${ }^{[155,156]}$ Since both $\mathrm{Si}(100)$ and $\mathrm{Ge}(100)$ are dimerized under standard MOVPE preparation conditions, it is useful to express the step structure via the dimer orientation at the associated terraces. An established nomenclature ${ }^{[157]}$ is that of A-type and B-type terraces $\left(\mathrm{T}_{\mathrm{A}}\right.$ and $\left.\mathrm{T}_{\mathrm{B}}\right)$ for $(1 \times 2)$ and $(2 \times 1)$ reconstructed surfaces, respectively, which is illustrated in Fig. 13. Due to the tetrahedral coordination in the diamond lattice, the dimer orientation rotates by $90^{\circ}$ on adjacent terraces separated by steps of mono- or odd-atomic height. Depending on the upper terrace, these are called $\mathrm{S}_{\mathrm{A}}$ and $\mathrm{S}_{\mathrm{B}}$ steps. Terraces separated by double-layer steps exhibit dimer rows in parallel to each other and are labelled $\mathrm{D}_{\mathrm{A}}$ and $\mathrm{D}_{\mathrm{B}}$. In the following, we will discuss single-domain $\mathrm{Si}(100)$ and $\mathrm{Ge}(100)$ surface preparation, which enables III/V growth free of antiphase disorder.

\subsubsection{Monohydride-terminated Si(100) surfaces}

\section{Surface termination}

Unreconstructed $\mathrm{Si}(100)$ surfaces exhibit two dangling bonds per $\mathrm{Si}$ atom because of the diamond crystal structure. To minimize the surface energy, buckled dimers form in UHV ${ }^{[158}$, $159,160]$ and also the step structure at $\mathrm{Si}(100)$ misoriented towards [011] is governed by

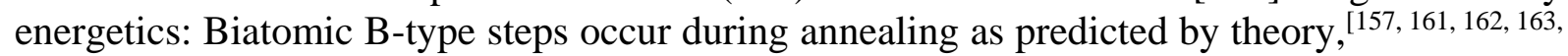
${ }^{164]}$ while biatomic A-type steps are energetically less favorable than D-type steps as well as single atomic steps. ${ }^{[157]}$ Their formation in UHV requires external forces. ${ }^{[165,166,167,168]}$ Dependent on the temperature and $\mathrm{H}$ chemical potential (corresponding to $\mathrm{H}$ supply), $\mathrm{Si}(100)$ surfaces, which are exposed to atomic hydrogen in UHV, form differently H-passivated reconstructions. ${ }^{[162,169,170]}$ Symmetric monohydride-terminated Si dimers with one $\mathrm{H}$ atom per $\mathrm{Si}$ atom form upon annealing in $\mathrm{H}_{2}$ ambient. ${ }^{[171,172,173,174]} \mathrm{H}_{2}$ process gas is also commonly used during MOVPE preparation. Indeed, attenuated total reflection (ATR) FTIR measurements on MOVPE-prepared $\mathrm{Si}(100)$ surfaces have identified monohydride $\mathrm{Si}-\mathrm{H}$ bonds at the surface ${ }^{[175,176]}$ via measurement of their coupled stretch modes, ${ }^{[177,178]}$ and tipinduced $\mathrm{H}$ desorption by STM has confirmed complete hydrogen coverage. ${ }^{[176]}$ In situ RAS during cooling from $1000{ }^{\circ} \mathrm{C}$ in $950 \mathrm{mbar} \mathrm{H}_{2}$ ambient verified that $\mathrm{Si}(100)$ is terminated by monohydrides at temperatures below $800{ }^{\circ} \mathrm{C} .{ }^{[179]}$ The dependence of the $\mathrm{H}$ coverage depends highly on MOVPE process temperature and reactor pressure. ${ }^{[179,180,181]}$

For such H-terminated $\mathrm{Si}(100)$ surfaces, energetics change drastically: Non-rebonded singlelayer steps at monohydride-terminated $\mathrm{Si}(100)$ are energetically favorable, ${ }^{[182,}$ 183] as confirmed by STM studies in UHV in presence of atomic $\mathrm{H}^{[184]}$ In contrast, however, experiments show that $\mathrm{S}_{\mathrm{A}}$ steps vanish in favor for biatomic steps during annealing in $\mathrm{H}_{2}$ ambient. ${ }^{[185]}$ Also early $\mathrm{H}_{2}$-based MOVPE experiments reported on antiphase domain (APD) free $\mathrm{GaP}$ growth on $\mathrm{Si}(100)$ with $2^{\circ}$ misorientation, ${ }^{[186]}$ which implies an unequal domain distribution at the Si surface. A prevailing majority domain with an unspecified type of step structure was also observed during MOVPE preparation of nominal Si(100) surfaces. ${ }^{[187]}$ Quantitatively, FTIR studies surprisingly revealed a preference for the A-type domain after 
slow cooling in $\mathrm{H}_{2},{ }^{[175]}$ which is considered the least favorable configuration in terms of energetics.

\section{Surface formation: Energetics vs. kinetics}

The results discussed above imply a non-negligible impact of kinetic processes during MOVPE preparation of the $\mathrm{Si}(100)$ surfaces, which may counteract energetic considerations. Due to the strong anisotropy of the dimerized surface reconstruction, RAS is ideally suited to study $\operatorname{Si}(100)$ surface formation. While in principle also dimer induced $\operatorname{strain}^{[188]}$ can contribute to RA spectra of $\mathrm{Si}(100),{ }^{[189,190]}$ the main bulk-like contribution is supposed to be induced by the anisotropic surface potential. ${ }^{[190,191]}$ Differently H-terminated $\mathrm{Si}(100)$ surfaces exhibit RA spectra with different characteristic lineshapes. ${ }^{[191]}$ They can thus be identified in situ. The fingerprint of the dimers at monohydride-terminated Si(100) surfaces (Fig. 14(a)) consists of two peaks (at the $\mathrm{E}_{1}$ and close to the $\mathrm{E}_{2}$ interband transitions, respectively) and a shoulder in between. ${ }^{[191,192,193]}$ This spectrum relates to the dimers at the terraces, which enables an in situ quantification of the domain ratio: ${ }^{[191,193]}$ By definition of the RAS signal (eq(1)), a $90^{\circ}$ rotation of the dimer axis flips the sign of the spectrum. Contributions of domains consisting of dimers with mutually perpendicular dimer orientation thus cancel via inherent integration over the spot size. The measured sign of the signal then corresponds to the majority dimer orientation and its intensity yields the domain imbalance when scaled to a single-domain reference. ${ }^{[68,191,193]}$

In Fig. 14(a), the black RA spectrum corresponds to a monohydride-terminated $\mathrm{Si}(100)$ surface with a B-type majority domain and an A:B imbalance of about 40:60. ${ }^{[191]}$ It was prepared in UHV with atomic H. ${ }^{[191]}$ In strong contrast, in situ RAS reveals that the energetically less favored A-type terraces form during annealing of $\operatorname{Si}(100)$ surfaces with $2^{\circ}$ misorientation towards [011] in 950 mbar $\mathrm{H}_{2}$ ambient (Fig. 14, green line) under certain annealing conditions ${ }^{[193]]}$ after thermal deoxidation. ${ }^{[194]}$ Despite sign and increased intensity (indicating a strong domain imbalance of $\mathrm{A}: \mathrm{B}$ of about 0.85 ), the lineshapes of the RA signals of monohydride-terminated $\mathrm{Si}(100)$ are almost identical (Fig. 14(a)), despite different magnitudes of misorientation. Step contributions to the signal ${ }^{[190]}$ can thus be neglected here. Consequently, time-resolved RA measurements enable the observation of terrace formation in situ and demonstrate that thermal treatment with temperatures in the range of $730-750{ }^{\circ} \mathrm{C}$ is required for the development of such an A-type surface (Fig. 14(b)). ${ }^{[193]}$ The important point is, that this surface formation is dominated by kinetic processes rather than energetics. Strong interaction with the $\mathrm{H}_{2}$ process gas ${ }^{[179]}$ creates $\mathrm{Si}$ vacancies at the surface, which diffuse preferably along dimer rows on the anisotropic terraces. ${ }^{[195]}$ If the terrace width is small compared to the vacancy diffusion length, $\mathrm{T}_{\mathrm{B}}$ terraces retreat via vacancy annihilation at the end of the dimer rows, ${ }^{[165,168,195]}$ as shown in Fig. 14(c). At larger terraces (lower offcut), single vacancies may coalesce to vacancy islands, ${ }^{[165,195]}$ resulting in a Si layer-by-layer removal. ${ }^{[196]}$ This can be observed in situ at $\mathrm{Si}(100) 0.1^{\circ}$ as oscillations in transient $\mathrm{RA}$ measurements due to the dimer rotation at every subjacent terraces (Fig. 15). ${ }^{[196]}$ An activation energy of $2.8 \pm 0.2 \mathrm{eV}$ was derived via in situ RAS and attributed to $\mathrm{SiH}_{\mathrm{x}}$ formation. ${ }^{[196,197]}$ Since residuals at the step edges may remain, continued Si removal leads to roughened surfaces with frayed step edges. ${ }^{[196]}$ In contrast to $\mathrm{Si}(100) 2^{\circ}$, annealing at about $730{ }^{\circ} \mathrm{C}$ in high $\mathrm{H}_{2}$ pressure is not beneficial for almost nominal $\mathrm{Si}(100)$. The preparation route for $\mathrm{Si}(100) 2^{\circ}$ is rather robust in a larger window of parameter variation and yields almost single-domain surfaces suitable for III/V nucleation. ${ }^{[198]}$ It is important to realize that surface processes at $\mathrm{Si}(100)$ in hydrogen ambient also and strongly depend on the misorientation: The formation of B-type Si surfaces is more likely on vicinal surfaces, where also a step contributions can occur in the RA spectra. ${ }^{[181]}$ 


\subsubsection{Arsenic-modified Si surfaces}

Despite the fact that arsenic (As) is present as residual in many application-relevant MOVPE reactors anyway, As-modification of $\mathrm{Si}(100)$ surfaces is of high interest for at least two reasons: (i) Compared to thermal deoxidation, ${ }^{[199]} \mathrm{HF}$ dipping plus processing in an As ambient can significantly reduce the thermal budget of the MOVPE Si process. ${ }^{[200,201]}$ (ii) As in-diffusion into $\mathrm{Si}(100)$ is promising with regard to in situ preparation of $\mathrm{Si}$ p-n junctions, ${ }^{[202,}$ ${ }^{203]}$ while an As-termination at the same time may prohibit Si in-diffusion into subsequently grown III/V epilayers. ${ }^{[204]}$ Early UHV studies revealed that different process routes determine whether As either adsorbs additive on $\mathrm{Si}(100)$ (on top) or replaces $\mathrm{Si}$ atoms in the topmost layer(s), which enabled the adjustment of the sublattice orientation of subsequently grown GaAs epilayers. ${ }^{[205]}$ In situ RA spectra of these predominantly $(1 \times 2)$ and $(2 \times 1)$ reconstructed, As-modified $\mathrm{Si}(100)$ surfaces were assigned to optical transitions involving states induced by the As dimers. ${ }^{[206]}$ This was questioned by MOVPE studies showing similar signals also on two-domain surfaces. ${ }^{[200,207]}$ Recent results demonstrated MOVPE preparation of singledomain As-modified Si(100) surfaces $^{[208,209]}$ and suggest different microscopic origins for the two main anisotropic RAS contributions. ${ }^{[208]}$

X-ray photoelectron spectroscopy (XPS) results imply As intermixing in near-surface layers. ${ }^{[208]}$ Similar to As-free systems, the RAS signal can be applied in situ to determine adequate temperature and As-pressure regimes for surface ordering, which is useful to avoid roughening or line defects that may occur. ${ }^{[200,208,209,210]}$ For the MOVPE-prepared surface, it is still under debate, whether the surface is terminated by As or monohydride Si dimers on top of an As interlayer, a mix of both or possibly $\mathrm{Si}-\mathrm{As}$ heterodimers. ${ }^{[208]}$ An increased intensity of the spectral contribution at the $\mathrm{E}_{1}$ interband transition of $\mathrm{Si}$ in comparison to the UHV prepared surface, however, indicates that monohydride-termination dimers may in part be present. To resolve this question, in situ studies in dependence of the amount of As being offered in combination with FTIR are currently ongoing in our labs.

\subsection{Ge(100)}

Similar to $\mathrm{Si}(100)$, the influence of hydrogen was studied in situ during $\mathrm{Ge}(100)$ surface formation. In contrast to $\mathrm{Si}$ substrates in the CMOS technology, vicinal Ge surfaces are mostly applied in MJSC solar cell fabrication due to their tendency to form double-layer steps and due to the easier adjustment of appropriate doping concentrations. To reduce the surface energy, clean Ge surfaces are reconstructing via the formation of dimers with $(2 \times 1),(2 \times 2)$, or $\mathrm{c}(4 \mathrm{x} 2)$ symmetry. ${ }^{[211]}$ Monohydride-termination along with a $(2 \times 1)$ reconstruction occurs during exposure to atomic $\mathrm{H}$ in UHV. ${ }^{[178]}$ Assisted by benchmarking measurements to UHVbased surface science techniques, different RAS fingerprints were established for B-type monohydride-terminated and clean Ge(100) surfaces (cf. Fig. 16). ${ }^{[212,213,214]}$ This enabled in situ studies on the preparation of clean and monohydride terminated $\mathrm{Ge}(100)$ surfaces and the $\mathrm{H}$ desorption kinetics in MOVPE ambient, respectively. ${ }^{[215]}$ From DFT calculations and comparison to experimental results, ${ }^{[216]}$ however, the microscopic origin of the RA spectra could not yet be clarified due to lack of low temperature RAS data.

Thermal annealing in $\mathrm{H}_{2}$ ambient enables complete removal of oxygen and other contaminations from oxidized "epiready" wafers. ${ }^{[214]}$ Analogous to $\mathrm{Si}(100), \mathrm{Ge}(100)$ surfaces are monohydride terminated after processing in $\mathrm{H}_{2}$ ambient, as verified by FTIR measurements ${ }^{[214]}$ (cf. Fig. 17). The hydrogen coverage of the Ge surface during processing in $\mathrm{H}_{2}$ ambient represents a dynamic balance of hydrogen adsorption and desorption events depending on the process parameters such as temperature and hydrogen pressure. Accordingly, $\mathrm{Ge}(100)$ is $\mathrm{H}$-free in $\mathrm{H}_{2}$ process gas ambient at a $\mathrm{H}_{2}$ pressure of $100 \mathrm{mbar}$ for temperatures above $370{ }^{\circ} \mathrm{C}$, and thus corresponding process conditions are typically used for 
III/V nucleation $\left(420-750{ }^{\circ} \mathrm{C}, 50-100 \mathrm{mbar} \mathrm{H}_{2}\right.$ pressure $) .{ }^{[217,218,219,220,221]}$ In contrast to $\mathrm{Si}(100)$, there is no indication for etching processes induced by the $\mathrm{H}_{2}$ interaction. The vicinal Ge(100) substrates exhibited a clear (2×1)/B-type majority domain and mainly Dв double layer steps, respectively (see Fig. 16). These findings serve as a reference point to study the influence of As and P on the surface structure of vicinal Ge(100) substrates.

With regard to III/V nucleation, ${ }^{[222,223,224,225]}$ it is important to understand the impact of group V elements on the atomic order of the Ge(100) surface, since III/V-on-Si or III/V-onGe heteroepitaxy typically starts with exposure to group V precursors. Exposure of $\mathrm{Ge}(100)$ to As strongly affects the As dimer orientation, the height of steps, and the atomic configuration at the step edges, ${ }^{[226,227,228]}$ which in turn affects the subsequent $\mathrm{GaAs}$ nucleation. ${ }^{[222,225,224,229]}$ Process temperature, source, and partial pressure of arsenic are key parameters for the $\mathrm{Ge}(100)$ :As surface preparation. ${ }^{[26,227]}$ In particular, annealing in tertiarybutylarsine (TBAs) or $\mathrm{AsH}_{3}$ at $650{ }^{\circ} \mathrm{C}$ resulted in a $\mathrm{Ge}(100)$ ): As surface with prevalence for As dimers oriented parallel to the step edges ( $\mathrm{Ge}(100)$ :AsB), while annealing at the same temperature in the presence of background As4 from residual GaAs reactor coatings led to a surface with As dimers oriented perpendicular to the step edges (Ge(100):AsA). STM measurements (see Fig. 16) revealed distinct differences in the step structure of $\mathrm{Ge}(100)$ :AsB and $\mathrm{Ge}(100)$ :AsA surfaces, in particular, formation of multiple layer steps due to step bunching after annealing in TBAs or $\mathrm{AsH}_{3}$, which is known to etch the $\mathrm{Ge}(100)$ surface, and mainly quadruple-layer steps separating A-type terraces after annealing in background As4, respectively. ${ }^{[226,230]}$

Characteristic RA spectra of vicinal Ge(100):As surfaces were established for MOVPE preparation applying tertiarybutylarsine (TBAs) and background As4 as As sources (cf. Fig. 16) and benchmarking to results from surface analysis by LEED, XPS, and STM. ${ }^{[230]}$ The corresponding RA spectra contain contributions from both steps and terraces and enable in situ control over the domain formation. ${ }^{[230]}$ While the rotation of the major As dimer orientation depending on the process conditions causes a flip of the characteristic features of the $\mathrm{Ge}(100)$ :As RAS signal, additional differences in the RA spectra were assigned to the different step structure. ${ }^{[230]}$ Temperatures in the range of $670{ }^{\circ} \mathrm{C}$ were found to be crucial for the formation of the surface structure. ${ }^{[230]}$ Transient RAS measurements confirmed fast flipping of the major As dimer orientation on $\mathrm{Ge}(100)$ after changing the source of As (switching from As from precursor to background As4). Similar to the observations on the $\mathrm{Si}(100)$ surface in $\mathrm{H}_{2}$ ambient, surface energetics as well as kinetics compete during step and domain formation induced by thermal treatment and the interaction between the $\mathrm{AsH}_{3}$ or As4 and the Ge(100) surface. The in situ RAS measurements enable direct characterization of the highly sensitive domain formation under different reactor conditions.

Nucleation of GaInP on Ge(100) in MOVPE environment is usually obtained by $\mathrm{P}$ termination of the $\mathrm{Ge}(100)$ surface prior to heteroepitaxy. ${ }^{[217,}{ }^{231]}$ Phosphine $\left(\mathrm{PH}_{3}\right)$ and tertiarybutylphosphine (TBP) are the two main $\mathrm{P}$ precursors used in MOVPE systems. Annealing under $\mathrm{PH}_{3}$ at $300-450{ }^{\circ} \mathrm{C}$ in low pressure CVD systems leads to $\mathrm{P}$ termination of $\mathrm{Ge}(100)$ surfaces by one atomic layer. ${ }^{[232]}$ LEED analysis of a P-terminated Ge(100) surface with $2^{\circ}$ offcut prepared by $\mathrm{PH}_{3}$ exposure in MOVPE ambient shows a (9x2) surface reconstruction. ${ }^{[231]}$ In contrast to $\mathrm{AsH}_{3}, \mathrm{PH}_{3}$ does not etch the Ge surface. ${ }^{[[233]}$ TBP annealing results in a rather disordered P-terminated surface covered by about $1.5 \mathrm{ML}$ of $\mathrm{P}$ and carbon contamination. The presence of carbon is attributed to byproducts of the TBP pyrolysis, since the $\mathrm{Ge}(100)$ surfaces are carbon free after $\mathrm{H}_{2}$ annealing. The vicinal $\mathrm{Ge}(100)$ surface annealed in TBP exhibits a characteristic RA spectrum, which can be assigned to the $\mathrm{P}$ termination of the surface. In situ RAS studies showed that the P termination is less stable during annealing 
in $\mathrm{H}_{2}$ ambient than the As termination: $\mathrm{P}$ desorption takes place at temperatures around $430{ }^{\circ} \mathrm{C}$ as indicated by a transition from the RAS signal of the $\mathrm{P}$ terminated surface to the signal of the clean surface (see Fig. 16).

Barrigon et al. ${ }^{[217]}$ found that the morphology of InGaP nucleation layers on Ge correlates to a specific RAS feature, which enables optimization the InGaP nucleation route via transient in situ RA measurements.

\section{Internal interfaces of epitaxial heterostructures}

The understanding of the atomic structure of the surfaces of III/ $\mathrm{V}$ compounds is important particularly during switching processes and heterointerface formation. ${ }^{[58,130,234]}$ There are different approaches to study the interface formation, of which we will choose three in the following:

(i) One can focus on the atomic structure of the substrate layer prior growth of the overlayer and analyze the impact on the properties of that layer. This will be exemplified for preparation of a tunnel junction in section 4.1 and for III/V-on-Si heterointerfaces in section 4.2.

(ii) If the top epilayers are sufficiently transparent, buried interfaces can be analyzed in situ with optical techniques. ${ }^{[235]}$ Regarding RAS, a buried heterointerface may contribute in two ways:

a. The interface itself may be optically anisotropic, for instance due to strain, bonds along preferential directions, or bulk-termination effects similar to surfaces. Such interface anisotropies will be discussed for $\mathrm{GaP} / \mathrm{Si}(100)$ in section 4.2.3.

b. The bare presence of the interface causes thickness-dependent Fabry-Pérot-like interference, which enters the RAS signal due to the normalization with the mean reflection. This can be exploited to obtain growth rates or dielectric constants, just as for reflectance measurements. If interference is considered adequately, antiphase disorder may be quantified from in situ RA spectra, as will be discussed in section 4.2.4.

(iii) Spectra or transients can be measured continuously during nucleation and interface formation, respectively. Transient reflectance measurements, which are typically used to measure growth rates and surface roughness, can also be applied to determine the interfacial roughness. ${ }^{[235]}$ Transient RAS measurements were applied to study $\mathrm{GaP}$ nucleation on $\mathrm{Si}^{[236,237]}$ and a recent example will be discussed in section 4.2.3

Due to the interference effects, care must be taken when analyzing RA spectra of heterostructures with regard to surface reconstructions. In simple heterostructures, interference can in principle be accounted for with an empirical approach: ${ }^{[68]}$ If the reflectance signal of a reference surface of the overlayer material is available, one can calculate a socalled relative reflectance spectrum (reflectance of the heterostructure divided by that of the reference). Multiplication of the RAS signal with the relative reflectance signal corrects interference - under the assumption of isotropic interfaces (and identical apparatus function for the two reflectance measurements). ${ }^{[68]}$ 
Optical modelling, in contrast, enables the extraction of both the surface, interference and "real" interface contributions from the RA spectra (cf. Fig. 18). Assuming the anisotropies to be small, a Taylor expansion of the RAS signal yields an expression for the superposition of the surface, interface and (overlayer) bulk anisotropies with prefactors that only depend on optical constants and the overlayer thickness. ${ }^{[238]}$ Assuming that the actual anisotropies do not depend on the layer thickness, one yields a system of equation that can be solved for the anisotropic contributions. This three-layer approach was first demonstrated for $\mathrm{ZnSe} / \mathrm{GaAs}(100)^{[239,240]}$ and $\mathrm{SiO}_{2} / \mathrm{Si}^{\left[{ }^{[238,241]}\right.}$ Surface and interface anisotropies have also been separated for AlAs/GaAs(100). ${ }^{[99]}$ The three-layer model was extended to a five-layer model to study the interface between $\mathrm{AlAs} / \mathrm{GaAs}(100),{ }^{[242]}$ and it was further modified to enable also fitting for the APD content of III/V-on-Si(100) heterostructures. ${ }^{[243]}$

\subsection{III/V heterostructures in multi-junction cells}

Strain and strain relaxation can be monitored in situ via the wafer curvature / bowing, which is particularly important for mismatched III/V heterostructures. ${ }^{[244,245]}$ Interdiffusion can be studied with in situ ellipsometry to find optimized growth conditions for abrupt interfaces. ${ }^{[246]}$ Interference-caused oscillations in RAS and reflectance measurements can be fitted to obtain the growth rate of the overlayers, and the intensity can be used to indicate the beginning of surface roughening. ${ }^{[247]}$ Here, however, we will focus on in situ RAS studies related to the formation of heteroepitaxial junctions, which are applied in high-performance multi junction cells.

Inherent conversion losses in light absorbers originate from thermalization of electron-hole pairs generated by photons with energy larger than the band gap and from radiation of photons with energy smaller than the band gap of the semiconducting absorber material. These losses can be reduced, if multiple single junction cells with different band gaps are connected in series in monolithical stacks comprising a plurality of cells connected in series and deposited on substrates such as $\mathrm{Si}, \mathrm{Ge}, \mathrm{GaAs}$, or InP. The cells are selected with appropriate band gaps to efficiently generate photovoltage from a larger portion of the solar spectrum and to achieve current-matching in monolithic stacks. To facilitate photocurrent flow in a multi junction stack, tunnel junctions of low-resistivity materials are inserted between each adjacent semiconductor cell, and charge separating heterojunctions are introduced next to the contact layers to extract charge carriers selectively. ${ }^{[248]}$ The theoretical upper limit of the conversion efficiency for a multiple solar cell configuration of infinitely many subcells with different band gaps adds up to about $86 \% .^{[249]}$ The formation of abrupt interfaces is of major importance at several positions in the layer structure of such multi junction solar cells. In particular, the performance of a tunnel junction critically depends on its spatial extension, i.e., the sharpness of its interfaces. Interfacial sharpness is also crucial for interfaces to charge separating contacts (so-called window- and BSF-layers), which are directing the different charge carriers, electrons and holes, appropriately.

A low band gap tandem (two-junction) solar cell was suggested almost a decade ago ${ }^{\text {[27, 28, 29] }}$ as part of a four junction solar cell: This two-junction tandem as lower part of the four junction solar cell consists of an InGaAs bottom cell and InGaAsP top cell, both latticematched to InP, and can be optimized for absorption in the solar infrared spectrum underneath a well-established GaAs/InGaP top tandem. Such a low-bandgap tandem is part of the current record efficiency solar cell, ${ }^{[12,13,30,31]}$ and its structure is shown schematically in Fig. 4. The complete stack of the wafer-bonded $\mathrm{InGaAs} / \mathrm{InGaAsP} / /(\mathrm{In}) \mathrm{GaAs} / \mathrm{InGaP}$ four junction solar cell, comprises more than twenty, partially delicate interfaces. Different leading groups in the 
high-performance photovoltaics field currently work on improving that type of multi junction solar cell for its development towards $50 \%$ conversion efficiencies.

The electronic structure across relevant interfaces in these cells highly depends on the atomic order and the sharpness of the heterointerface, which in turn requires an adequate preparation sequence. The interface formation does not only depend on the two materials to be combined but also on the sequence during growth. The transition from GaAs to InAs occurs quickly, for instance, while In segregation was suggested to extend the interface from InAs to GaAs over several monolayers. ${ }^{[120]}$ When switching from As- to P- containing compositions, the carryover of As into the subsequent P-containing layers is also well-known, such as for InGaAs/InGaP ${ }^{[250]}$ or InGaAs/InP ${ }^{[251,252,253,254]}$ and is of particular interest for single interlayers of $\mathrm{GaP}$ in GaAs. ${ }^{[255]}$ In the tandem structure optimized for the infrared (Fig. 4), bandgaps of $1.03 \mathrm{eV}$ (InGaAsP) and $0.73 \mathrm{eV}$ (InGaAs) were utilized for the top and bottom subcell, respectively. It includes several critical interfaces (InP / InGaAs, InGaAs / InP, InGaAs / GaAsSb, GaAsSb / InP , InP / InGaAsP, GaAsP / InP) and, in particular, a specific interband tunnel junction including thin and highly doped layers of n-type InGaAs and p-type GaAsSb was used to connect the subcells.

The dependence of the RA spectrum on the InGaAs stoichiometry has been discussed above (section 2.4). On the lattice constant of InP, however, the In content is significantly larger. Fig. 19 displays RA spectra of $\operatorname{In}_{0.53} \mathrm{Ga} 0.47$ As grown on InP by MOVPE. ${ }^{[27]}$ The lineshape was found to depend significantly upon annealing conditions, which can (similar to GaAs) be related to different surface reconstructions: By benchmarking to LEED, one As-rich $(4 \times 3)$ reconstructed surface, a group III-rich $(2 \times 4)$ reconstructed surface and a group III-rich $(4 \times 2)$ reconstructed surface was identified. ${ }^{[21]}$ Transient RA measurements enabled identification of suitable process condition regimes for their preparation. ${ }^{[256]}$ The transition from As-rich to group III-rich surfaces, for instance, highly depends on the temperature at purging sequences. ${ }^{[256]}$ For the $(4 \times 2)$-reconstructed surface, surface states were also studied with energy-dependent UPS. ${ }^{[257]}$

The surface reconstruction of InGaAs(100) impacts the sharpness of the tunnel junction: Fig. 19(b) shows RA spectra of $2 \mathrm{~nm}$ thin GaAsSb layers grown on the three differently reconstructed InGaAs surfaces displayed in Fig. 18(a). ${ }^{[27]}$ Their lineshape varies strongly in dependence of the InGaAs/InP template surface, even though all three GaAsSb(100) surfaces exhibit an As-rich c $(4 \times 4)$ surface reconstruction. XPS studies, however, revealed that the $\mathrm{Sb}$ content of the GaAsSb is too low when grown on As-rich, $(4 \times 3)$ reconstructed $\mathrm{InGaAs}^{[27]}$ which may affect the RAS signal. ${ }^{[258,259]}$ It was shown that sharper tunnel junctions can be prepared on the group-III rich $\operatorname{InGaAs}(100)$ surface, ${ }^{[27]}$ which increases the cell performance. ${ }^{[28]}$

For the same tandem structure, the impact of switching sequences on the interface recombination at the InGaAs / InP interface is crucial: The impact of different precursor switching sequences on the minority charge carrier life times in the corresponding semiconducting layers has been correlated ex situ with time-resolved photoluminescence (TRPL) measurements of InP / InGaAs / InP double heterostructures. ${ }^{[29,254]}$ For group-III-rich preparation, enhanced lateral homogeneity is achieved, which yields higher lifetimes compared to group-V-rich preparation. ${ }^{[254]}$

\subsection{III/V/Si(100) heterointerfaces}


It is highly instructive to consider the formation of the III/ $/ \mathrm{Si}(100)$ heterointerface as a three-step process, where each step has to be controlled precisely to yield well-ordered interface structures. First, the $\mathrm{Si}(100)$ surface has to be prepared such that it is mostly singledomain in order to avoid antiphase disorder already within the very first III/V epilayers (see section 3). Second, during the actual nucleation phase, $\mathrm{V}-\mathrm{Si}$ and/or III-Si bonds form within a nucleation layer of a thickness in the order of monolayers. In this step, it is not trivial to discriminate between the impact of precursor switching sequences and that of the residuals being present in practical growth ambient. Comparison of in situ signals obtained during and after $\mathrm{Si}$ surface preparation with clean reference signals therefore is highly valuable to determine when phase two actually begins. ${ }^{[260]}$ Third, a "thicker" nucleation buffer is grown, which acts as virtual substrate layer for further integration of other III/V compounds, whose choice depends on the device of interest. Ideally, this buffer layer should exhibit defect densities as low as possible and a surface reconstruction suitable for subsequent epitaxy (see section 2).

One of the most crucial challenges in III/V/Si(100) nucleation is rooted in the different crystal symmetries of Si (diamond) and III/V compounds (mostly zincblende), which is often referred to as "polar-on-nonpolar heteroepitaxy". ${ }^{[155]}$ The choice of GaP as nucleating compound enables separation of the polarity issue from the other challenges, such as lattice-mismatch and diverging thermal expansion coefficients. $\mathrm{GaP}$ is an adequate candidate for the virtual substrate since it can be grown pseudomorphically up to several tens of nanometers. For optical applications, also the transparency of $\mathrm{GaP}$ compared to other III/V compounds can be advantageous.

\subsubsection{The significance of a two-temperature GaP-on-Si nucleation process}

It is important to distinguish between the actual GaP nucleation phase (step two in the list above) and GaP buffer growth (step 3): Low-temperature migration enhanced epitaxy (MEE) in MBE promotes two-dimensional GaP nucleation on vicinal Si(100) substrates. ${ }^{[261,262]}$ Also already in early MOVPE studies, ${ }^{[186]}$ a two-step growth process was found to suppress defects in the GaP layer. ${ }^{[186]}$ Low temperature nucleation is beneficial to reduce interface roughening, ${ }^{[263,264,265]}$ which was observed, e.g., by in situ ellipsometry ${ }^{[264]}$ for nucleation at $600{ }^{\circ} \mathrm{C}$. Detailed in situ studies — combining RAS, p-polarized reflectance spectroscopy (PRS) and laser light scattering during pulsed $\mathrm{GaP}$ nucleation by chemical beam epitaxy ${ }^{[237,266]}$ $(\mathrm{CBE})$ as well as polarometry ${ }^{[267]}$ in MOVPE - revealed that TBP reacts immediately upon adsorption on the growth surface, ${ }^{[237]}$ and that the amount of Ga must be precisely balanced to minimize surface roughening, 3D nucleation and Ga droplet formation. ${ }^{[237,266,267]} 3 \mathrm{D}$ nucleation is also reduced when applying high V:III ratios. ${ }^{[268,}{ }^{269} 270^{]}$More recently, GaP nucleation by MOVPE was studied in great detail ex situ with transmission electron microscopy (TEM): A pulsed nucleation sequence with alternating TBP and TEGa pulses at about $400{ }^{\circ} \mathrm{C}$ in combination with buffer layer growth above $570{ }^{\circ} \mathrm{C}$ was established. ${ }^{[220]}$ As a result, virtual $\mathrm{GaP} / \mathrm{Si}$ substrates free of islands, twin defects and stacking faults can be achieved as long as Ga droplet formation can be avoided. ${ }^{[220,270]}$

\subsubsection{Silicon preparation in presence of GaP residuals}

Specific preparation routes for single-domain $\mathrm{Si}(100)$ surfaces require balancing of energetic and kinetic driving forces which govern the step and terrace formation. In "clean" hydrogen ambient (free of III/V residuals, see section 3), surface temperature and $\mathrm{H}_{2}$ pressure have to be controlled precisely in dependence of the step density of the Si surface. ${ }^{[181]}$ Outgassing of III/V residuals during Si surface preparation adds further complexity and in situ control becomes even more important. In situ mass spectrometry during annealing of the MOVPE reactor at $1000{ }^{\circ} \mathrm{C} / 950 \mathrm{mbar} \mathrm{H}_{2}$ after a standard $\mathrm{GaP} / \mathrm{Si}$ process revealed that $\mathrm{P}-$ and Ga- 
related species can be detected even after 30min. ${ }^{[260]}$ How much this outgassing affects Si preparation also depends on the actual Si processing route: the desorption from reactor walls and from the susceptor is increased at higher temperatures. This is an issue particularly for the preparation route considered ideal for nominal $\mathrm{Si}(100)$ surfaces: A quick reduction of $\mathrm{H}_{2}$ pressure at elevated temperatures is required to yield predominantly (1x2) reconstructed $\mathrm{Si}(100)$ surfaces, which increases the rate of desorbing species at a process stage where the $\mathrm{H}$ stabilization is not yet stable. ${ }^{[179,196,260]}$ The RA spectra of the Si surface still show the signature of the $\mathrm{Si}$ dimers at the $\mathrm{Si} \mathrm{E}_{1}$ interband transition but also modifications at lower and higher binding energies, which might be related to Ga species being adsorbed. ${ }^{[271]}$ Regarding GaP growth on such "pre-nucleated" surfaces, antiphase disorder was increased which would be in line with both $\mathrm{Si}-\mathrm{P}$ and $\mathrm{Si}-\mathrm{Ga}$ bond domains being present at one terrace (see next section). ${ }^{[260]}$ If the amount of background $\mathrm{GaP}$ was further increased, no characteristic Sirelated RAS signatures could be obtained anymore. Similarly, the $(2 \times 1)$ reconstruction of $2^{\circ}$ misoriented $\mathrm{Si}(100)$ could only be observed in "clean" reactor ambient. ${ }^{[198]}$ The preparation route for $2^{\circ}$ misoriented $\mathrm{Si}(100)-(1 \times 2)$, in contrast, bases on annealing at high $\mathrm{H}_{2}$ pressure, which was found less critical and enabled more stable processing. ${ }^{[198,260]}$ Based on this advanced Si surface preparation ${ }^{[193]}$ and the low-temperature pulsed GaP nucleation, ${ }^{[220]}$ in situ studies of single-domain GaP nucleation and the interface structure became feasible, as will be discussed in the following.

\subsubsection{Single-domain GaP nucleation and interface structure}

In situ RAS was applied in three different ways to study the GaP/Si interface: (i) RA spectra obtained directly prior GaP nucleation and after GaP growth can be correlated ${ }^{[198]}$ to indirectly conclude on the structure of the buried interface based on the assumption of an abrupt interface; (ii) surface and interface contributions to the final GaP/Si RA spectrum can be separated by optical modeling ${ }^{[243]}$ in analogy to what was discussed for III/V heterostructures above; (iii) time-resolved RA yields real in situ information on the nucleation process. ${ }^{[272]}$ While the optical modeling approach enables automated fitting for the APD content at the $\mathrm{GaP} / \mathrm{Si}(100)$ surface, ${ }^{[243]}$ the interpretation regarding the interfacial structures within all three approaches largely benefits from single-domain $\mathrm{Si}(100)$ substrates.

Approach (i) requires knowledge of the domain structure of the $\mathrm{Si}(100)$ surface prior nucleation of the GaP sublattice as input. Assuming that the Si dimers break during nucleation, but that the $\mathrm{Si}$ atoms retain their lattice sites (i.e., an abrupt interface), one can conclude whether $\mathrm{Si}-\mathrm{P}$ or $\mathrm{Si}-\mathrm{Ga}$ bonds prevail at the interface. LEED patterns of a reference $\mathrm{Si}$ substrate and converged electron beam diffraction (CBED) TEM of final GaP/Si structures were first used, ${ }^{[273]}$ but both information can be obtained more directly with in situ RASwhich is particularly important for the correct description of the $\mathrm{Si}(100)$ surface ${ }^{[198]}$ RAS studies on $\mathrm{Si}(100)$ substrates with $2^{\circ}$ misorientation towards [011] — whose preparation is less sensitive to residuals - revealed that the sublattice orientation of the GaP epilayer can be inverted by flipping the majority dimer orientation of the Si substrate from $(1 \times 2)$ to $(2 \times 1) .{ }^{[198]}$ In both cases, this can be explained by $\mathrm{Si}-\mathrm{P}$ bonds within the abrupt interface model. ${ }^{[198]}$ Also $a b$ initio DFT calculations predict that abrupt $\mathrm{Si}-\mathrm{P}$ interfaces are energetically more favorable than abrupt $\mathrm{Si}$ - Ga interfaces over large ranges of chemical potential ${ }^{[198,274,275]}$ and that TBP adsorption creates a "strong" $\mathrm{Si}-\mathrm{P}$ bond. ${ }^{[276]}$ Nevertheless, charge compensation at the buried interface requires interfacial intermixing due to the partial charges of $\mathrm{Si}-\mathrm{P}$ and $\mathrm{Si}-\mathrm{Ga}$ bonds, respectively. ${ }^{[277]}$ Such compensated interfaces exhibit even lower formation energies than abrupt interfaces, independently on the chemical potential during nucleation. ${ }^{[198,}$ ${ }^{275]}$ Approach (i) cannot directly conclude on the atomic structure. Studies on almost exactly 
oriented $\mathrm{Si}(100)$, however, enabled to invert the GaP sublattice orientation also in dependence on the amount of Ga being available during nucleation. ${ }^{[198,260]}$ Since RAS evidenced that the Si dimers prior nucleation were aligned identically, this dependence on the chemical potential was interpreted as transition from $\mathrm{Si}-\mathrm{P}$ to $\mathrm{Si}-\mathrm{Ga}$ bonds depending on the chemical potential, which was predicted by DFT only for abrupt interfaces.

Approach (ii) revealed that the buried $\mathrm{GaP} / \mathrm{Si}(100)$ heterointerface exhibits a characteristic optical anisotropy. ${ }^{243,272]}$ These findings also verify that the lineshape of the final $\mathrm{GaP} / \mathrm{Si}(100)$ spectra is caused by internal reflection ${ }^{[68]}$ and enable an advanced in situ quantification of antiphase disorder. ${ }^{[243]}$ The presence of an interfacial anisotropy hints to a rather well-ordered interface, but it cannot yet be concluded whether this is caused by interfacial bonds, which are aligned along one direction in projection on the (100) terrace plane, ${ }^{[242]}$ or, for example, by strain in the surrounding $\mathrm{Si}$ or $\mathrm{GaP}$ matrix close to the interface ${ }^{[275,278,279]}$ or by truncation of the bulk. ${ }^{[280,281]}$

Most recently, approach (iii) revealed that $\mathrm{GaP} / \mathrm{Si}(100)$ heterointerface forms already during the first (TBP, TEGa) pulse pairs at low temperature in rather P-rich conditions. ${ }^{[272]}$ For the following discussion see Fig. 20. By measuring both transient RA during pulsed GaP nucleation and RA spectra after five and ten pulse pairs, respectively, it was shown that the Si-related RA signal vanishes with the first TBP pulse during GaP nucleation. A characteristic optical anisotropy evolves during further pulsed nucleation. This contribution to the RAS signal remains during further pulsing and annealing in TBP, when contribution associated to the $\mathrm{GaP}(100)$ surface starts to superimpose. The lineshape of that nucleation-related signal exhibits significant similarity when compared to the interface-related anisotropy, which was deduced from thicker GaP/Si samples (cf. approach (ii)). This indicates that the interface which forms during pulsed nucleation does not significantly change upon further layer growth. In reference to earlier work, ${ }^{[237,236]}$ pulse-related modulations in the RA transient during nucleation were assigned to a periodically consumed surface reaction layer. These findings are in line with XPS results, which imply that the interface forms within the first three pulse pairs and that every subsequent pulse pair adds a GaP bilayer. ${ }^{[272]}$ The in situ RA spectra indicate that a $\mathrm{GaP}$ surface with the characteristic surface reconstruction related to buckled $\mathrm{P}$ dimers forms already after 10 alternating (TBP,TEGa) pulses at low temperature and subsequent short annealing in TBP at $600{ }^{\circ} \mathrm{C}$. Benchmarking to LEED revealed that these surfaces indeed exhibit the well-known $(2 \times 2) / \mathrm{c}(4 \times 2)$ surface reconstruction-and that they are single-domain, which is highly relevant for their application as virtual substrates. ${ }^{[272]}$ With regard to approach (i), the sublattice orientation of these thin single-domain layers implies $\mathrm{Si}-\mathrm{P}$ bonds. This is confirmed by XPS: A second component in the $\mathrm{Si}$ and $\mathrm{P}$ photoemission lines can be ascribed to roughly $1 \mathrm{ML}$ of $\mathrm{Si}-\mathrm{P}$ bonds at the heterointerface. ${ }^{[272,275]} \mathrm{Si}-\mathrm{Ga}$ bonds cannot entirely be excluded, but their contribution is only very weak if any. ${ }^{[272,275]}$

Both $\mathrm{Si}$ and $\mathrm{GaP}$ surface preparation, as well as GaP nucleation can be controlled in situ with RAS. This seems of outmost importance to compare results obtained under different conditions in different reactors. Interface roughening was observed ex situ in recent work on $\mathrm{GaP}$ nucleation on almost nominal $\mathrm{Si}(100)$ and explained by a general faceting mechanism of the interface due to energetics. ${ }^{[282]}$ This generalization and the performed DFT calculations, however, were questioned, ${ }^{[283]}$ and the transfer from the used planar slabs ${ }^{[282]}$ to real facets may not be trivial.

\subsubsection{RAS of virtual GaP/Si(100) substrates and APD quantification}


Pseudomorphic $\mathrm{GaP} / \mathrm{Si}(100)$ heterostructures exhibit RA spectra similar to $\mathrm{GaP}(100)$ since the main spectral features can be assigned to identical surface reconstructions, i.e., either Prich $(2 \times 2) / c(4 \times 2)$ or the Ga-rich $(2 \times 4) .{ }^{[68]}$ By decomposing the spectral contributions, it was shown that the pure surface dielectric anisotropy of P-rich GaP/Si(100) indeed matches that one of P-rich $\mathrm{GaP}(100) .{ }^{[243]}$ Modifications of the lineshape due to interference and the anisotropy of the heterointerface (see above), however, are significant below the $\mathrm{E}_{1}$ interband transition of GaP. ${ }^{[68,243]}$ The intensity is additionally affected if antiphase disorder is present at the $\mathrm{GaP} / \mathrm{Si}(100)$ surface: Antiphase disorder in the GaP epilayer implies surface domains of mutually perpendicular dimer domains. ${ }^{[218]}$ Similar to the quantification of the domain content at $\mathrm{Si}(100)$ surfaces, ${ }^{[191,193]}$ this causes a decreased intensity of the RAS signal. ${ }^{[18]}$ Since $\mathrm{GaP}(100)$ exclusively shows bilayer steps, it can act as single-domain reference for scaling the spectra and thus the antiphase domain (APD) content can be obtained in situ. For correct scaling, however, interference due to internal reflection must be considered, which can be done either with an empirical approach ${ }^{[68]}$ or by fitting. ${ }^{[243]}$ Besides several ex situ approaches, ${ }^{[156,284,285,286,287,288]}$ APDs have also been observed with in system low energy electron microscopy ${ }^{[289,290]}$ and second harmonic generation (SHG). ${ }^{[291,292,293]}$ APDs at the buried interface also contribute to the interfacial electric fields. ${ }^{[294]}$

It is important to note that the APD content at the GaP/Si(100) surface is not necessarily identical to the domain imbalance at the $\mathrm{Si}(100)$ surface, since antiphase boundaries may annihilate during GaP growth by kinking. ${ }^{[262,295,296,297]}$ The kinking depends on growth conditions, ${ }^{[296]}$ and straight propagation is required to study the $\mathrm{Si}(100)$ surface indirectly. ${ }^{[298]}$ For entirely APD-free GaP epilayers, single-domain $\mathrm{Si}(100)$ surfaces are required. ${ }^{[155]}$ Singledomain RA spectra of GaP surfaces grown on preferentially A-type and B-type $\mathrm{Si}(100)$ surfaces, respectively, exhibit the opposite sign, which is caused by a sublattice inversion. ${ }^{[198]}$

Fig. 21 juxtaposes RA spectra of differently terminated $\mathrm{Si}(100)$ surfaces directly prior GaP nucleation and RA spectra of P-rich $\mathrm{GaP} / \mathrm{Si}(100)$ obtained after $\mathrm{GaP}$ growth on the corresponding $\operatorname{Si}(100)$ surface. It can clearly be seen that the sublattice orientation of the majority domain in the GaP layer is determined by the prevalent dimer orientation on the $\mathrm{Si}(100)$. The corresponding interface models are sketched in Fig. 21 assuming abrupt interfaces.

\subsubsection{GaP nucleation in presence of As}

$\mathrm{GaP}$ nucleation on As-modified $\mathrm{Si}(100)$ is studied less intense compared to monohydrideterminated $\mathrm{Si}(100)$. Recently, however, MOVPE growth of single-domain GaP epilayers on $\mathrm{Si}(100)$ :As was reported. ${ }^{[208,209]}$ Compared to GaP nucleation on monohydride-terminated $\mathrm{Si}(100)$, the GaP sublattice was inverted, as verified with $\mathrm{LEED}^{[209]}$ and RAS. ${ }^{[208]}$ Besides Si dimer orientation and ( $\mathrm{P}, \mathrm{Ga})$ chemical potential during nucleation (see above), As modification thus is another possibility to choose the desired sublattice orientation. Moreover, due to the "pre-nucleation" of the Si surface with As, GaP nucleation on Si:As may not require a dedicated low-temperature nucleation step. At least this step was omitted in Ref. [209] and recent TEM studies demonstrate that As-modification of the $\operatorname{Si}(100)$ surface prior high temperature GaP nucleation reduces the defect density induced at the interface drastically. ${ }^{\text {[299] }}$ When HF-dipping is applied rather than a sufficient thermal treatment to remove oxides from the surface, ${ }^{[199]}$ annealing in As helps to reduce defects, which are assumed to originate from remaining contamination on the $\mathrm{Si}$ surface after HF dipping. ${ }^{[201]}$ Possibly, prior Astermination will also have impact on the degradation of the bulk Si lifetime, which is often decreased in III/V MOVPE reactors. ${ }^{[300]}$ XPS studies, however, imply increased intermixing, ${ }^{[208]}$ so that the atomic structure of the GaP/Si:As interface may be more complex than in the $\mathrm{Si}-\mathrm{P}$ case discussed above. Also preliminary results on the GaP/Si(100):As interfacial 
dielectric anisotropy indicate an additional contribution. ${ }^{[301]}$ Such less abrupt interfaces may enable the formation of an equal number of $\mathrm{Si}-\mathrm{As}$ and $\mathrm{Si}-\mathrm{Ga}$, which would be beneficial with regard to interfacial charge compensation. ${ }^{[27]}$

\section{Surfaces modified by adsorbates from gas-phase}

Post-growth handling of devices and their application typically means that at least one surface is exposed to gases. While nitrogen in ambient air is generally acting as an inert gas, oxygen and water vapor can be considered the two most prominent reactive species that lead to the formation of new surface species. Ideally, these would passivate surface states acting as charge-carrier recombination states. But in reality, the modified surface often develops new charge-carrier traps. Studies on adsorption and reaction mechanisms from the gas phase reveal the formation of these species and corrosion mechanisms as a function of well-controlled surface properties. As the in situ access to the semiconductor-liquid interface at sufficient surface sensitivity in an electrolyte is experimentally rather challenging, gas-phase adsorption experiments in UHV also constitute a possibility to bridge the gap between liquid environments and very well-defined surfaces in UHV. Depending on surface and adsorbate, they can reveal surface reactions that still persist at elevated pressures. Reliable analysis and reproducibility for these experiments greatly benefit if the starting point for adsorption experiments is a well-characterized surface such as described in sections 2 and 3 above.

The same in situ techniques as mentioned in section 2 can be employed here as well as vacuum-derived spectroscopy, such as near-ambient pressure XPS (NAP-XPS). ${ }^{[302]}$ Time resolution combined with very high surface sensitivity is key to also allow for the analysis of reaction dynamics. In the following, we will review adsorption studies on some of the semiconductor surface systems treated above with a focus on water and oxygen exposure. We will see that reaction paths vary greatly with respect to the initial surface configuration and connect more traditional vacuum-based adsorption with recent trends in (near-)ambient pressure experiments.

\subsection{General aspects}

When molecules adsorb on a solid surface from the gas phase, one can distinguish three different ways in which the molecules attach to the surface:

(a) In the case of physisorption, the molecules do not form a covalent or ionic bond with the surface, bonding is of van-der-Waals type such as in the case of water on $\mathrm{TiSe}_{2 .}{ }^{[303]}$

(b) Dissociative chemisorption denotes the case where the molecule breaks apart and at least one fragment forms a covalent bond with the surface, a prototype reaction is the oxidation of a surface from molecular oxygen. ${ }^{[304]}$

(c) During non-dissociative chemisorption, a covalent bond is formed between adsorbate and surface, but the adsorbed molecule stays intact. An example here is water forming a covalent bond to a $\mathrm{Si}(100)$ surface by means of an oxygen lone pair. ${ }^{[305]}$

Coadsorption, where another species is adsorbed in a more or less rigid way, can greatly impact the effective surface interaction via blocking of sites or the promotion of dissociation, ${ }^{[306,307]}$ which is the motive that clean and well-defined surfaces are of essential importance here. Two significant, inter-related quantities for the description of adsorption behavior are the sticking coefficient and the effective coverage in monolayers. The magnitude of the sticking coefficient gives the ratio between adsorbate molecules that stick on the surface to the impinging ones. The coefficient can be determined by the analysis of the 
saturation behavior of surface properties directly related to the adsorbate quantity of the surface as Langmuir isotherms or by molecular beam adsorption. ${ }^{[306,308,309]}$ In the model of Langmuir adsorption, the surface coverage increases monotonically with time, where the perfectly planar surface with a specific reactivity of each surface site reacts with the adsorbate under isothermal conditions. ${ }^{[306,308]}$ Sticking coefficients cover several orders of magnitude, in the case of water from $10^{-4}$ for transition metal dichalcogenides to unity for $\mathrm{Si}(100)$ $(2 \times 1) .{ }^{[305]}$ Non-Langmuir behavior arises when the adsorbate on adjacent surface sites is interacting. ${ }^{[306]}$

The coverage $q$ is typically defined in monolayers. The term monolayer (ML) can, however, be defined in various ways. From the perspective of the substrate, one ML is the occupation of every bulk basis site lying in the surface plane. In the case of cubic (100) surfaces discussed in sections 2 and 3, the thickness of the ML is then a quarter of the lattice constant. From an adsorbate perspective, one can define one ML as a layer with the bulk density and thickness of one molecule. The quantity $q$ can then be estimated by means of quantitative XPS: ${ }^{[50]}$

$$
q=\frac{I}{e^{-d / \lambda_{0}\left(E_{O}\right) \cos (\theta)}+I \cdot e^{-d / \lambda_{0}\left(E_{S}\right) \cos (\theta)}-I-1} \quad .
$$

Here, $d$ is the overlayer thickness, $\lambda_{0}$ the electron attenuation length of the overlayer, ${ }^{[310]}$ $E_{S}, E_{o}$ the kinetic photoelectron energies of substrate and overlayer and $\theta$ the angle against normal emission. The term $I=I_{o, r e l} / I_{s, \text { rel }}$ is defined via the ratio $I_{o, s, r e l}=I_{o, s} / I_{o, s}^{\infty}$ of measured intensity $I_{o, s}$ against the calculated intensity of an infinitely thick layer $I_{o, s}^{\infty}$.

Temperature is an important parameter in adsorption experiments. In the case of cleaved $\mathrm{InP}(110)$ surfaces, water adsorbs dissociatively at a temperature of $100 \mathrm{~K}$ with a near-unity sticking coefficient before at higher exposures an ice layer is observed. ${ }^{[311]}$ Molecular water desorbs already at $150 \mathrm{~K}$, and at room temperature "annealing", In oxide forms, with the oxygen substituting for the $\mathrm{P}$. This demonstrates a wealth of temperature-dependent reactions of adsorbates with surfaces, but with the solid-liquid interface in mind, we will focus in the following on room temperature processes.

\subsection{Silicon surfaces exposed to water, oxygen, and hydrogen}

Silicon is probably the most intensively studied semiconductor surface regarding adsorption processes. This is partly owed to the fact that bulk crystals are readily available in highest quality and well-defined surface terminations can be prepared by various methods, ranging from annealing in UHV to wet-chemical etching. ${ }^{[305,312]}$ From an application point of view, the oxidation of $\mathrm{Si}$ is part of MOS technology and high-quality surface oxides benefit the device performance, moving critical interfaces to the interior of the device. ${ }^{[313,314,315]}$

Early experiments investigating water adsorption on $\mathrm{Si}$ relied on separate water adsorption followed by probing the modified surface with photoelectron spectroscopy. It was found that for instance the $(2 \times 1)$ reconstructed $\mathrm{Si}(100)$ surface features non-dissociative chemisorption of $\mathrm{H}_{2} \mathrm{O}$ with the oxygen oriented towards the surface at room temperature, but only in the case of well-ordered surfaces. ${ }^{[305]}$ Less ordered surfaces show a dissociative chemisorption behavior forming $\mathrm{OH}$ bonds. ${ }^{[316]}$ Unlike chemisorption of $\mathrm{O}_{2}$, where the coverage is higher and the oxygen penetrates below the surface, the surface coverage is limited here to about half a monolayer ${ }^{[305]}$ Such a behavior cannot, however, be generalized to other Si surfaces as each 
one exhibits a distinct reaction kinetics and activity of surface sites, ${ }^{[317]}$ emphasizing the requirement for well-ordered surfaces to study reaction mechanisms.

Reaction kinetics during adsorption can be revealed by means of time-resolved in situ spectroscopy, for instance RAS, but also other optical and scanning probe techniques. ${ }^{[306]}$ Due to the quantitative nature of RAS, the signal is directly proportional to the number of unaltered surface sites and one can derive the thermodynamics of Langmuir-like processes. As shown in Fig. 22, the main spectral features of the clean $(2 \times 1) \operatorname{Si}(100)$ surface are centered around the critical points of the Si band structure. With modifications by the adsorbate, signal shapes vary greatly for the different adsorbates such as molecular oxygen, benzene or water for the $\mathrm{Si}(100)$ surface. ${ }^{[318]}$ This shows that the optical signals are a convolution of optical anisotropy from the surface itself and the adsorbate molecule, which poses the challenge to distinguish and to identify the separate contributions from the experimental spectra. By means of in system STM, Witkowski et al. ${ }^{[318]}$ found that water on non-hydrogenated (2x1) Si(100) adsorbs dissociatively, with $\mathrm{OH}$ attaching to one half of the dimer, and the remaining $\mathrm{H}$ on the other Si atom. This does to some extent contradict the findings of Schmeisser et al ${ }^{[305]}$ where non-dissociative adsorption was found. The reason for the discrepancy could be a) that the adsorption process itself by Schmeisser et al. was carried out at low temperature or b) a different step density (similar to section 2.6.1), as Witkowski et al. used Si wafers with a higher miscut and, thus, increasing steps on the surface, while Schmeisser et al. did not report on this property of their samples.

The strength of bonding between surface and adsorbate can be evaluated by desorption experiments, either in the form of temperature-programmed desorption or by using the energy of an intense (laser) light pulse to remove the surface species. In temperature-programmed desorption experiments, the evaporated adsorbates or their fragments can be probed by in situ by mass spectrometry, which allows for an identification of desorbed species, but does neither give spatial information nor direct information on the surface itself. In single-shot laser induced thermal desorption, on the other hand, the surface is analyzed by STM after desorption, which is feasible as the desorption process is frozen and the surface species not smeared out by diffusion, enabling high spatial resolution. ${ }^{[319,320]}$

\subsection{InP surfaces}

InP has been used in solar water splitting applications for a long time, where its surface is inherently in close contact with water. ${ }^{[321]}$ More recently, the $(2 \times 4) \operatorname{InP}(100)$ surface (see section 2.1) has been the starting point for a very efficient and stable photocathode. ${ }^{[16,322]}$ The question arises whether the surface reconstruction of InP impacts the initial surface oxidation and if this is relevant for application of direct solar water splitting (solar-driven water photolysis).

For the oxidation of InP(100) surfaces by molecular oxygen, Chen et al. prepared the In-rich and the P-rich surface of $\operatorname{InP}(100)$, exposing them at different temperatures to oxygen pressures in the order of $10^{-5}$ mbar. $^{[304]}$ In situ RAS combined with in system photoelectron spectroscopy allowed them to correlate optical signatures to the oxygen uptake of the surface. They found that at room temperature the P-rich surface exhibits a much slower oxygen uptake rate than the In-rich surface. After a dose of $10 \mathrm{~kL}$, the oxygen coverage reached ca. $1 \mathrm{ML}$ for the In-rich surface, but only 0.1 ML for the P-rich surface. The oxygen uptake rate of P-rich $\mathrm{InP}(100)$ does, however, drastically increase for temperatures above $500 \mathrm{~K}$. Yet even then, some optical anisotropy of the surface persists, which indicates that the surface does not complete lose its ordering. The In-rich surface, however, becomes almost completely 
optically isotropic already at $300 \mathrm{~K}$. The results were interpreted as a dissociative chemisorption of $\mathrm{O}_{2}$, inserting the oxygen in between both $\mathrm{In}-\mathrm{In}$ and $\mathrm{In}-\mathrm{P}$ bonds. ${ }^{[04]}$

To study the adsorption of water and to evaluate potential differences to oxygen, we prepared $\operatorname{InP}(100)$ surfaces as described in section 2.1 and transferred them contamination-free to a UHV cluster equipped with XPS, LEED, and a separate adsorption chamber with an optical port for in situ RAS. ${ }^{[50]}$ Fig. 23(a) shows the resulting time-resolved spectrum for water exposure at room temperature. We see that, unlike for oxygen exposure (Fig. 23(b)), the optical anisotropy of the surface is mostly conserved. The high-energetic feature $I 5$ related to a surface-modified bulk transition is even completely conserved, which shows that the water does not lead to subsurface oxidation, even after a water dose of $25 \mathrm{~kL}$. Analysis of the resulting surfaces by XPS (Fig. 24) shows that the predominant $\mathrm{O} 1 \mathrm{~s}$ feature for $\mathrm{H}_{2} \mathrm{O}$ exposure is an oxygen atom in between In and $\mathrm{P}$ (labelled $\mathrm{O}_{\mathrm{I}}$ ) with almost no $\mathrm{In}-\mathrm{O}$ - In bonds, while for $\mathrm{O}_{2}$ exposure, the $\mathrm{In}-\mathrm{O}$ - In motive (labelled $\mathrm{O}_{\mathrm{II}}$ ) becomes much stronger. These assignments were corroborated by ultraviolet photoelectron spectroscopy of the P $3 p$ and $\mathrm{In} 4 \mathrm{~d}$ lines, where both adsorbates induced a modification of the $\mathrm{P} 3 \mathrm{p}$ line and the $\mathrm{O} 2$ exposure a relatively stronger oxide peak in the In $4 \mathrm{~d}$ line. ${ }^{[50]}$ No evidence for $\mathrm{OH}$ groups was found, so we assume a complete dissociation of the water. There is, however, a small signal at $527.8 \mathrm{eV}$ binding energy, which might originate from oxygen bound to surface defects. Quantitative analysis after eq. (1) shows ca. $0.25 \mathrm{ML}$ oxygen after an $\mathrm{H}_{2} \mathrm{O}$ dose of $25 \mathrm{~kL}$, and ca. 0.6 ML after $15 \mathrm{~kL}$ of $\mathrm{O}_{2}$. The latter quantification is, however, subject to a relatively large error due to the subsurface diffusion of oxygen. The stability of the oxide was evaluated using temperature-programmed desorption. A transfer of the samples back to the MOVPE reactor followed by annealing under RAS control showed that the In-rich surface modified by water could be completely restored by gentle annealing to $570 \mathrm{~K}$ without the supply of precursors, while in the case of $\mathrm{O}_{2}$ exposure, the initial optical anisotropy could not be recovered. These findings were also corroborated by UPS, where the valence band spectrum was also found to be completely recovered after annealing of the water-exposed surface. UPS also confirmed the evolution of a pronounced surface dipole for oxygen exposure $(0.3 \mathrm{eV}$ shift of the work function) and a weaker dipole for the water-modified surface $(70 \mathrm{meV}){ }^{[50]}$ Features as the orientation of the water molecule on the surface can, depending on the system, also directly derived from RAS if suitable calculations are available. ${ }^{[323]}$

A DFT study on water adsorbed to the In-rich surface by Wood et al.$^{[324]}$ showed that In$\mathrm{O}$ - In bonds create in-gap states leading to surface charge carrier recombination, while In$\mathrm{O}-\mathrm{P}$ bonds avoid the trap state. Consequently, it appears that water exposure electronically passivates the In-rich $\operatorname{InP}(100)$ surface, while oxygen exposure does not. The passivation is reversible, as the oxygen is only weakly bound to the surface, similar to "epi-ready" InP growth substrates. This finding explains the success of electrochemical in situ functionalization procedures for InP photocathodes, ${ }^{[16]}$ where the surface is modified in an aqueous electrolyte and motivated the development of an electrochemical in situ surface modification routine for an AlInP surface of a photoelectrochemical tandem cell. ${ }^{[14]}$ Later, an electronic surface passivation of In-based surfaces by water was also confirmed by in situ photoluminescence measurements on GaInP that enabled the observation of the electronic performance during water exposure. ${ }^{[325]}$

The P-rich $\operatorname{InP}(100)$ surface is, unlike for oxygen exposure, ${ }^{[304]}$ surprisingly unstable upon water adsorption. ${ }^{[50]}$ Thereby, the optical anisotropy of P-rich surfaces is irreversibly destroyed. Specific sites of the surface reconstruction being attacked by the polar water molecule could be the polar $\mathrm{P}-\mathrm{H}$ bond or the lone pair of the $\mathrm{P}$-dimer. This again 
emphasizes that the initial surface reconstruction has a great impact on the initial interaction of $\operatorname{InP}(100)$ surfaces with adsorbates.

\subsection{GaP surfaces}

$\mathrm{GaP}(100)$ surfaces are structurally and electronically closely related to $\mathrm{InP}(100)$ surfaces. Yet their contact with water is not passivating the surface as in the case of InP, but leads to an unfavorable oxide, which hampers application in solar water splitting due to an unfavorable internal band offset between oxide and bulk. ${ }^{[59]}$ Again, we prepared well-defined, Ga-rich and P-rich $\mathrm{GaP}(100)$ surfaces to study their adsorption behavior with respect to water and oxygen. Fig. 25 juxtaposes in situ RAS of P-rich and Ga-rich $\mathrm{GaP}(100)$ during exposure to water. ${ }^{[326]}$ We see that in both cases, some anisotropy is conserved and for the Ga-rich surface, the surface modified bulk transition as well, similar to the observations for In-rich $\operatorname{InP}(100)$. The Ga-rich surface is more reactive, as indicated by a more rapid reduction of the low-energy peak in the spectrum. A closer look on the RAS signal of the P-rich surface after exposure of water reveals the evolution of a new negative peak $P 3$ around $4.5 \mathrm{eV}$ (Fig. 26(a)). This peak is associated with a new $\mathrm{c}(2 \times 2)$ surface reconstruction as revealed by LEED (blue-framed inset of Fig. 26(a)).

Surprisingly, the new surface geometry does not involve any oxygen as it was evidenced by XPS (Fig. 26(b)). Hence water itself or the dissociated fragments oxygen or hydroxyl groups are not involved in the reordering of the surface. The lack of oxygen in the surface still holds true for the highest applied exposures of more than $100 \mathrm{~kL}$ and reveals an extraordinary inertness of the P-rich surface against oxidation by water, in stark contrast to the P-rich InP surface. The new superstructure could arise from a full dissociation of the water molecule, releasing oxygen and hydrogenating the surface, similar to what was found for hydrogenexposed P-rich InP. ${ }^{[327]}$ Further experimental and theoretical analysis is, however, required to understand this surface behavior and its spectral features.

The Ga-rich surface, on the other hand, features a mixture of dissociative chemisorption of water, forming hydroxyl groups, combined with the co-adsorption of molecular water. ${ }^{[326]}$ This trend was confirmed by near-ambient pressure X-ray photoelectron spectroscopy of 'sputter-annealed' GaP surfaces. ${ }^{[328,329]}$ In NAP-XPS, a differentially pumped photoelectron spectrometer with a small aperture is brought very close to the sample surface, which allows for exposing the sample to pressures in the order of 10 mbar during the measurement. ${ }^{[302]} \mathrm{By}$ these means, the chemical composition can be monitored in situ as a function of the pressure, further approaching ambient conditions at room temperature.

Kronawitter et al. ${ }^{[328]}$ studied sputtered GaP(111) surfaces and found for pressures up to $10^{-4}$ mbar the same mixture of hydroxyl groups and co-adsorbed molecular water as for the Garich (100) surface, ${ }^{[326]}$ which evidences some degree of similarity of the two surfaces with respect to water adsorption. Beyond 0.3 mbar, they find an additional photoelectron peak which they ascribe to interaction between $\mathrm{OH}$ and $\mathrm{H}_{2} \mathrm{O}$ species. Zhang and Ptasinska investigated sputtered $\mathrm{GaP}(110)$ surfaces by NAP-XPS, also finding hydroxyl groups and molecular water, but, in addition, two more contributions, including $\mathrm{Ga}-\mathrm{O}-\mathrm{Ga}^{\left[{ }^{[329]}\right.}$ This could indicate that the $\mathrm{Ga}(111)$ surface is more prone to direct oxidation by water. Both studies do, unfortunately, lack in situ and also in system control of the surface after or rather during the sputtering procedure, e.g., by LEED, which can in principle lead to an overestimation of the reactivity of the surface due to defects ${ }^{[305]}$ and contaminants such as carbon.

\subsection{In situ studies on noble metal surfaces}


Noble metal surfaces are of interest for energy conversion applications, as they are very often used as (co-)catalysts in solar fuel production. A wealth of adsorption studies has been conducted regarding water, but also organic compounds, probed in situ by methods such as Xray absorption near edge structure (XANES), LEED or surface enhanced Raman spectroscopy. ${ }^{[306,330,331,332]}$ The information from these experiments provided crucial input for the development of theoretical methods to describe heterogeneous catalysis. ${ }^{[333]} \mathrm{A}$ famous example are oscillatory processes during CO oxidation on Pt surfaces. ${ }^{[330]}$ While it is beyond the scope of present review to cover the literature with respect to adsorption studies and we refer the reader to reviews in the literature, ${ }^{[306,334]}$ we would like to highlight the extension of RAS in the form of Reflection Anisotropy Microscopy (RAM) as another example of in situ analysis to investigate $\mathrm{CO}$ oxidation on $\mathrm{Pt}(110) .{ }^{[335]}$ Punckt et al. combined RAS with optical imaging, achieving spatial resolutions of better than $10 \mu \mathrm{m}$ and a temporal resolution of 40 ms. ${ }^{[35]}$ This setup revealed structural aspects of CO-poisoned islands of the $\mathrm{Pt}(110)$ surface and catalytic properties resolved in time and space. This technique is consequently also interesting for studies of catalytic properties at electrochemical interfaces.

\section{The solid-liquid interface}

Further complexity in interface formation arises when liquids are introduced. The solid-liquid interface combines the world of the solid state with its crystal structure and extended Bloch states with the interfacial reactions and the dynamics of liquid environments and their redox levels. For heterogeneous catalysis, this interface is the key aspect. ${ }^{[333]}$ The Helmholtz-layer with its specifically adsorbed ions, the diffuse double layer and the resulting potential drop in solid and electrolyte are closely connected to the surface properties of the solid. ${ }^{[336,337]}$ Corrosion sets in here and in catalysis or solar water splitting, charges have to be transferred over the solid-liquid interface. ${ }^{[338]}$ Especially in the case of semiconductors, where additional properties such as space-charge layers and quasi-Fermi levels under illumination arise, the solid-liquid interface still holds many open questions. In the following, we will briefly review some in situ spectroscopy methods with spatial resolution applied to semiconductorelectrolyte interfaces relevant for solar energy conversion and give an overview of recent methodological developments. Besides the epitaxial materials discussed in previous sections, metals are often applied as electrodes for water splitting and will partly be covered here as well.

The most common technique in electrochemistry is voltammetry, where the current passing through an electrode into the electrolyte is recorded as a function of the potential with respect to a reference such as a standard hydrogen electrode. ${ }^{[339]}$ The technique is intrinsically in situ and provides feedback on energetics, kinetics, and in case of well-defined systems submonolayer resolution for structurally induced features. A famous example are the surface reconstructions of $\mathrm{Au}$ single crystals, giving rise to specific signals. ${ }^{[340]}$ To reveal chargecarrier dynamics in illuminated semiconductors, intensity modulated photocurrent spectroscopy can be employed. Here, the photocurrent of a semiconductor is treated as a timedependent perturbation. A similar technique is (photo)electrochemical impedance spectroscopy, where the applied potential is perturbed under constant illumination. ${ }^{[341]}$ These in situ techniques based on potential and current measurements do not directly provide spatial resolution or chemical information to analyze corrosion processes or bond formation in catalysis. So they have to be combined either with in system or ex situ analysis. An example of the latter one is shown in Fig. 27, where illumination of the surface of an AlInP layer, which acts as window layer of a tandem absorber, initiated an oscillation of the open-circuit potential (OCP). Due to the lack of spatial resolution, the measured OCP is an average over the whole sample surface and from a well-defined oscillation, it follows that the whole surface is in the same state, which enables ex situ analysis. At the local extrema of the oscillation, the 
sample was brought to ex situ XPS to probe the state of the surface. Fig. 27(b) shows that at the maximum of the oscillation, the oxide layer reaches a minimum, while at the minimum potential, the oxide layer is ca. $0.4 \mathrm{~nm}$ thicker. ${ }^{[14]}$ The oscillatory process is a photoelectrochemical layer-by-layer etching process, as the AlInP layer disappears after a number of oscillations, similar to the Si etching in MOVPE ambient described above (Fig. 15). At the minimum surface oxide content, the process was aborted and directly followed by photoelectrochemical catalyst nanoparticle deposition, avoiding exposure to oxygen from ambient air, which would create charge-carrier recombination centers as discussed above. This step turned out to be crucial for the device performance, leading to an electronically and chemically passivated surface and hereby enabling efficient solar water splitting. It should be noted that such an oscillatory process, where the whole macroscopic, immersed surface is in the same microscopic phase, requires an atomically well-defined surface with a minimum of defects, for instance epitaxial layers as described in sections 2 and 3. An energy schematic for the resulting tandem device is presented in Fig. 28, showing the band alignment of the heterostructure under illumination. Charges have to be transferred from the GaInP absorber of the top cell across the window layer to the phosphate layer and from there to the electrolyte supported by the catalyst. While the first buried interface between GaInP and AlInP is welldefined from growth, ${ }^{[342]}$ the interface between highly n-doped GaInP and the $\mathrm{PO}_{\mathrm{x}}$ species has to be adequately aligned to allow an efficient charge transfer. The energetic alignment between $\mathrm{PO}_{\mathrm{x}}$ and the $\mathrm{Rh}$ nanoparticles is dominated by the n-type phosphate layer as long as the Rh nanoparticles are small and weakly interconnected. ${ }^{[14]}$ This does, however, also require the $\mathrm{PO}_{\mathrm{x}}$ compounds on the surface to be (electro)chemically stable, as the $\mathrm{Rh}$ nanoparticles cannot screen them completely from exposure to the electrolyte.

Oscillating electrochemical interfaces are, however, best studied by in situ methods to provide better temporal resolution and to ensure that the state of the surface is not perturbed by removal of the electrolyte during the transfer to subsequent analysis. Apart from the $\mathrm{CO}$ oscillations at Pt surfaces mentioned above, ${ }^{[330]}$ which might be relevant for $\mathrm{CO}_{2}$ reduction applications, the oxidation of $\mathrm{Si}$ in the electrolyte is also a prominent case of an oscillating electrochemical interface. Miethe and Krischer ${ }^{[343]}$ studied the anodic oxidation of Si in a fluoride-containing electrolyte by means of in situ ellipsometry. They find that the dissolution of p-type $\mathrm{Si}$ is spatially uniform, while n-type $\mathrm{Si}$ exhibits a patterned behavior.

Let us revisit the $\mathrm{Au}$ surface, which is a system well-suited for fundamental studies and method development, as it can be transferred to the electrolyte in a well-defined surface condition and is also stable in the liquid. Surface-enhanced infrared absorption spectroscopy employed by Ataka et al. ${ }^{[344]}$ revealed the orientation of water molecules at the $\mathrm{Au}(111)$ surface as a function of the potential. From potentials below to above the potential of zero charge, water molecules reorient from the two hydrogen atoms close to the surface to hydrogen towards the solution, forming an ice-like structure with a following second layer of water.

Smith et al. studied the $\mathrm{Au}(110)$ surface by means of electrochemical RAS. ${ }^{[345,346]}$ They identified signal shapes as a function of electrolyte and applied potential and found that the (1x3) $\mathrm{Au}(110)$ surface decays over time due to the accumulation of impurities at the interface (Fig. 29). Time evolution of the optical anisotropy showed that the spectral features reveal a distinct decay behavior, related to the specific kinetics. They indicate that the impurities accumulating at the surface can be removed from the surface by a short positive potential pulse restoring the original spectrum. Their experiments demonstrate that RAS can also be a probe of surface species in the liquid electrolyte, but also show that the interpretation of spectra is very difficult, if there is no backing from theoretically derived spectroscopy. 
Spatial resolution —often at the cost of temporal resolution - can also be achieved by various scanning probe techniques such as scanning photocurrent microscopy or electrochemical scanning tunneling microscopy. ${ }^{[9]}$ These techniques do, however, often have the disadvantage of a very limited temporal resolution, which impairs in situ control during surface processing.

The combination of intrinsic electrochemical methods with in situ spectroscopy at sufficiently high time, energy and spatial resolution for process control can still be considered an evolving field. The future development of techniques and their adequate combination will help to further understand and precisely shape electrochemical interfaces.

\section{Photochemical reactions to control semiconductor / polymer / biological interfaces}

The solid-liquid interface was discussed above with focus on its application in artificial photosynthesis. Inspiration by nature is not only useful for the conversion of sunlight into storable types of energy: Nature may act as role model to construct even more complex, hybrid interfaces, where photoinduced interfacial reactions play an important role, a.o., in sensing applications. Novel strategies for intelligent materials and photo-induced interfacial reactions aim to build intelligent, i.e., bioactive, dynamic, non-equilibrium, materials, acting as biosensors or chips varying their time characteristics: so-called life-inspired nanoscale machineries. ${ }^{[347,348,349,350,351,352,353]}$ Strategies involve needs for effective energy conversion with the focus on oscillation reactions, ${ }^{[354]}$ chemical networks, ${ }^{[355]}$ autocatalytic ${ }^{[356]}$ and autoamplification $^{[357]}$ reactions, which mimic living systems ${ }^{[358]}$ using cell metabolic biomolecules $^{[359]}$ and ions, e.g.. protons. ${ }^{[360]}$ Biological systems solve such an energymanagement by developing unique sensory and adaptive capabilities, ${ }^{[361]}$ transport mechanisms guided with ions, ${ }^{[362]}$ proton gradients ${ }^{[363]}$ and chemical networks. ${ }^{[364]}$ It is very attractive to utilize light for the modulation ${ }^{[351,352]}$ of simple, reliable chemical reaction networks, because it is easy to control based on existing knowledge on reliable photosensitive material. The focus in this section is on well developed, reliable inorganic semiconductors and photo-induced surface reactions for new functions.

Intelligent interface architectures ${ }^{[365]}$ provide new concepts of biocide materials, coatings, biosensors and microfluidic chips. Our vision is to facilitate an in situ localization of gradients at certain sectors on a substrate to amplify or inhibit reactions and to control the polymer response for regulation of biomolecules, biofilms, and biosensing. In the following, we focus on the possibility of efficient transformation of energy of electromagnetic irradiation into local ion gradients to actuate soft matter and biofilm formation. The strategy we suggest here is to combine in situ control of both the generation of ion concentration gradients on the semiconductor surface and of the film formation with time and spatial resolution (Fig. 30) in order to achieve a network, which exhibits preferably several mechanisms for spatiotemporal switching, ${ }^{[366]}$ amplification or inhibition, ${ }^{[356]}$ activated depending on type and intensity of the external demand. ${ }^{[367]}[365]$

Apart from photo-induced formation of ion gradients, we suggest an attractive strategy of amplification of concentrations of ions. One proton can, for example, provide several protons by autocatalysis. Control of ion and proton concentration gradients may be achieved by assembly of soft matter on the semiconductor surface, e.g., polyelectrolytes (PEs) by layerby-layer (LbL) assembly. Prime issues are: (i) How many photons are needed to locally start the required chemical reaction on the surface? (ii) What is the optimum PEs LbL architecture to understand the basics of ion trapping and storage, the gradients under local irradiation? (iii) How to achieve reversible actuation, faster or slower, of different LbL assemblies with 
controlled response times for bioapplications? To answer these questions, it is important to investigate both the change in the material and the system response in situ.

It is a challenging task to control spatiotemporal system gradients. Ulasevich et al. ${ }^{[368]}$ proved that it is promising to use inorganic semiconductors to initiate a spatial-temporal gradient of

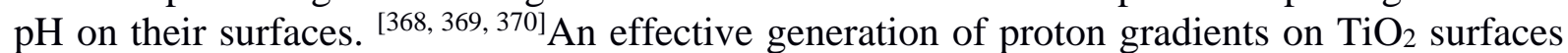
under irradiation has been introduced ${ }^{[368,369,370]}$ to control the bioactivity of the system for specific bioapplications of guiding cell migration on the surface ${ }^{[370]}$ for co-culturing biochips, and for protein recognition / modulation elements. ${ }^{[369]}$

For the specific examples and applications discussed here, the following key questions are important:

(a) How can the photogeneration of charges in a solid be used to initiate light-specific chemical reactions with time and space control? The question covers several topics, such as photon absorption, ${ }^{[371]}$ carrier generation and mobility, ${ }^{[372]}$ chemical conversion into reactive oxygen species (ROS),${ }^{[373]}$ ion gradients, ${ }^{[368]}$ autocatalytic species to adjust amplification of ion gradients, such as a proton gradient, ${ }^{[374]}$ as well as flexible positioning of the photoelectrode by the irradiation spot. ${ }^{[375]}$

(b) How do photogenerated ion gradients change the properties of the adjacent soft matter? The task is to "freeze" ion gradients locally, ${ }^{[376]}$ to actuate soft matter, ${ }^{\left[{ }^{[68]}\right.}$ to store ion carriers, ${ }^{[377]}$ to release ions into the PEs LbL matrix ${ }^{[369]}$ and to control lifetimes, oscillations of mechanical properties and LbL stability. Sub-questions then concern (i) the dependence on PEs LbL architecture; ${ }^{[378,379,380]}$ and (ii) the specificity for a semiconductor, effectiveness of doping as well as duration and intensity of irradiation. ${ }^{[381]}$

(c) How does specific ion release at localized areas and change in morphology of the surface affect bacteria attraction and biofilm growth? The question concerns synergetic network regulation and spatiotemporal surface bioactivity to attract, deactivate, and affect their metabolism and desorb bacteria. ${ }^{[382]}$ Sub-questions are then related to (i) the mechanisms behind the effect of ROS on the bacteria; ${ }^{[383]}$ (ii) the ion gradient to support growth of selective bacteria types located on certain areas; ${ }^{[384]}$ and (iii) the effects of the surface-modified films as the photo-controllable bioactuators such as conformational transitions in the polymeric film. ${ }^{[369,370]}$

Concerning possible ion concentration gradients running in a network in parallel on semiconductor surfaces, we can ask if there is a universal strategy to predict possible ion concentration gradients and to suggest nanoscale machineries with chemical networks to control biofilms based on generation of ROS on well-known and controllable inorganic semiconductors. Here, life-inspiration can be mentioned. ${ }^{[348,385]}$ For example, it is known that, in order to trigger inflammation, the $S$. Typhimurium population ${ }^{[364]}$ generates ROS with a subsequent production of electron $\left(\mathrm{e}^{-}\right)$acceptors. These electron acceptors enable $S$. Typhimurium to use nutrients in the anaerobic environment. Some life-inspired transfer chain reactions are mentioned in Fig. 30(a). It is interesting ${ }^{[364]}$ that ROS generated by the inflammatory are known to exhibit "sulfur" pathways as they oxidize thiosulfate to $\mathrm{S}_{4} \mathrm{O}_{6}{ }^{2-}$, providing a host-derived electron acceptor that supports growth of S. Typhimurium by anaerobic respiration. ${ }^{[386]}$ The reductase of S. Typhimurium may reduce the tetrathionate $\left(\mathrm{S}_{4} \mathrm{O}_{6}{ }^{2-}\right)$ to thiosulfate $\left(\mathrm{S}_{2} \mathrm{O}_{3}{ }^{2-}\right),{ }^{[387]}$ with further reduction to $\mathrm{H}_{2} \mathrm{~S}$ by sulfite reductase, ${ }^{[388]}$ and thiosulfate reductase. ${ }^{[389]}$ 
"Sulfur" pathways in cells mentioned above are not the only inorganic exogenous electron acceptor pathways generated during inflammation. ${ }^{[364]} \mathrm{NO}$ can react with superoxide $\left(\mathrm{O}_{2}{ }^{-} \cdot\right)$, a ROS to form $\mathrm{NO}_{3}{ }^{-}$that boosts growth of $S$. Typhimurium during gastroenteritis by nitrate respiration. ${ }^{[390]}$ The pathogen uses energy taxis to migrate to spatial niches containing hostderived nitrate or tetrathionate. ${ }^{[391]}$ How can we trace the pathogen on a semiconductor, e.g., $\mathrm{TiO}_{2}$ surface? We suggest using photosystems to trace various inorganic "food" for pathogens (Fig. 30(a)). We apply localized irradiation to trace their behavior or deactivate and detach them by localized ion concentration gradients, drug delivery and time-controlled actuation of soft matter. Such nanoscale artificial machineries with parallel chemical reaction networks are on the frontiers of future technologies to trace spatiotemporal biomolecules, microorganisms, and cell pathways.

Today, a few parallel chemical reaction networks have already been designed for photoreactions on semiconductors. Further, we will give vivid examples of spatiotemporal light triggered gradients of protons on semiconductor surfaces. ${ }^{[368]}$ Surprisingly there has been no data available to what extent the $\mathrm{pH}$ can be altered and how useful $\mathrm{pH}$ differences $(\Delta \mathrm{pH})$ can be before our recent study. ${ }^{[368]}$ It has been shown that the $\mathrm{pH}$ can decrease from 7 to 4 very quickly and locally in the irradiated spot. It is thus a promising idea to use light $-\mathrm{pH}$ coupling for modulation of $\mathrm{pH}$-sensitive organic molecules, and $\Delta \mathrm{pH}$ to modulate $\mathrm{pH}$ dependent soft matter with light on the surface of a semiconductor (Fig. 30(b)). ${ }^{[368]} \mathrm{pH}-$ dependent polymers are a class of materials with tremendous structural variety. ${ }^{[392]}$ One powerful example is "weak" PEs, ${ }^{[393]}$ including biopolymers ${ }^{[367]} \mathrm{pH}$ dependent polymers are also hydrogels, ${ }^{[394]}$ which exhibit pronounced morphological changes in response to $\Delta \mathrm{pH}$ and can also be used for multilayer formation. ${ }^{[395]}$ It is the hot topic of recent works ${ }^{[368]}$ to use LbL assemblies on photoactive $\mathrm{TiO}_{2}$ surfaces, presented schematically in Fig. 30(b) with the example of the successful modulation of LbL assembly thickness and with possible biomolecules, ${ }^{[369]}$ biofilm detachment, ${ }^{[382]}$ and cell migration ${ }^{[370]}$ due to film actuation and $\mathrm{pH}$ sensitive bonds in LbL assemblies. In the first measurements it was realized that the structural change induced by $\mathrm{pH}$ remains over hours after switching off the light and is advantageous for aims like cell growth and differentiation. ${ }^{[370]}$

One perspective is in situ modulation of photochemical processes on semiconductor surfaces. However, there are still a lot of challenges. Studies of the following issues are required to overcome existing scientific problems related to nanoscale electromagnetic energy transformation into ion concentration gradients: effects of nanoconfinement, chemical reaction networks in the system on formation of different ion gradients at localized areas under local irradiation of multipoints; energy exchange mechanisms in nanoscale energy storage and release systems; formation of isolated conditions for ion gradients to avoid uncontrolled interaction with ambient environment; effective separation of photo-generated ions over the surface; diffusion length of the photo-ions in PEs LbL multilayers in horizontal and vertical directions and others.

The other promising idea is that ion and proton concentration gradients can be amplified drastically (Fig. 30(b), inset) due to nonlinear organic reactions to proliferate acidic molecules in soft matter. ${ }^{[374]}$ We are sure, that this scientific field will grow further being unique to use reproducible light sensitive materials, e.g., inorganic semiconductors, for a new function of building blocks for nanoscale bio-machineries ${ }^{[353]}$ and biosensing. ${ }^{[369]}$ 


\section{In situ techniques for the detection of ROS and ion activity}

In this review, we focus on in situ observation of interfacial chemical reactions on nanostructured semiconductor surfaces, such as $\mathrm{TiO}_{2}$, involving reactive oxygen species (ROS) and ion generation, molecules generated in the system, such as hydroxyl radical, the singlet oxygen, "sulfur" pathways, polymer soft matter actuation and bioresponse by fluorescence assays, scanning ion-selective electrode technique (SIET), $\mu$-confocal photoluminescence ( $\mu$-CPL), and microorganism response. Advanced techniques are used to determine the spatiotemporal location of the photocatalytically active sites close to the surface. As an example, we summarize some examples of reactive compound detection in Table 1. It is interesting, that again life-inspiration ${ }^{[364]}$ can be used to find possible methods to detect ROS: ROS detection in a number of physiological ${ }^{[396,397,398,399]}$ processes significantly improved due to paying high attention to microbiology.

\subsection{Fluorescence assays}

In cell staining laser-induced fluorescence spectroscopy, fluorescent probes that correspond to exact processes are used. ${ }^{[400,401,402]}$ The ROS effects occur through reactions with a large variety of cellular components including proteins, nicotinamide adenine dinucleotidephosphate, cysteine, tryptophan, glutathione, tyrosine, ascorbic acid, and nucleic acids, cholesterol, fatty acids, which cause membrane lipid peroxidation. ${ }^{[403]}$ Nowadays many species to guide biological pathways in situ are commercially available. For example, some probes are known to generate or detect various ROS, including singlet oxygen $\left(1 \mathrm{O}_{2}\right)$, superoxide anion $\left(\cdot \mathrm{O}_{2}^{-}\right)$, hydroxyl radical $(\mathrm{HO} \bullet)$, various peroxides (ROOR') and hydroperoxides $(\mathrm{ROOH})$, as well as the nitric oxide radical $(\mathrm{NO}) \cdot{ }^{[404]}$

Several reviews discuss the chemistry of the different ROS and their fluorescence assays in photochemical processes. ${ }^{[404,405]} \mathrm{We}$ want to point out here, that in photochemistry the future focus cannot only be on detection of photogenerated species and reaction pathways, but also on their localization within a certain surface area and on the time resolution of photochemical processes. In particular, in Fig. 31, it is shown, that it is very important to study localization of gradients on the surface, e.g., ion gradients. ${ }^{[406]}$ A light-addressable photoelectrode suggested imaging with a fluorescence molecule and ion indicators. Fig. 31(b) describes an experimental setup for light-addressing imaging. $\mathrm{pH}$ imaging is possible with $\mathrm{pH}$ indicators, as shown in Fig. 31(c,d). In Ref. ${ }^{[370]}$, the fluorescent $\mathrm{pH}$ indicator 4,4'-(anthracene-9,10diyldimethanediyl)dimorpholine, which exhibits $\mathrm{pK}_{\mathrm{a}} \approx 5.1$, was used. Other indicators are easy to adopt to study ions and molecules on inorganic semiconductor surfaces, but again the tendency of today is to rely time and space resolution in order to enable studies of simultaneous reactions of different species, where each of one affects the other. Taking into account the recent progress in experimental studies of cell and cell metabolism, it is clear that parallel networks are more than realistic to adopt the assays for photochemical reactions.

\subsection{Scanning ion selective electrode technique (SIET)}

In situ SIET for mapping the photoactivity over the surface under local irradiation is new for the $\operatorname{area}^{[368,369,370]}$ and its further development should be beneficial to the large scientific community working in this field. In particular, in Ref.s ${ }^{[368,369,370]}$ the photogenerated proton activity over $\mathrm{TiO}_{2}$ surface was monitored, as displayed in Fig. 32. For the modulation of $\mathrm{pH}$ sensitive soft matter, biomolecules and microorganisms with light, it is important to understand how photo-initiated processes on the semiconductor $\mathrm{TiO}_{2}$ result in the transformation of light to an ion and in $\mathrm{pH}$ change, including localization of the effect. SIET is a very attractive technique with a sensitivity better than $\mathrm{pH} 0.2$ units for mapping of the activity and migration of $\mathrm{H}^{+}$ions on a $\mathrm{TiO}_{2}$ film in aqueous solution during local irradiation. SIET allows measurements of the concentration of specific ions (in particular in the 
mentioned case, $\mathrm{H}^{+}$ions) at a nearly constant microdistance over the surface. ${ }^{[407]}$ In Ref. ${ }^{[407]}$ it is also mentioned that photoactivity is an important issue. Thus, to detect clear signals it is advantageous to a mesoporous $\mathrm{TiO}_{2}$ layers with high photoactivity ${ }^{[368,408]}$ compared to the lower photoactivities discussed in the following for modulation of soft matter and biomolecules. ${ }^{[370]}$

SIET maps of a $\mathrm{TiO}_{2}$ surface before and after irradiation are shown in Fig. 32, confirming that it is possible for $\mathrm{TiO}_{2}$ to release protons upon irradiation. Fig. 32(b) displays, how the $\mathrm{pH}$ in the center of the irradiated spot varies when switching on off the local irradiation. The duration of irradiation correlates with the $\mathrm{pH}$ obtained: $5 \mathrm{~s}, 1 \mathrm{~min}$, and 3 min of irradiation result in $\mathrm{pH}$ of 5.6, 4.5, and 4.0 peak values, respectively. The action of different photoelectrochemical reactions (Fig. 30(b)) on the $\mathrm{TiO}_{2}$ surface under illumination seems to be the only plausible explanation for the observed local acidification of the solution and confirms that $\mathrm{TiO}_{2}$ is promising to demonstrate our concept to run reactions in parallel on a semiconductor to have ion and molecule gradients in time and space.

It is interesting to perform experiments with mobile flexible photoelectrode positions, where two or more semiconductor electrodes are placed in one Petri dish, and to shine the light on one of them or irradiate simultaneously several spots on the surface at different distance/location, with different intensity and duration. The flexible spatial addressability, oscillations, and reversible changes are great advantages for nanoscale machineries. The next step may be the assembly of the actuating polymer soft matter on low-photoactive semiconductor surfaces by changing parameters in the system (Fig. 30).

\section{$7.3 \mu$-Confocal Photoluminescence ( $\mu$-CPL)}

One more technique that allows in situ monitoring of local changes are photoluminescence (PL) measurements with 3D micrometer resolution on nanostructured semiconductors: $\mu$ CPL. ${ }^{[409]}$ Again, its development should benefit the large scientific community working in this field. In particular, in Ref. ${ }^{[409]}$ the formation of mesoporous silicon by high intensity ultrasonic surface treatment was established (Fig. 33) and displayed a strong PL activity. Numerous models ${ }^{[410,411,412]}$ can be proposed to explain the PL of the modified silicon, including quantum confinement, surface states, defects in the oxide, and the formation of hydrogen-terminated bonds. It is important to study the nature of PL centers, that can be changed in time, in situ ${ }^{[409]}$. One can monitor the PL signal in situ during ageing of nanostructured silicon (Fig. 33(a,b)), going from a non-oxidized structure to a partially oxidized one. Radiative recombination takes place within the surface amorphous layer (Fig. 33(c)). Surface states localize electrons and holes, either separately or together with the formation of nanocrystalline (nc)-Si in the porous matrix of $\mathrm{SiO}_{\mathrm{x}}$. One can change the character of the PL from green to red, for example, in samples exposed to water by ageing and partially oxidizing the silicon, thus going through mostly hydrogen-terminated and/or nonterminated bonds to a partially oxidized Si structure. ${ }^{[409]}$ The light emission by a single point on the silicon surface may be monitored by a $3 \mathrm{D}$ reconstruction of the porous $\mu$-sized structure via $\mu$-CPL. The dots, which are clearly visible in $\mu$-CPL (Fig. 33(c)), are probably formed due to the use of non-equilibrium high-intensity ultrasonic surface treatment ${ }^{[413]}$ as a prospective method for surface modification. The positions of the dots are probably located on the spots where cavitation bubble collapse occurs along with a localization of a thermogradient on the surface ${ }^{[414]}$ during surface modification due to high intensity ultrasonication. 
It is promising to employ in situ methods in other areas and apply them to study of new aspects of the known photoreaction on semiconductor surface and in situ methods presented here are good examples.

\subsection{Microorganism response to photo-generated species}

The photo-produced ROS could alter the activity of microorganisms penetrating into an intracellular volume, which is interesting for both photo-destruction of bacteria ${ }^{[415]}$ and nanoscale machineries discussed here (Fig. 30a).

In particular, we address an example, where ROS photoproduced at $\mathrm{TiO}_{2}$ under illumination effectively induces prophages in the lysogenic bacteria to the lytic cycle. ${ }^{[416]}$ There was much discussion, how ROS affect microorganisms: whether they destroy the cell membrane or whether they affect nuclei of the cell or bacteria already before. ${ }^{[415]}$ The mentioned experiment with lysogenic lactic bacteria ${ }^{[416]}$ was planned in such a way not to deactivate or kill bacteria, but to use short term irradiation insufficient to kill bacteria to find out if ROS affect the deoxyribonucleic acid (DNA) of bacteria before their deactivation. In the case that ROS affect DNA stronger, they destroy the cell membrane via a prophage induction to the lytic cycle and cause release from the bacterium. From Fig. $\mathbf{3 4}$ it is evident, that photogenerated ROS are indeed released from a bacterium before it is deactivated, which means that DNA is affected faster than the cell membrane is destroyed.

It is also interesting to note that different microorganisms have different sensitivities to photogenerated species. At this point, an example can be drawn that gram-positive $(\mathrm{G}(+))-$ bacteria are more sensitive to superoxide anions $\left(\cdot \mathrm{O}_{2}^{-}\right)$in comparison with gram-negative $(\mathrm{G}(-))$ - bacteria. ${ }^{[383]}$ In particular, it was shown ${ }^{[383]}$ that the yield of the superoxide could be enhanced by the bimetallic $\mathrm{Ag} / \mathrm{Ni}$ nanoparticles deposited on $\mathrm{TiO}_{2}$ and $\mathrm{G}(+)$ bacteria being in general more stable to ROS because of an additional lipid membrane, by contrast to $\mathrm{G}(-)$, that are more sensitive to $\left(\cdot \mathrm{O}_{2}^{-}\right)$.

We want to highlight here that microorganisms can also be used for in situ detection of the formation of photochemical reaction products, which immediately changes their behavior on the surface. The fact that microorganisms are very sensitive to photoinitiated processes makes the proposed concept (see Fig. 30(a)) quite believable. This concept consists in guiding cell behavior on the surface. It would be extremely exciting if a spatiotemporal ion concentration gradient over the surface would guide cells locally promoting growth of one cell type (e.g., sulfur bacteria) and prevent the development of other cells to control biofilm formation and to detect selective biomolecules.

\section{Photocatalytic degradation of organic species on $\mathrm{TiO}_{2}$}

Recently there has been a tendency among photocatalyst investigations concerning photodecomposition of chemical ${ }^{[373]}$ and bacterial pollutants in an aqueous phase, ${ }^{[417]}$ that are focused on photocatalytically-active coatings ${ }^{[418]}$ capable to remove adsorbed organic contaminants under solar or artificial illumination (so-called self-cleaning materials ${ }^{[418]}$ ).

The self-cleaning characteristics of semiconductors result not only from their strong oxidation power under irradiation, but also from the discovered phenomenon of the photo-induced superhydrophilicity inherent in the surface. ${ }^{[419]}$ Thus one may consider the contribution of a self-cleaning function combined with stability of the organized soft matter on a semiconductor for novel "intelligent" materials and nanoscale machineries. 
When speaking about novel "intelligent" materials, the photocatalytic degradation of organic species can be mentioned as a negative process if one is interested in creating oscillating soft matter coatings ${ }^{[370]}$ on the semiconductor surface without polymer degradation during irradiation. The potential degradation of the organic substances and polymer matrix triggered by ROS should always take into account photocatalytic systems. ${ }^{[420]}$ The stability can be studied by means of a combination of spectroscopy with microscopy. ${ }^{[368]}$ For example, FTIR $^{[368,421]}$ has proved to be an effective method for the investigation of the composition before and after irradiation.

In the following, we will present examples using $\mathrm{TiO}_{2}$ with a low photoactivity and a lowintensity as well as short-term irradiation that allows for in situ modulation ${ }^{[368]}$ without degrading the polymer coating, in order to guide cell migration on the surface. ${ }^{[370]}$ This point is a promising route to in situ control the biofilm on the surface ${ }^{[382]}$ and to design new biosensors. ${ }^{[369]}$

\section{Prospects for in situ modulation of soft matter and microorganisms without degradation}

\subsection{Reversible oscillation of soft matter properties: thickness, stiffness, swelling}

Here, we study the transformation of electromagnetic energy into spatiotemporal ion concentration gradients, where the inorganic semiconductor material is a light sensitive system, and polyelectrolyte (PE) layer-by-layer ( $\mathrm{LbL}$ ) assembly. As a responsive element and with biofilm control, it is a prospective application for nanoscale machineries and biosensing. It should be noted that we primarily aim here on the in situ characterization of changes in the material, formation of self-assembly, soft matter modulation, and light sensitive particle vortices.

In Fig. 35, several methods are highlighted for in situ MS studies. Microchips covered with semiconductor and soft matter can be designed as microfluidic probe that allows real-time imaging of the electrode-liquid electrolyte interface (Fig. 35(a)). ${ }^{[422]}$ The same chips could be applied in an open cell for in situ quartz crystal microbalance (QCM) studies to follow both LbL assembly and photoactivation of the films (Fig. 35(c)). ${ }^{[423]}$ Photoactivation of the film can also be studied with the help of atomic force microscopy (AFM) (Fig. 35(d)) with time resolution and localized at a certain area.

Recently, a specific LbL architecture for advanced bioapplications has been introduced: reversible oscillation of soft matter on semiconductor surface, ${ }^{[368]}$ resulting in cell migration, ${ }^{[370]}$ desorption from the surface, ${ }^{[382]}$ and modulation of $\mathrm{pH}$ sensitive chemical bonds in LbL assemblies, ${ }^{[369]}$ and drug chemicals delivery. ${ }^{[420,421,423,424]}$

It is important to mention the high priority to monitor soft matter response on the surfaces (Fig. 30) measuring charge separation, ion and ROS generation and localization, activation and relaxation time, process reversibility as well as trapping of ions and molecules in the dependence of various parameters, such as thickness, stiffness, roughness, hydrophilicity, and permeability. It is of high priority to obtain knowledge about the behavior of ion-enriched materials in nanoconfinement. Information about the stability of ion-enriched soft matter inside nanoconfined volumes and the interaction between different ion enriched reagents and ion migration will be obtained during further examinations. Knowledge of how to organize LbL assemblies with different ion content, e.g., for different protonation, as well as of the assembly from different $\mathrm{pH}$ and ionic strength solutions for the formation of multifunctional gradient material with different affinity to store and exchange photogenerated ions is 
important. Systematic studies of the mechanism of film formation will be instructive in order to control biofilms on the surface.

\subsection{Bioresponse due to soft matter oscillation and photoreactions}

It has already been mentioned above and shown in Fig. 36, that some very specific "intelligent" systems were developed recently ${ }^{[370,382]}$ to control system bioactivity, but unfortunately no general concept of biofilm regulation has been suggested yet. Reversible oscillation of LbL films based on high amplitude actuation of block copolymer micelles (BCM) allows controlling cell behavior on a surface. In particular, change of stiffness results in migration of osteoblasts to a harder part of the surface (Fig. 36(a,b) $)^{[370,382]}$ and in desorption of bacteria from the surface with time (Fig. 36(c)). ${ }^{[382]}$

Andreeva et al. ${ }^{[369]}$ suggested a new principle of photo-assisted spatial desorption of (poly)histidine-tagged (His-Tag) proteins on a $\mathrm{TiO}_{2}$ surface (Fig. 37). Here, a semiconductor $\mathrm{TiO}_{2}$ surface is decorated by an LbL assembly of a strong polyelectrolyte, namely, polystyrenesulfonate (PSS), and nickel-nitrilotriacetic acid (NTA). The PSS/NTA multilayer architecture provides n-fold (nxNTA) binding efficiency for more precise protein recognition in comparison to existing molecular His-Tag protein recognition with one-and-threefold multiplication. Spatially resolved desorption of proteins is regulated by non photodestructive short-term low-intensity light irradiation. The local $\mathrm{pH}$ shift on irradiated $\mathrm{TiO}_{2}$ selectively affects the $\mathrm{pH}$-sensitive NTA/protein complex, but not the LbL assembly of PSS and NTA, which is stable in a broad $\mathrm{pH}$ range. It can be noted that in situ characterization of LbL organization and its following in situ modulation can be monitored by SIET, $\mu$-CFM and QCM (Fig. 37). ${ }^{[369]}$

The examples discussed above clearly demonstrate that microorganisms are sensitive to both products of photoreactions, e.g., ROS, $\mathrm{pH}$ change in the system, and actuation of soft matter. This strongly supports our suggested concept to build a nanoscale machinery for controllable manipulation of microorganisms with biomolecules on a semiconductor surface. A systematic study of the design of such a nanoscale machinery is needed to modulate a pronounced bioresponse, to build a general concept and to integrate the system into microfluidic chips or microbial biosensors. To have pronounced modulation of soft matter, different ion, e.g., proton, gradients need to be studied on surfaces in detailed manner.

One more important topic is the spatiotemporal delivery of ions for modern synthetic biology platforms (biofactories). The development of alternative, green transformation of electromagnetic energy into ion gradients and non-petrochemical feedstocks requires a new vision on industrial bioreactors, because more and more complex compounds (proteins, bacteria) are used in production now or will be used in the future. This calls for more precise control over the reactions occurring in bioreactors. Our systems for manipulation of biomolecules and bacteria provide controlled release of the necessary ions for bioobject activation exactly at the required bioreaction stage and place, which may cause a considerable biotechnology breakthrough in the future.

\subsection{Chemicals delivery: regulation of soft matter permeability and motion}

An advantage of many microbial biosensors is that the immobilization of whole cells improves the stability of intracellular biorecognition elements, such as enzymes, because the enzyme is retained in the natural environment; moreover, the employment of genetically engineered microorganisms permits on a drastic improvement of the sensitivity and selectivity of the resultant microbial biosensing devices. In order to integrate the microbial biorecognition elements into lab-on-chip devices, one needs to exert a remote control over 
adsorption and desorption of microbial cells as well as over their biochemistry, e.g., by delivering different ions. To this end, the conformational transitions in the PE films induced by photocatalytic reactions occurring at the semiconductor surface in contact with the polyelectrolyte can be used. Thus, for example, in our previous works, we discussed the possibility of reversible photo-opening ${ }^{[425]}$ of polyelectrolyte assemblies to affect the adsorbed microorganisms. ${ }^{[426]}$ Indeed, self-oscillation of LbL films of different architectures provides a high gain for various bio-applications from protein and pathogenic cell recognition to cell detachment, migration and construction of lab-on-chip elements. However, the development of a general concept for process regulation on "demand" is challenging-but possible and rewarding as well.

A prominent example of the photocatalytically-active titania-based LbL coatings is shown in Fig. 38(a). ${ }^{[421]}$ The coatings were obtained by the immobilization of loaded titania/PEs nanoparticles into a sol-gel-derived matrix. By forming a PE shell on the mesoporous titania particles, it is possible to fabricate nanoscale reservoirs, which can store relatively large organic molecules in their interior. The mesoporous titania particles were loaded with the luminescent dye Rhodamine 6G before the deposition of the PEs shell, and the light-driven release has been shown. Properties of biomolecule-containing LbL films, such as their growth regime, thickness, loading capacity, and mobility of polymers within the film, can be altered and synthetic polymers or biopolymers can constitute the PE film.

Two types of pH-responsive encapsulation systems based on surface metal sponges prepared by sonochemical treatment were proposed: (i) a single-step system with a simultaneous activation and modification of the metal and active compound by HIUS, and (ii) a system where the metal sponge serves for the construction of PE surface capsules, allowing storage and release of an encapsulated substance. ${ }^{[426]}$ In the first system, the encapsulation is ensured via the chemisorption of the active compound on the surface, i.e., $-\mathrm{OH}$ groups of the metal oxide layer. In contrast, in the case of PE surface capsules there is no need of specific interactions between a metal and the encapsulated compound, and therefore, it is a universal encapsulation and carrier system. Moreover, hybrid systems with polypyrrole are developed, providing an efficient delivery and step-wise release of the low molecular weight active component. ${ }^{[425]}$ Further examples have been analyzed in recent reviews, ${ }^{[366,367,380]}$ proving that the strategy discussed is suitable to build probably even a light sensitive autonomous robot $^{[427]}$ for drug and cell delivery.

Photocatalytic reactions recently were used more and more for development of (nano-)tools for autonomous and remotely guided catalytically self-propelled motion, as for example shown in Fig. 38(b) for InGaAs/GaAs/(Cr)Pt tubes. ${ }^{[428]}$ The rolled-up tubes with diameters in the range of 280-600 $\mathrm{nm}$ move in hydrogen peroxide solutions with speeds as high as $180 \mu \mathrm{m}$ $\mathrm{s}^{-1}$. The effective transfer of chemical energy into translational motion has allowed these tubes to perform useful tasks such as transport of cargo.

Janus photocatalytic micromotors hold considerable promise for diverse practical applications. A highly efficient light-driven photocatalytic $\mathrm{TiO}_{2}-\mathrm{Au}$ Janus micromotor with wireless steering and velocity control is shown in Fig. 38(c). ${ }^{[29]}$ Unlike chemically propelled micromotors, which commonly require the addition of surfactants or toxic chemical fuels, the fuel-free Janus micromotor (diameter $\sim 1.0 \mu \mathrm{m}$ ) can be powered in pure water under an extremely low ultraviolet light intensity $\left(2.5 \times 10^{-3} \mathrm{~W} / \mathrm{cm}^{2}\right)$, and with $40 \times 10^{-3} \mathrm{~W} / \mathrm{cm}^{2}$ they can reach a high speed of 25 body length/s, which is comparable to common Pt-based chemically induced self-electrophoretic Janus micromotors. 
The concept of a surface combustion microengine is demonstrated on a microcantilever covered with a thin $\mathrm{TiO}_{2}$ layer (Fig. 38(d,e)). ${ }^{[428]}$ Irradiation of this microengine produces controlled bending of the microcantilever as a result of differential stress produced by photocatalytic oxidation of organic molecules on the $\mathrm{TiO}_{2}$ coating. Surface combustion based microengines would require less maintenance in minimally controlled field environment and could be potentially used in construction of miniature movable machines and conversion of solar energy to mechanical work, when extended to a large array of microcantilevers.

There is much attention nowadays to the development of light driven, ${ }^{[430]}$ autonomous ${ }^{[431]}$ soft robots (Fig. 38(f)), and we suggest photocatalytic fuel production as an on-board fuel supply. Gas that may be generated from the fuel photodecomposition inflates fluidic networks downstream of the reaction sites, resulting in actuation. 


\section{Conclusion}

After Herbert Krömer's statement in his Nobel lecture in $2000,{ }^{[1]}$ today we can even more strongly note that the interface is still the device. ${ }^{[432]}$ The increase in complexity is attended by the involvement of interfacial chemistry, different chemistry environments, liquids, and interfaces under operation in non-inert ambient conditions. In this review, we have highlighted a variety of in situ spectroscopy routes to analyze the formation of surfaces and buried interfaces on the atomic scale in complex chemical surroundings during growth and preparation as well as during operation. On the example of several material systems such as III/V semiconductors, oxides, silicon, germanium, organic molecules, biomaterials, and hybrids of these materials as well as various examples of application with photo-induced reactions, we have shown that there are opportunities to scrutinize nucleation of materials, interface formation, growth as well as more complex interfacial reactions.

Starting from clear and well-defined epitaxial interfaces in vapor phase ambient, such as InP and GaP (100) surfaces, which are on the one hand highly relevant for record-breaking solar energy conversion and which on the other hand act as types of "drosophila" of atomic scale in situ control, we afterwards illustrate their modification and chemical functionalization via different adsorbants, hetero contacts and solid-liquid interfaces, involving industrially relevant and opto-electronic generic technology developments such as the growth of III/V compounds on silicon. These formation scenarios have been thoroughly studied by optical in situ spectroscopy (i.e. RAS) and benchmarked by a broad spectrum of relevance to the interfacial science. High-efficiency water splitting devices were achieved by an in situ controlled interface functionalization of well characterized surfaces of epitaxial semicondcutor heterostructures. As a next step of surface functionalization, photochemical reactions at hybrid interfaces have been monitored in situ such as the detection of ROS, measured with specific ROS scavenging agents. Proton photogeneration has been studied with scanning probe in situ techniques such as scanning vibrating electrode technique, imaging mass spectrometry, and scanning ion-selective electrode.

However, there is still a huge challenge and a large and deep gap to be closed in the near and far future between the atomic scale understanding of model surfaces and interfaces, such as epitaxially prepared (100) surfaces, on the one hand and transient interfacial reactions under operation, such as in electrochemistry or ultrafast dynamics, on the other hand. There are many open tasks and questions that can be addressed by routes shown in this review. In photo-electrochemistry, solid-liquid interfaces are huge challenges with regard to efficient functionality, stability/corrosion and catalysis. In situ spectroscopy, such as near-ambient pressures photoelectron spectroscopies (APXPS) or RAS, will largely contribute to a microscopic understanding of the solid-liquid interface formation and functionalization. The key will be a detailed understanding of model systems and subsequent gradual lifting of the model character towards realistic application scenarios. In bio-applications, critical and conflicting requirements have to be addressed, such as having a sufficient defect concentration in the semiconductor to increase visible light absorption without limiting the carrier mobility too much via defect sites deep in the band gap. For spatial separation of oxidation versus reduction, the carrier mobility should be very different for electrons and holes and on the organic side of biosensing, there is a competition between sensitivity, buffering, and stability. Also here, we believe that device design for such demanding applications strongly benefits from a detailed understanding of complex interfacial reactions, which in turn is best achieved by understanding the relevant processes starting from relatively easy model interfaces with increasing complexity to the final device structure. Thereby, the more complex the interface formation gets, the more important in situ characterization and in 
situ control over the involved processing steps become. Efforts for in situ characterization must be increased, in particular, combining spectroscopic and scanning probe techniques.

\section{Acknowledgements}

The authors would like to express their gratitude to Prof. Dr. W.G. Schmidt for providing the theoretical data plotted in Fig. 7, to Dr. A. Dobrich and Dr. U. Seidel for providing the data for Fig. 4, to Dr. U. Seidel for the RAS data plotted in Fig. 10, to Dr. P. Kleinschmidt for the sketches shown in Fig. 14, as well as to Dr. P. Kleinschmidt, J. Luzcak and A. Nägelein for the STM images shown. We are indebted to Dr. O. Romanyuk, Prof. Dr. W. Daum, Dr. G. Lilienkamp, and Dr. B. Borkenhagen for fruitful scientific discussions regarding $\mathrm{GaP} / \mathrm{Si}(100)$ interfaces, as well as to Prof. Dr. H. Möhwald, and Prof. Dr. P. Fratzl regarding prospects and challenges of regulation of processes at inorganic / polymeric / biological interfaces. This work was financially supported by the German Research Association (DFG, proj. no. HA3096/4-2) and the German National Academy of Sciences Leopoldina (MMM, grant no. LPDS 2015-09).

Received: ((will be filled in by the editorial staff))

Revised: ((will be filled in by the editorial staff)) Published online: ((will be filled in by the editorial staff)) 


\section{Figures and captions}

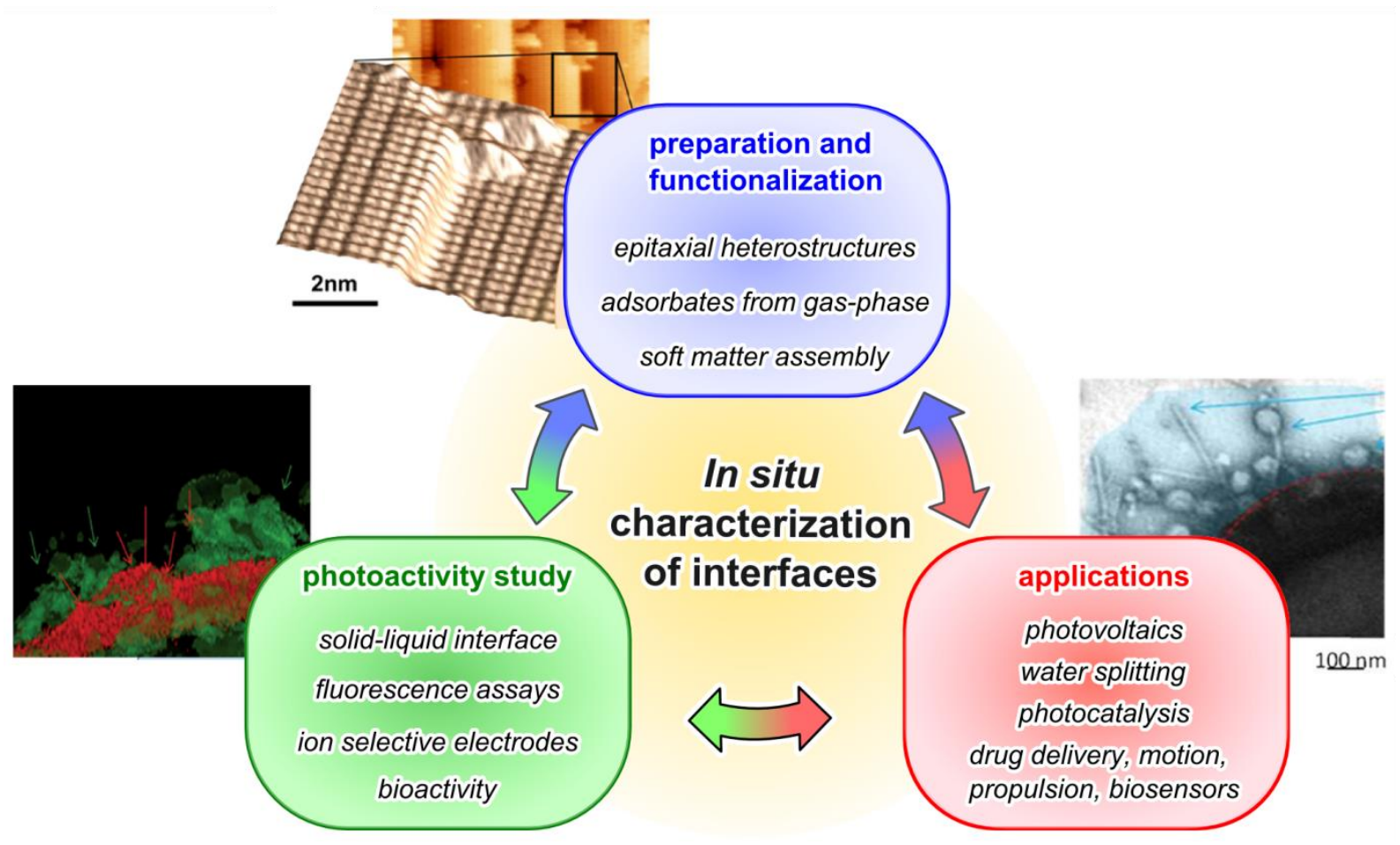

Figure 1. In this review, we focus on in situ studies on interfaces of materials, which are relevant for application in energy conversion and biosensing. We focus on in situ characterization during non-UHV preparation techniques and photoactivity aiming at control over interfacial reactions in real time. 


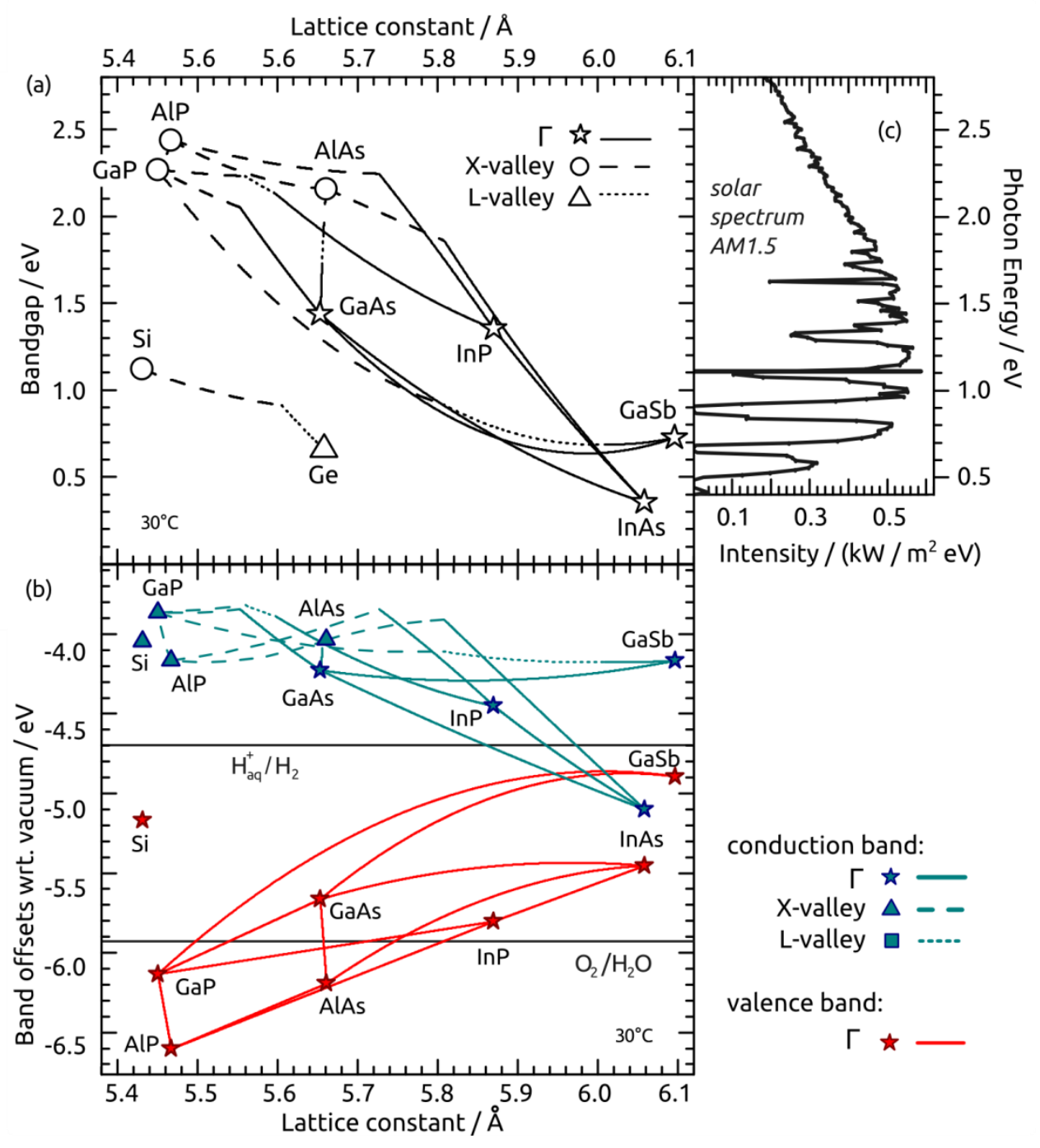

Figure 2. (a) Band gaps of binary and ternary III/V compound semiconductors (line and symbol style indicate the nature of the bandgap), data from Ref. ${ }^{[433]}$; (b) Approximated conduction (turquois) and valence band (red) offsets (line and symbol style indicate the nature of the bandgap), data from Ref.s ${ }^{[20,433,434,435,436]}$; adapted from Ref. ${ }^{[20]}$; (c) Solar spectrum AM1.5 ASTM-G-173-03 from Ref. ${ }^{[437]}$ 
Theoretical efficiency limit of dual-junction absorbers (current-matched detailed balance, AM1.5g) maximum efficiency / \%

51015202530354045 (a) PV efficiency, no thinning

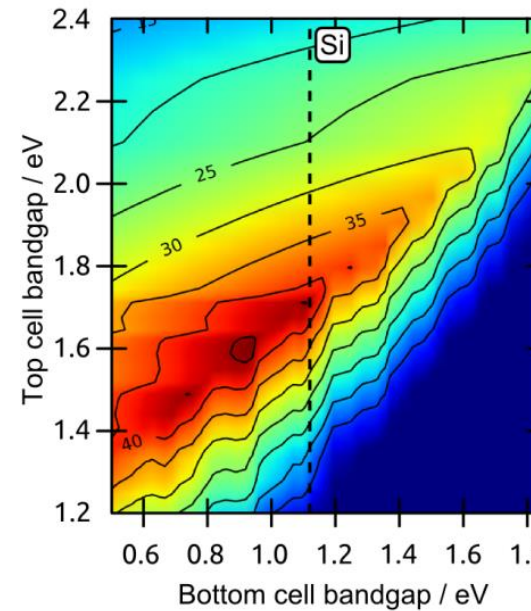

(b) PV efficiency, thinning

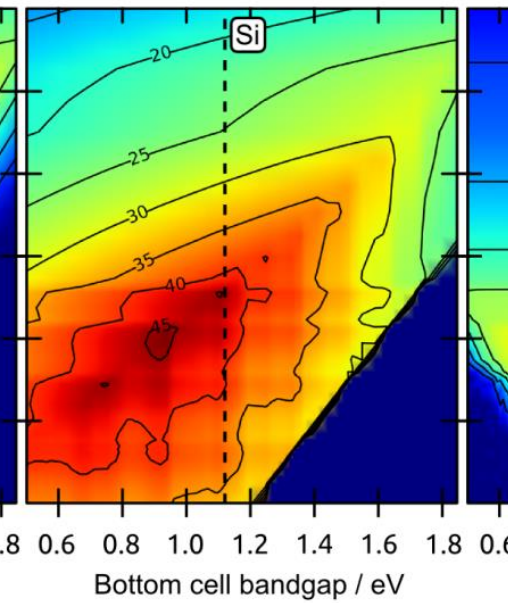

(c) STH efficiency, thinning

Figure 3. Theoretical efficiency limit for a two-junction absorber structure in the currentmatched detailed balance limit operated as $(a, b)$ a photovoltaic cell (electric efficiency) and (c) a water splitting device (solar-to-hydrogen efficiency) in dependence of the bandgap of the

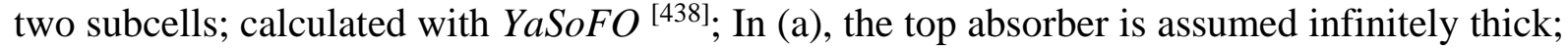
In $(b, c)$, the thickness of the top absorber is reduced to optimize current-matching with the bottom cell; The bandgap of $\mathrm{Si}$ is marked for the bottom cell and the inset on the right indicates the layer structures for a corresponding III/V-on-Si tandem cell with a GaP nucleation layer; for the water splitting device, a water layer of $1 \mathrm{~mm}$ thickness and an $\mathrm{IrO}_{2}$ counter-electrode with $1 \mathrm{Ohm}$ solution resistance were assumed. 


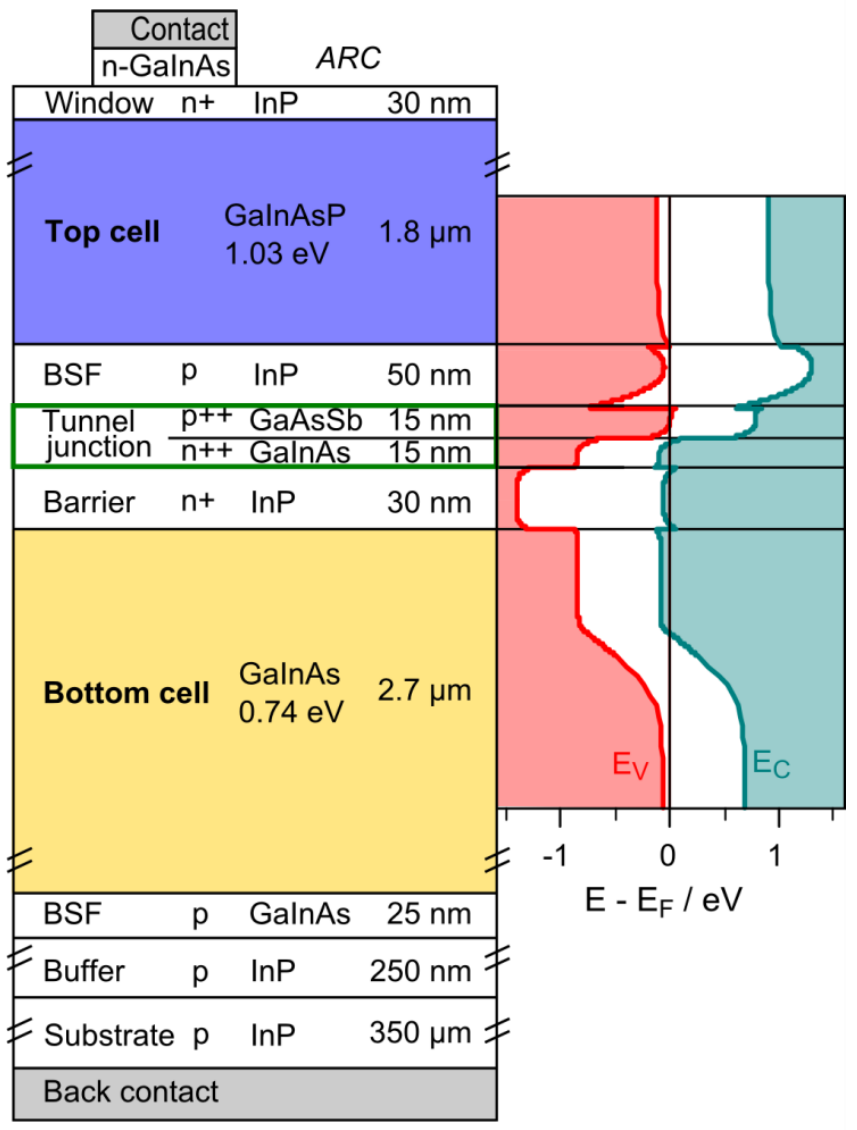

Figure 4. (left) Schematic of the stack and (right) approximated band diagram for an InPbased tandem solar cell optimized for absorption in the infrared as lower tandem in a four junction solar cell $\left(\mathrm{BSF}=\right.$ back surface field); adapted from Ref. $\mathrm{s}^{[28,22]}$ 


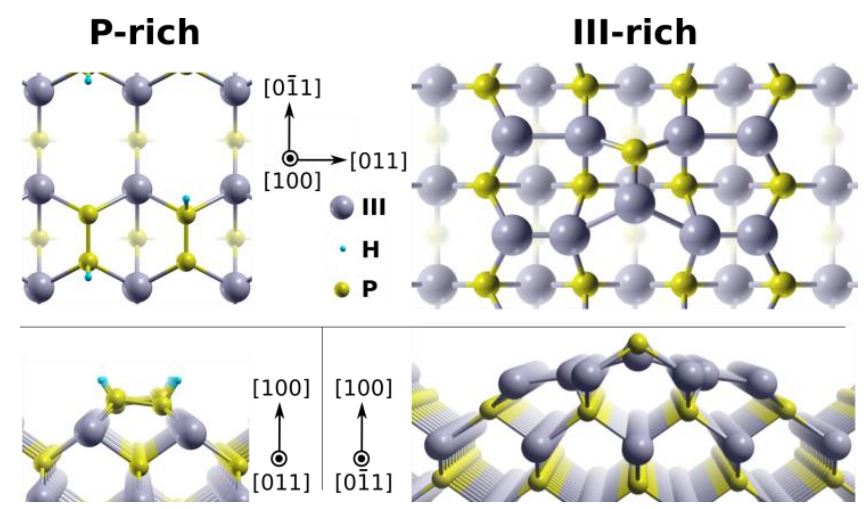

Figure 5. Surface reconstructions of (left) P-rich and (right) group-III-rich InP resp. GaP (100) surfaces. The top row shows the top view and the bottom row side views in [0-11] and [011] direction, respectively. Adapted from Ref.[50]. 


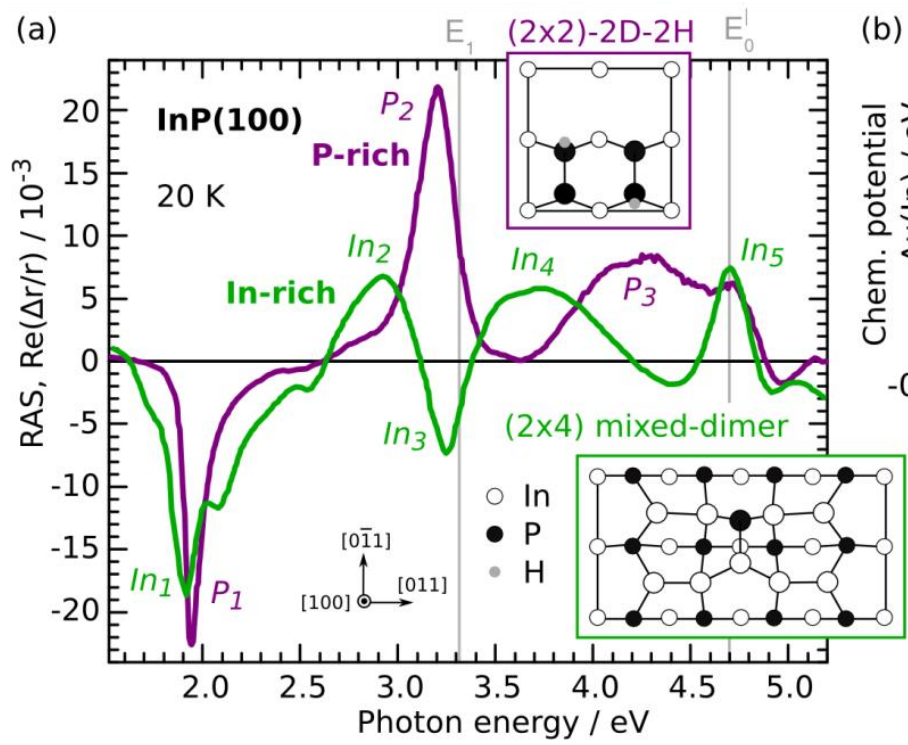

(b)

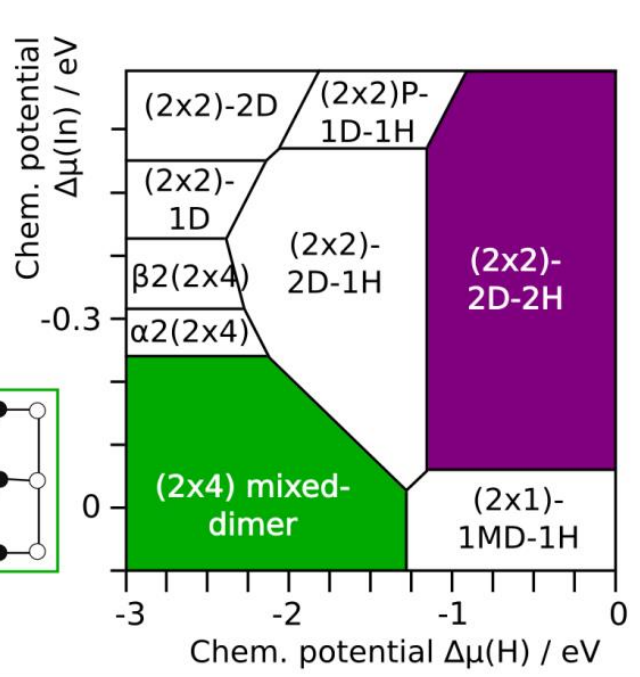

Figure 6. (a) RAS of P-rich, (2x2)-reconstructed (violet) and In-rich, (2x4)-reconstructed (green) $\mathrm{InP}(100)$ surfaces, measured at $20 \mathrm{~K}$, data from Ref ${ }^{[38]}$; the insets show ball-and-stick models of the corresponding surface reconstruction; gray vertical lines indicate the critical interband transitions of $\mathrm{InP}^{[439]}$; (b) phase diagram in dependence of the In and $\mathrm{H}$ chemical potential, data from Ref. ${ }^{[32]}$ 


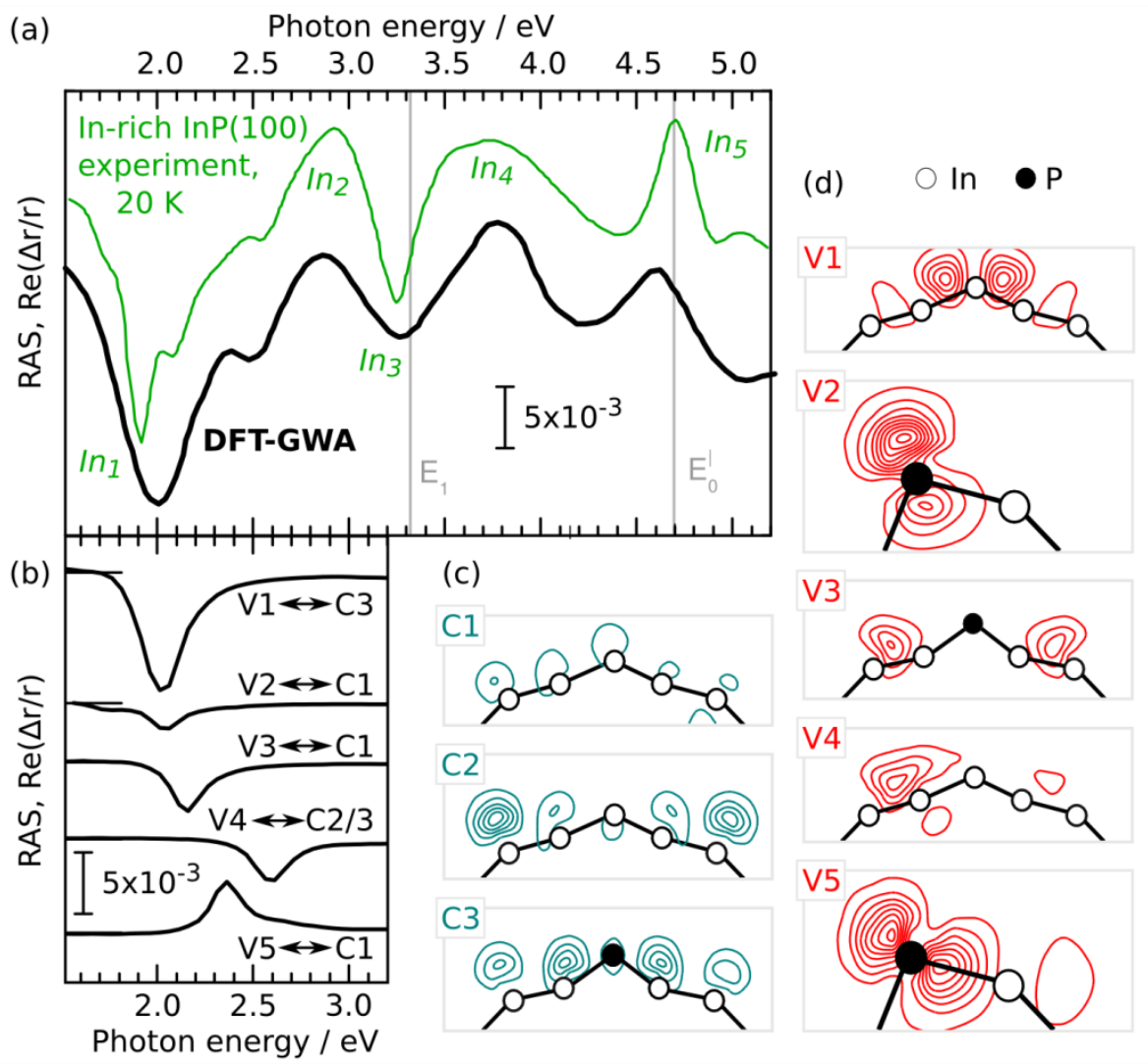

Figure 7. RAS of In-rich InP(100): (a) comparison of experiment (green) and DFT-GWA calculation (black); (b) contributions to the calculated RA spectrum from different transitions involving the surface states in the conduction (c) and valence band (d); gray vertical lines indicate the critical interband transitions of $\mathrm{InP}^{[439]}$; adapted from Ref. ${ }^{[36]}$, data from Ref. $\mathrm{s}^{[36 \text {, }}$ 38] 


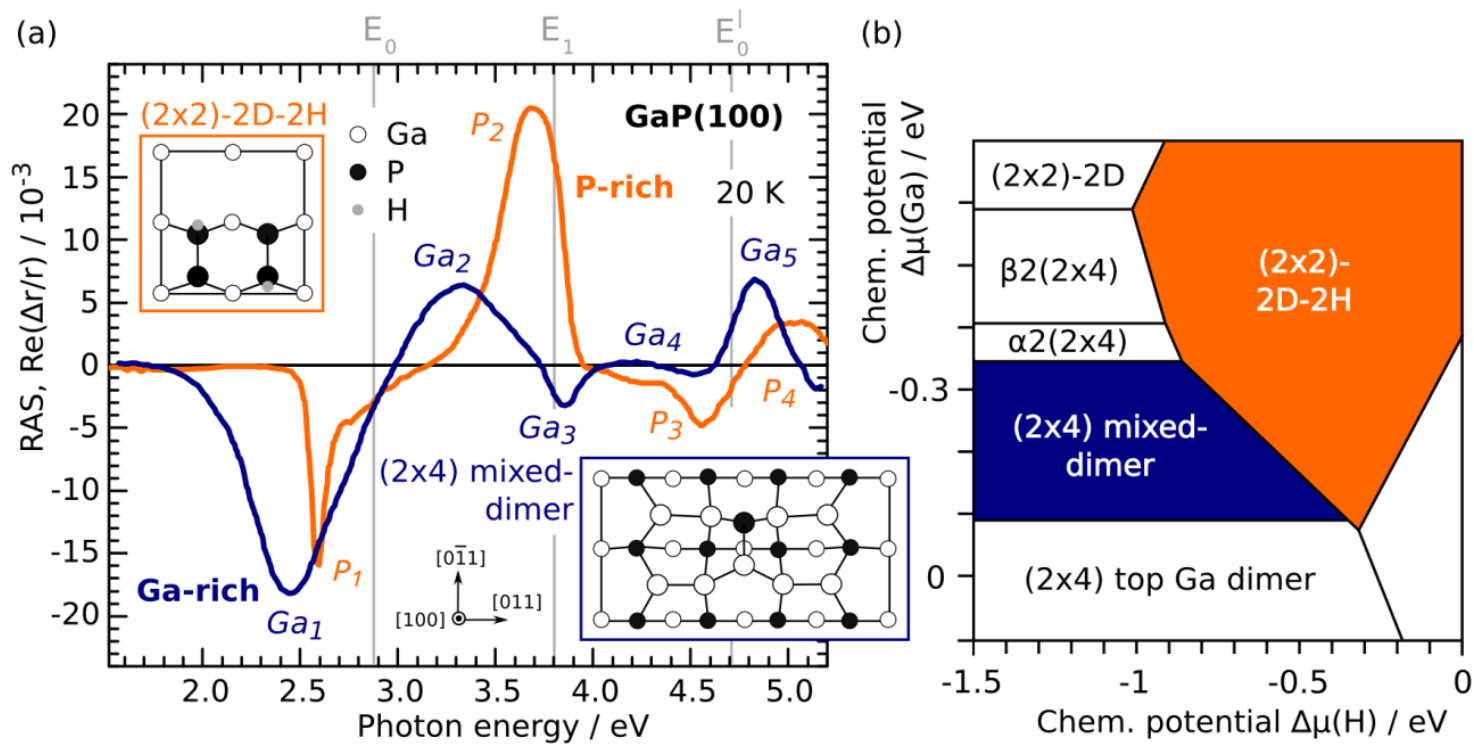

Figure 8. (a) RAS of P-rich, (2x2)-reconstructed (orange) and Ga-rich, (2x4)-reconstructed (blue) $\mathrm{GaP}(100)$ surfaces, measured at $20 \mathrm{~K}$, data from Ref. ${ }^{[63]}$; the insets show ball-and-stick models of the corresponding surface reconstruction; gray vertical lines indicate the critical interband transitions of $\mathrm{GaP}^{[440]}$ (b) Phase diagram in dependence of the $\mathrm{Ga}$ and $\mathrm{H}$ chemical potential, data from Ref. ${ }^{[66]}$ 

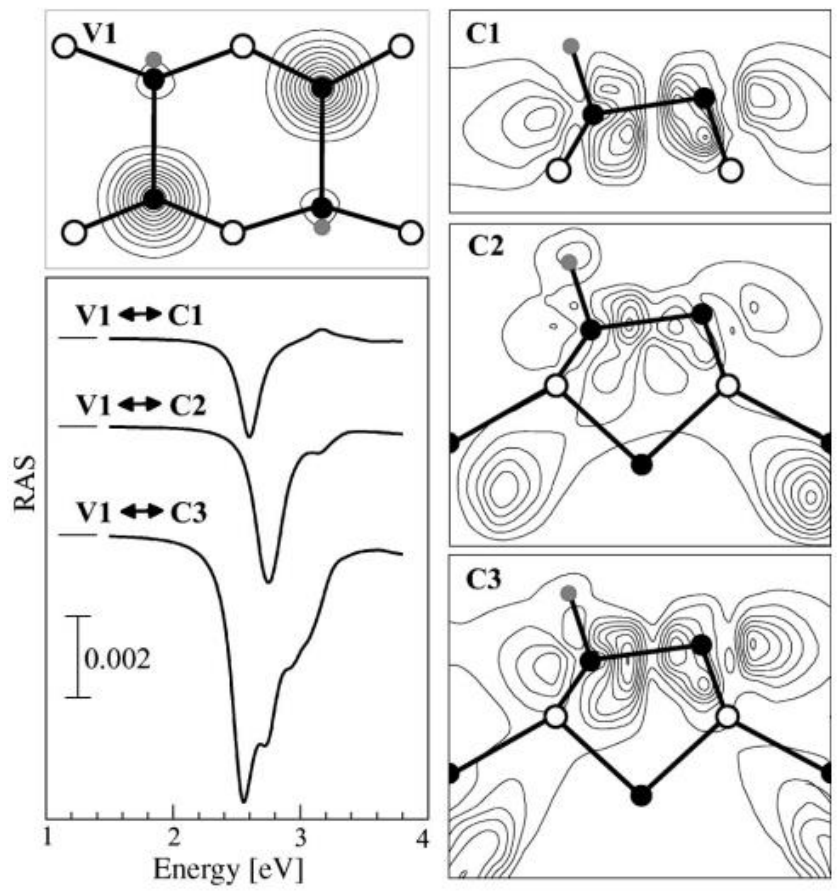

Figure 9. Orbital character of specific surface states of the P-rich, $(2 \times 2)$ reconstructed $\mathrm{GaP}(100)$ surface (at the $\mathrm{K}$ point of the Brillouin zone) and contributions of transitions between these states to peak $P 1$ in the RA spectrum of the P-rich, $(2 \times 2)$ reconstructed $\mathrm{GaP}(100)$ surface as calculated by DFT-GWA; Reproduced with permission. ${ }^{[66]}$ Copyright 2003 The American Physical Society. 


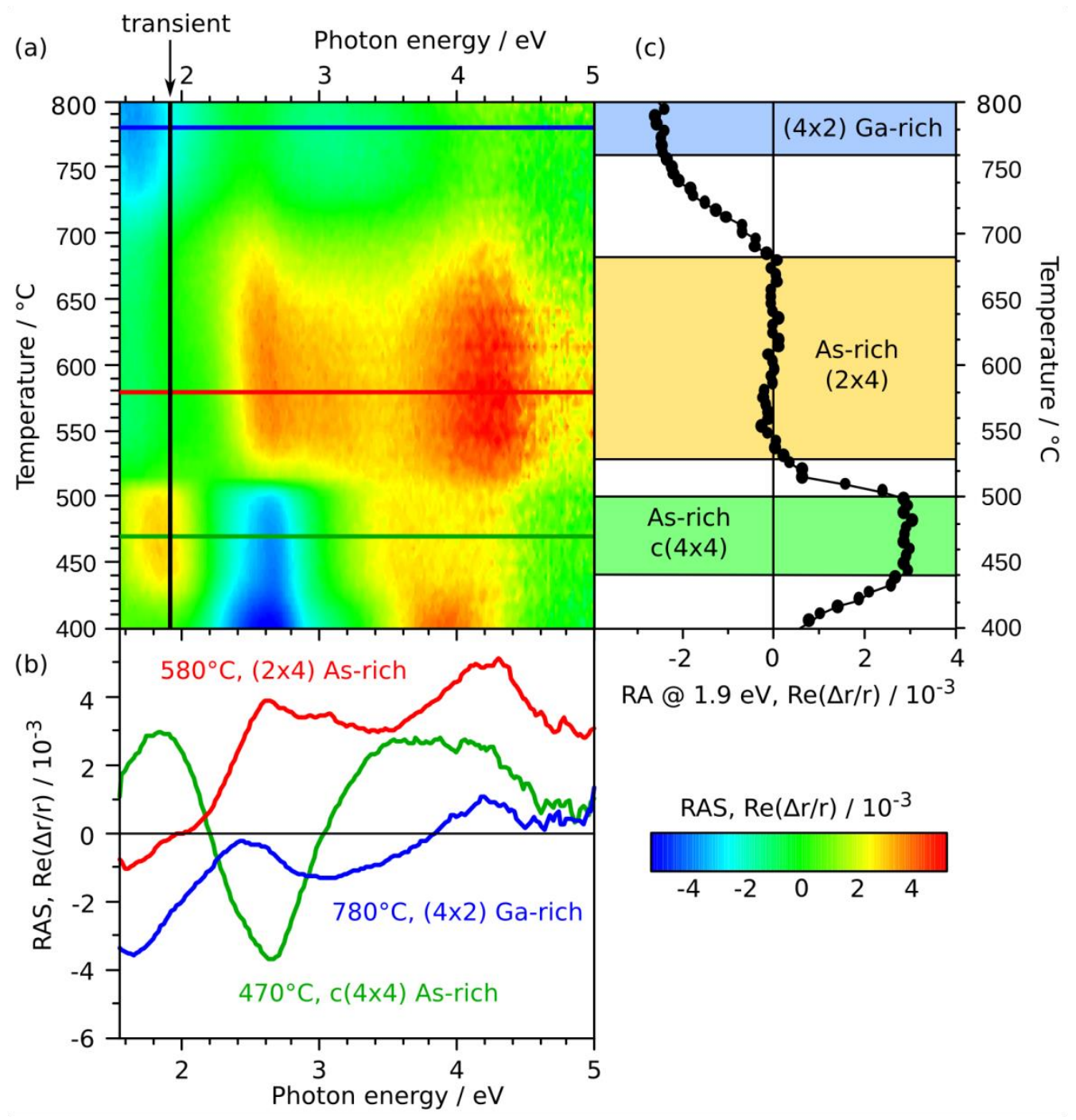

Figure 10. (a) Color-coded RA spectra of GaAs(100) during annealing in $\mathrm{H}_{2}$ ambient, where lines mark spectra extracted in (b): With increasing temperature, the As-rich $c(4 \times 4)$ surface (green) transforms to the As-rich $(2 \times 4)$ surface (red) and finally to the Ga-rich $(4 \times 2)$ surface (blue). Despite the changing temperature (which causes shifts in the RAS signal), the surface formation can be followed in situ with a transient at $1.9 \mathrm{eV}$ (c); data obtained by Dr. U. Seidel (formerly Hahn Meitner Institute, Berlin, Germany). 


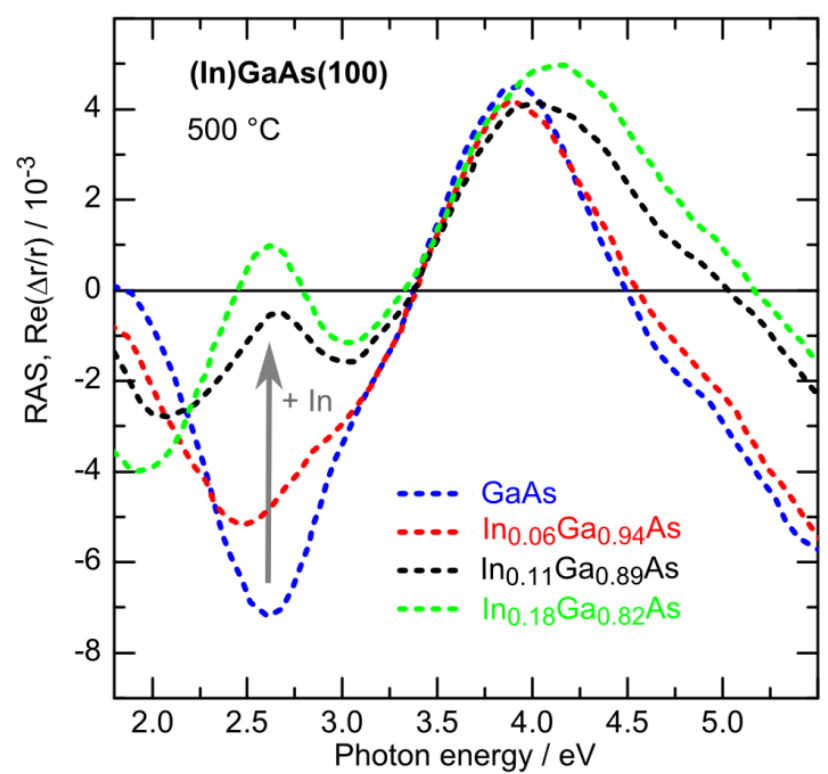

Figure 11. RA spectra of $\operatorname{GaAs}(100)$ and $\operatorname{InGaAs}(100)$ measured at $500{ }^{\circ} \mathrm{C}$. The lineshape of the spectra depends on the stoichiometry; data from Ref. ${ }^{[127]}$. 


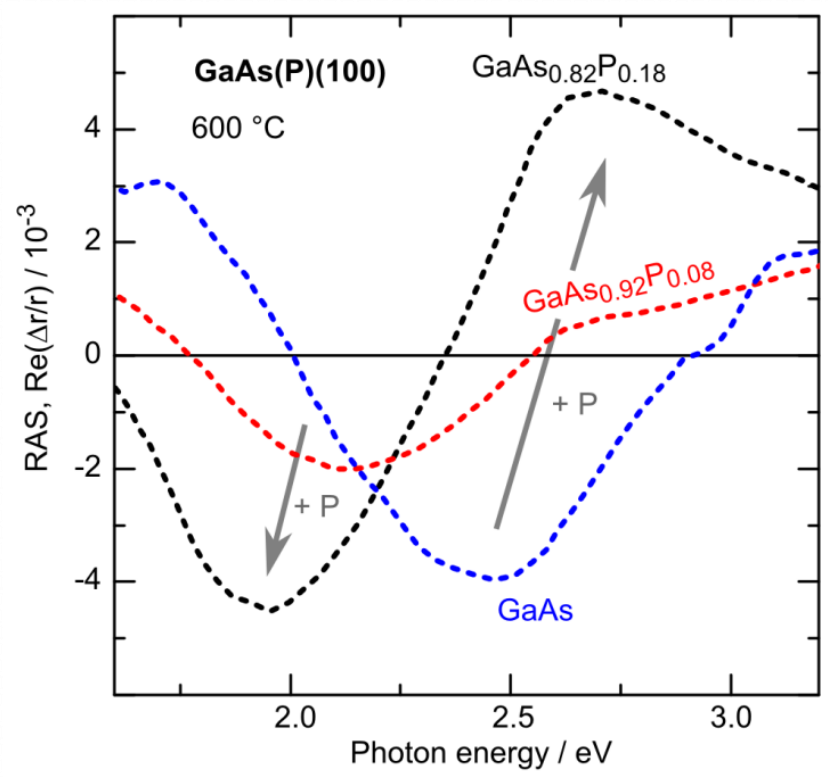

Figure 12. RA spectra of $\operatorname{GaAs}(100)$ and $\mathrm{GaAsP}(100)$ measured at $600{ }^{\circ} \mathrm{C}$. The lineshape of the spectra depends on the stoichiometry; data from Ref. ${ }^{[127]}$. 


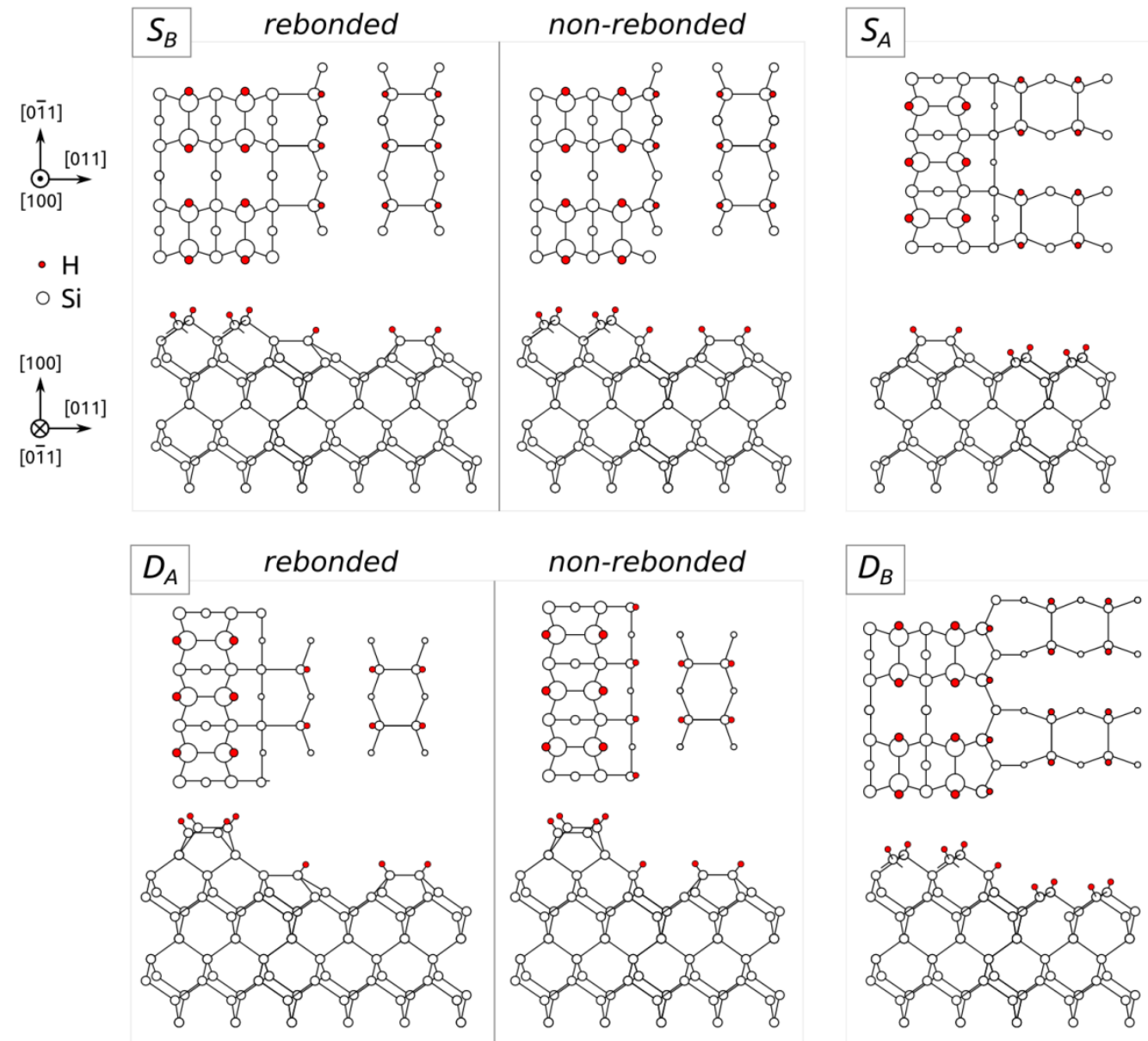

Figure 13. Single (top panel) and double-layer (bottom panel) step configuration at the monohydride-terminated $\mathrm{Si}(100)$ surface, shown in top view (first line in each panel) and side view (second line in each panel). At single-layer step edges $S$ (or odd multiples), dimer rows on adjacent terraces are mutually perpendicular, while they are parallel across double-layer step edges $D$ (or even multiples). The index $A$ or $B$ indicates whether the dimer rows on the upper terraces are aligned in parallel or perpendicular to the step edges, respectively. For $S_{B}$ and $D_{A}$ steps, also the rebonded configuration is shown; modified after Ref. ${ }^{[157]}$ 


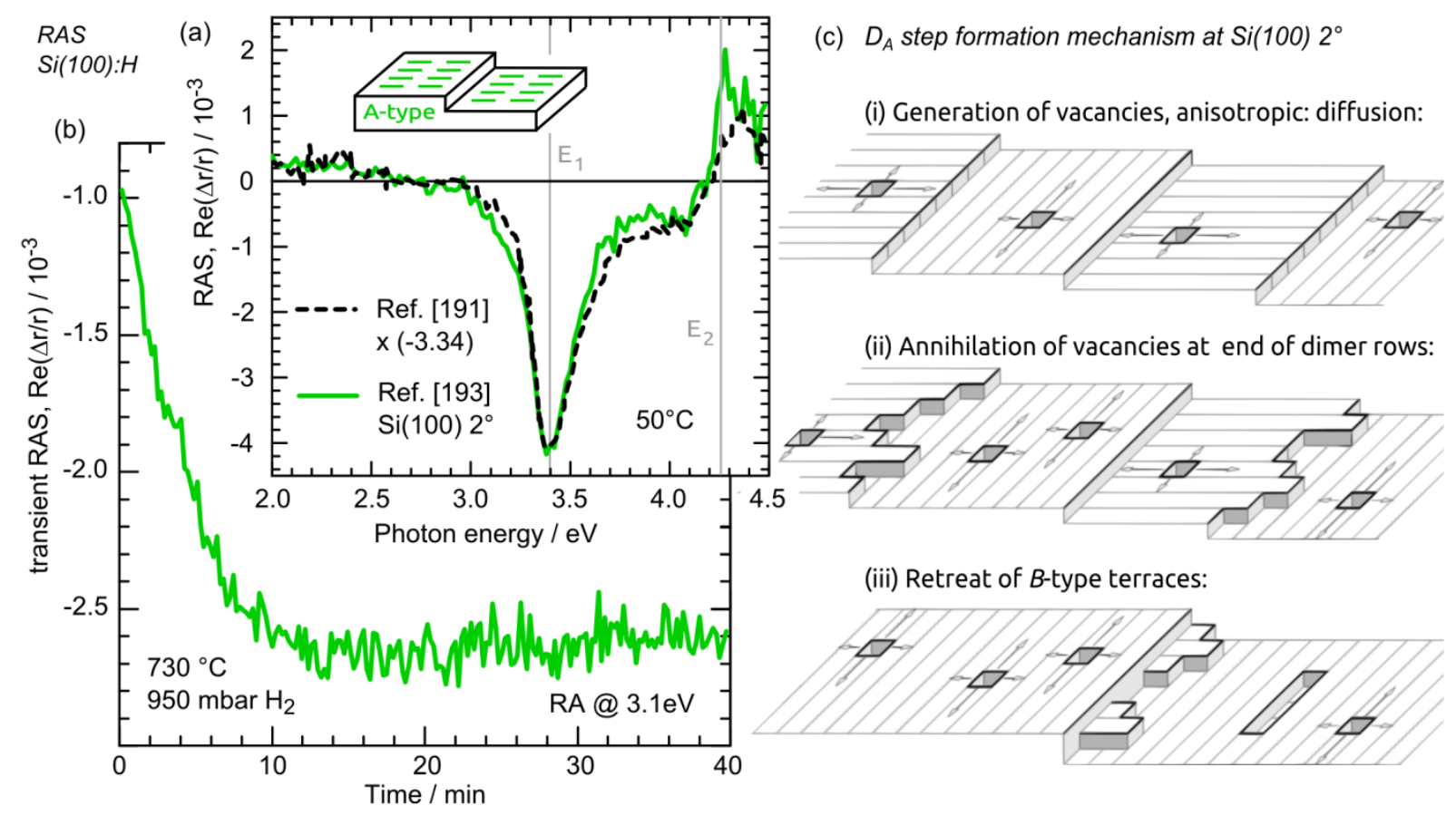

Figure 14. (a) RA spectrum of a (1x2) reconstructed monohydride-terminated $\mathrm{Si}(100)$ surface with $2^{\circ}$ misorientation towards [011] (about 85\% A-type domains) from Ref. ${ }^{[193]}$ (green line, measured at $50{ }^{\circ} \mathrm{C}$ ) compared to a flipped and scaled B-type surface from Ref. ${ }^{[191]}$ (A:B = 40:60), , (b) time-resolved in situ RAS at the $\mathrm{E}_{1}$ interband transition during annealing of $\mathrm{Si}(100) 2^{\circ} \rightarrow$ [011] in $950 \mathrm{mbar} \mathrm{H}_{2}$ at $730{ }^{\circ} \mathrm{C}$, (c) schematic of the anomalous $D_{A}$ step formation on $\mathrm{Si}(100) 2^{\circ} \rightarrow$ [011] surfaces $\mathrm{Si}$ vacancy generation and diffusion ${ }^{[193]}$; vertical gray lines indicate the interband transitions of $\mathrm{Si}^{[441]}$ 


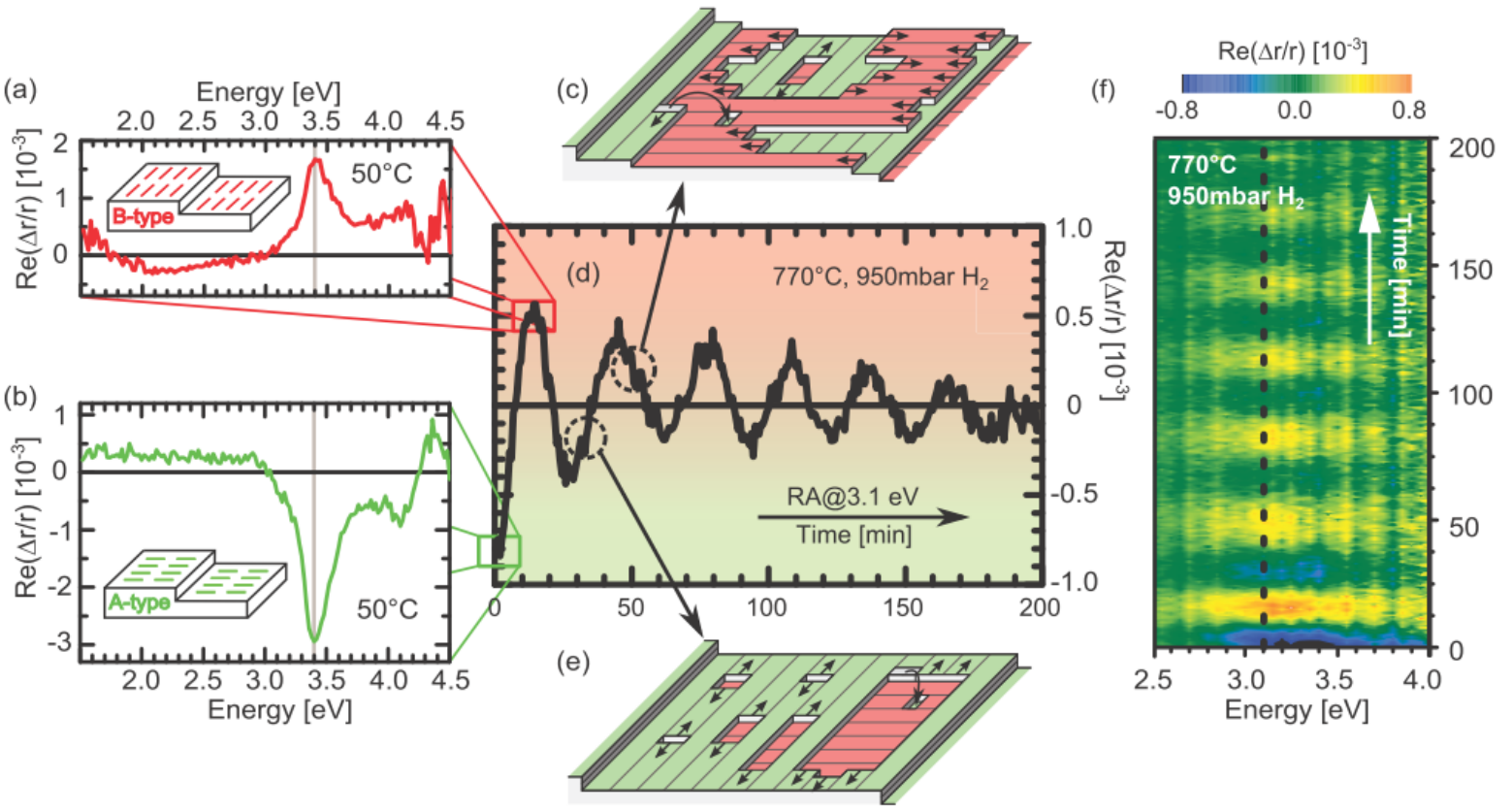

Figure 15. In situ RAS of almost exactly oriented $\mathrm{Si}(100)$ with $0.1^{\circ}$ misorientation towards [011]: spectra of majority B-type (a) and A-type domains (b) after preparation in $\mathrm{H}_{2}$ ambient (measured at $50{ }^{\circ} \mathrm{C}$ ). (f) Continuously measured RAS during annealing at $770{ }^{\circ} \mathrm{C}$ in $950 \mathrm{mbar}$ $\mathrm{H}_{2}$ (41 s/spectrum). An extracted RAS transient at the $\mathrm{Si} \mathrm{E}_{1}$ interband transition is shown in (d). The schematics in (c) and (e) indicate the vacancy formation causing the layer-by-layer removal process; Reproduced with permission. ${ }^{[196]}$ Copyright 2013, CC BY 3.0, S. Brückner et al. 


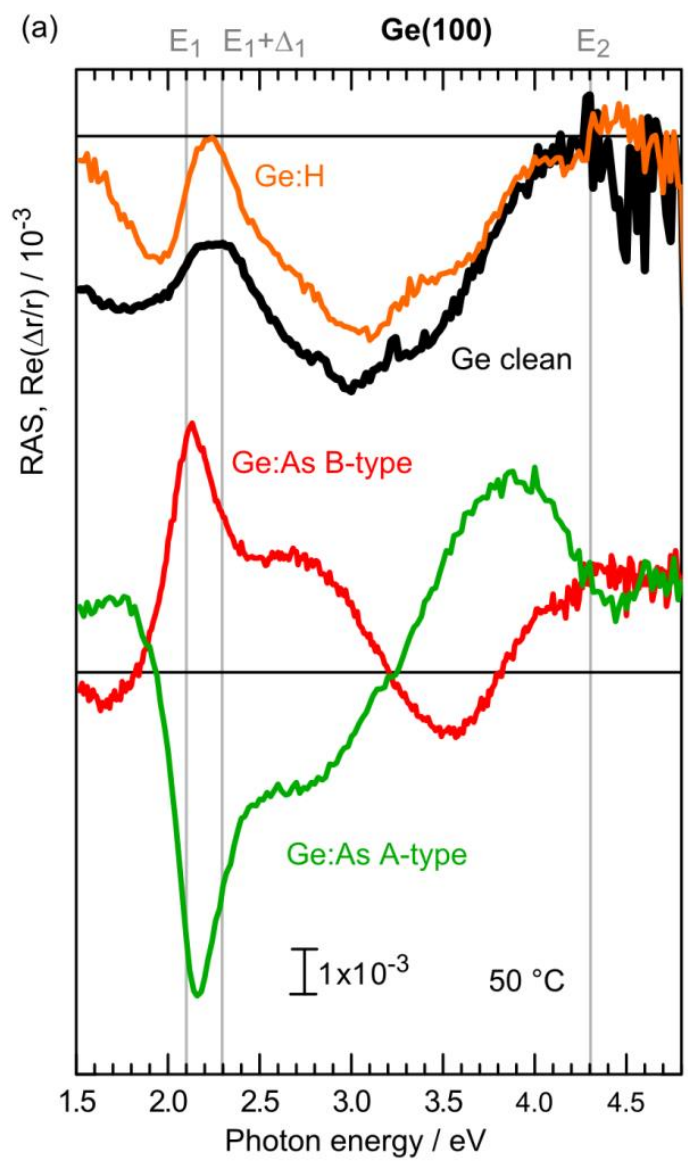

(b)

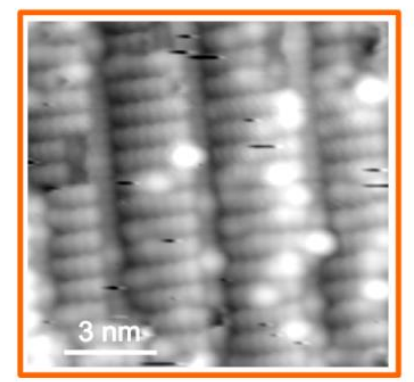

(c)

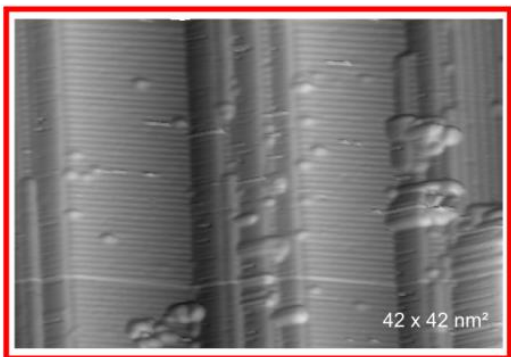

(d)

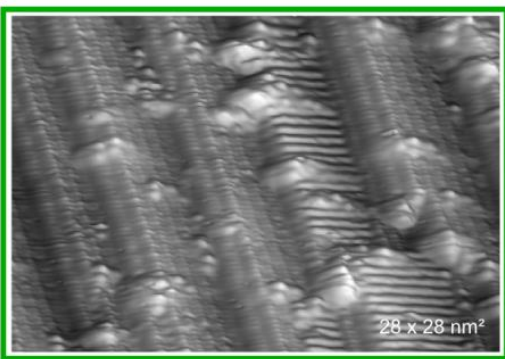

Figure 16. (a) RA spectra of differently terminated vicinal Ge(100) surfaces: monohydride-terminated (orange), clean (black), A-type As-terminated (green) and Btype As-terminated ; vertical lines indicate the interband transitions of $\mathrm{Ge}^{[52]}$; (b-d) benchmarking of the monohydride, A-type As-terminated and B-type As-terminated surfaces, respectively, to STM; data from Ref.s ${ }^{[214,215,230]}$ 


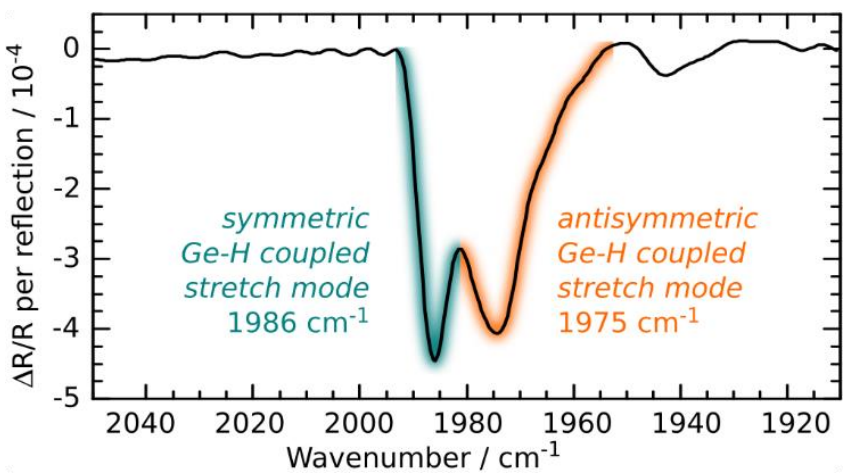

Figure 17. FTIR-ATR spectrum of the monohydride-terminated Ge(100) surface measured in vacuo after annealing in $\mathrm{H}_{2}$ ambient; the absorption band can be assigned to Ge-H coupled stretch modes; data from Ref. ${ }^{[214]}$. 
(a)

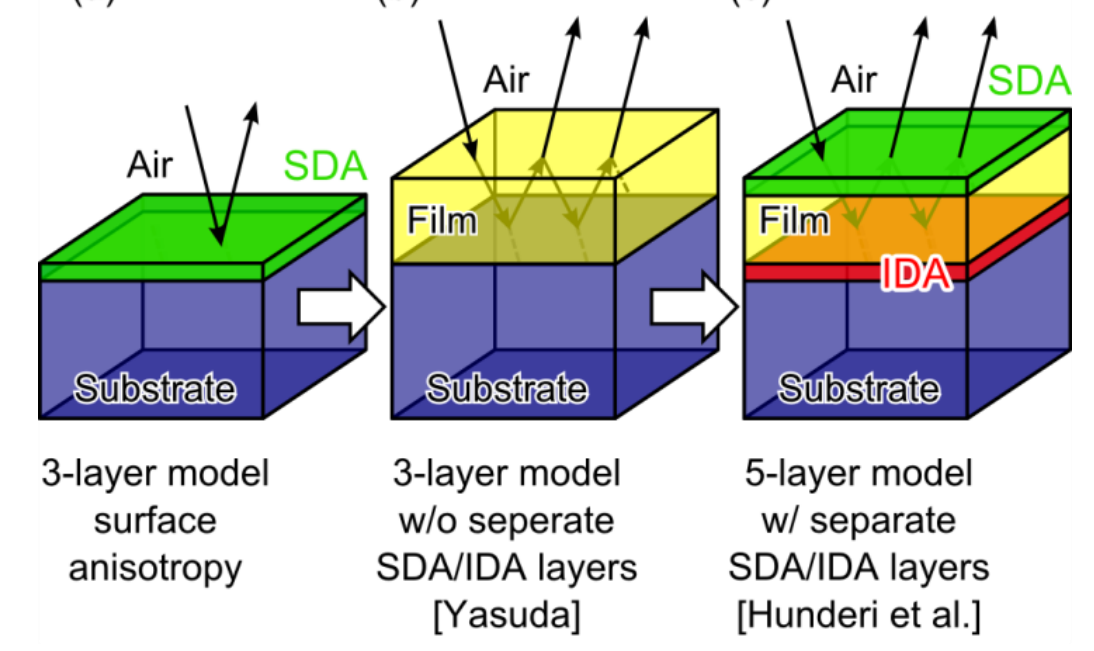

Figure 18. (a) If the RAS signal stems from the surface of a sample, it may be expressed as surface dielectric anisotropy (SDA) assuming a thin optically anisotropic layer on an optically isotropic bulk ${ }^{[442]}$; (b) In order to extract the SDA and the interface dielectric anisotropy (IDA) from RA spectra of heterostructures, Yasuda et al.$^{[239]}$ suggested a 3layer model to account for thickness dependent interference in the film; (c) Hunderi $e t$ $a .^{[242]}$ extended this model to a 5-layer model to account for the interference with additional layers for the SDA and IDA. 


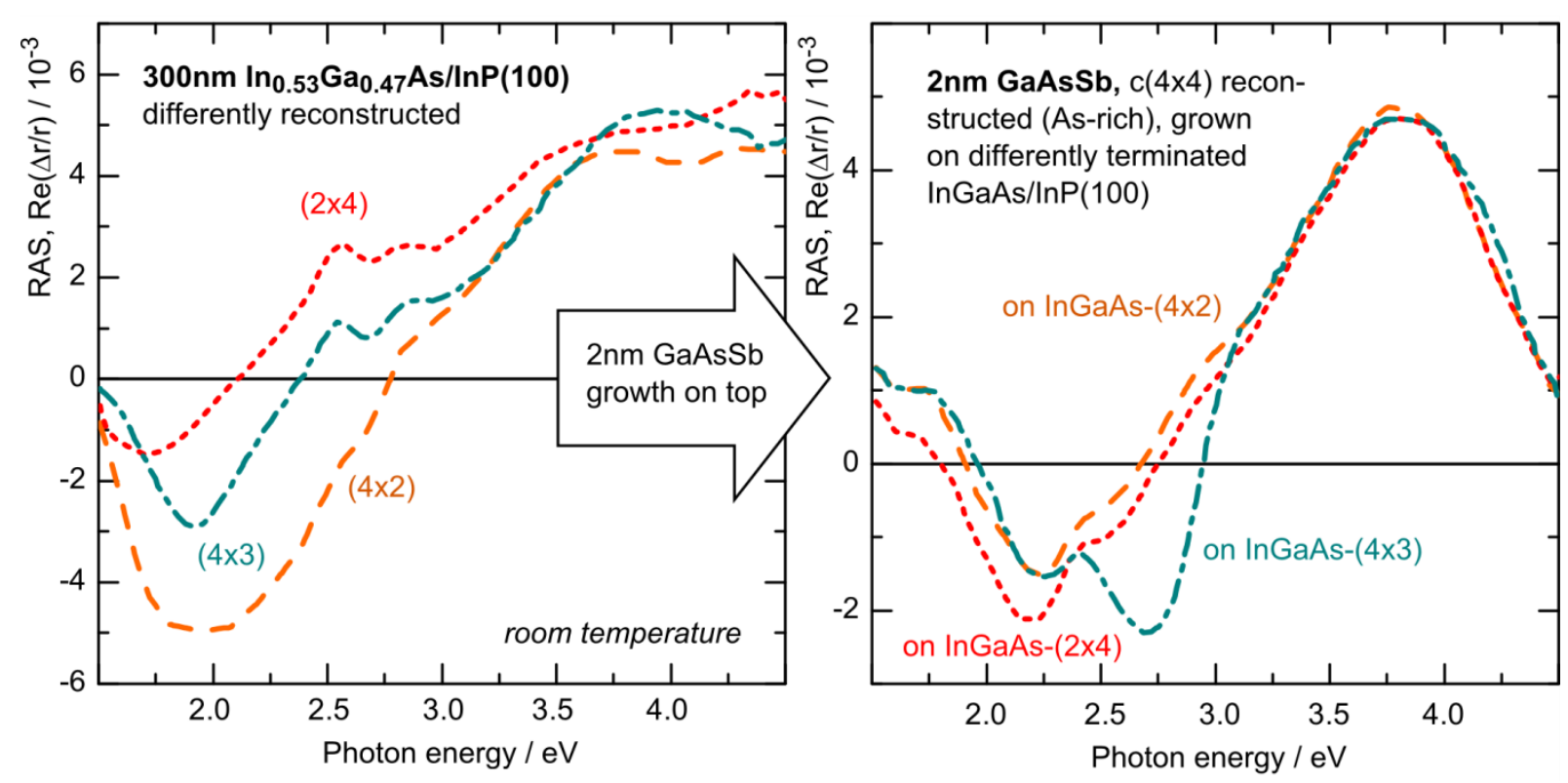

Figure 19. (left) RA spectra of differently reconstructed InGaAs surfaces $(300 \mathrm{~nm}$ grown on InP(100)); (right) RA spectra of $2 \mathrm{~nm}$ GaAsSb grown on top of the differently reconstructed InGaAs/InP(100) samples. All GaAsSb surfaces reconstruct $\mathrm{c}(4 \times 4)$ and are As-rich; data from Ref. ${ }^{[27]}$ 

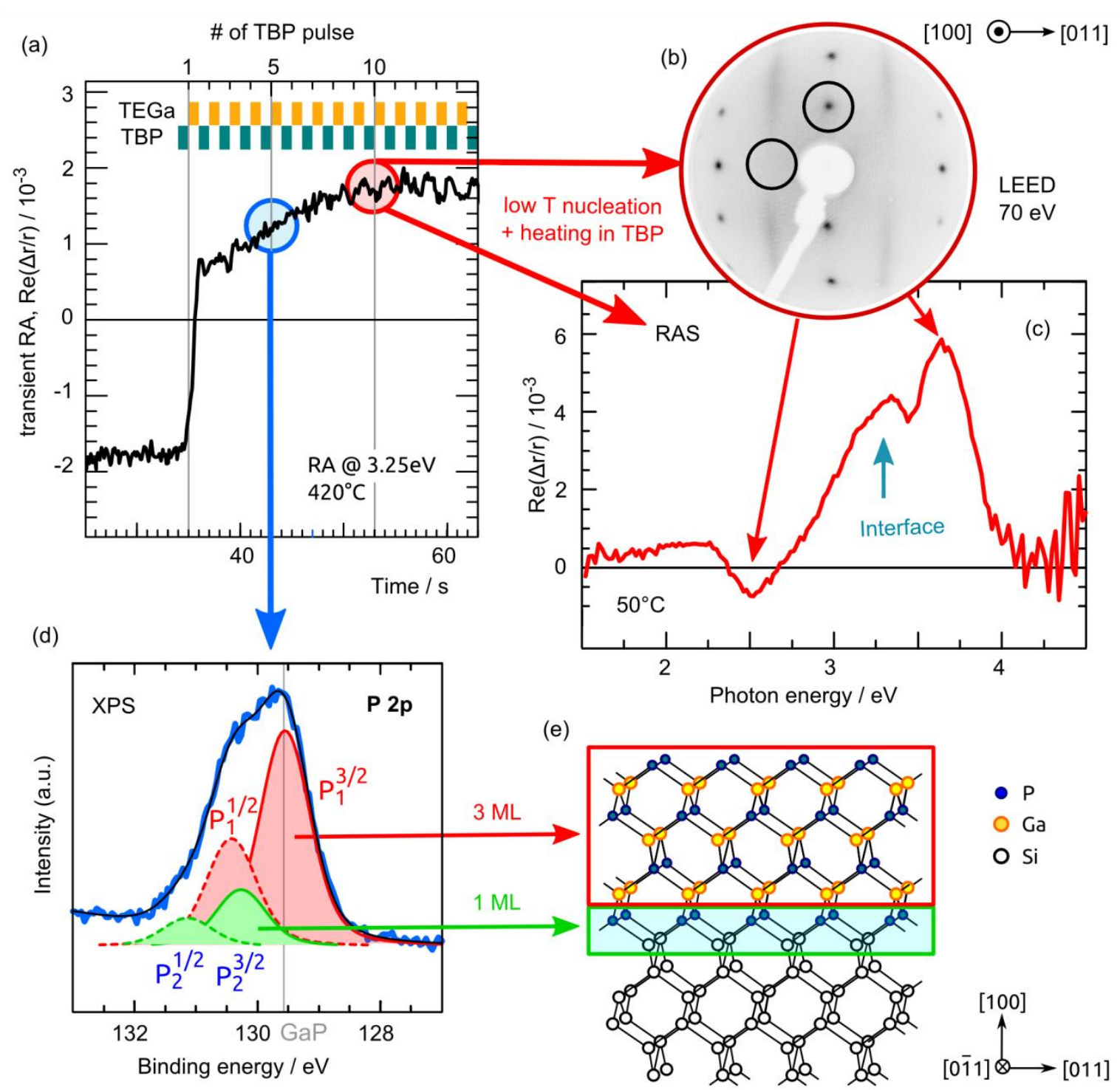

Figure 20. (a) Time-resolved $\mathrm{RA}$ at $3.25 \mathrm{eV}$ measured during pulsed GaP nucleation on majority A-type, monohydride-terminated $\operatorname{Si}(100) 2^{\circ}$ at $420{ }^{\circ} \mathrm{C}$. When stopped after 10 pulse pairs and heated in TBP at $600{ }^{\circ} \mathrm{C}$ (where GaP growth would continue but was omitted here), a single-domain (2x1)-like LEED pattern with streaks along [0-11] occur, which are typical for the P-rich GaP(100) surface. The corresponding RA spectrum (c) already shows the characteristic features of the P-rich $G a P(100)$ surface and an additional contribution, which was assigned to the heterointerface ${ }^{[272]}$; XPS after five pulse pairs reveals a second component in the $P 2 p$ photoemission line (d), which can be attributed to $\mathrm{Si}-\mathrm{P}$ bonds ${ }^{[272]}$; quantification of the XPS data (e) yields about one monolayer (ML) of Si-P bonds and 3 ML GaP; data from Ref. ${ }^{[272]}$ 


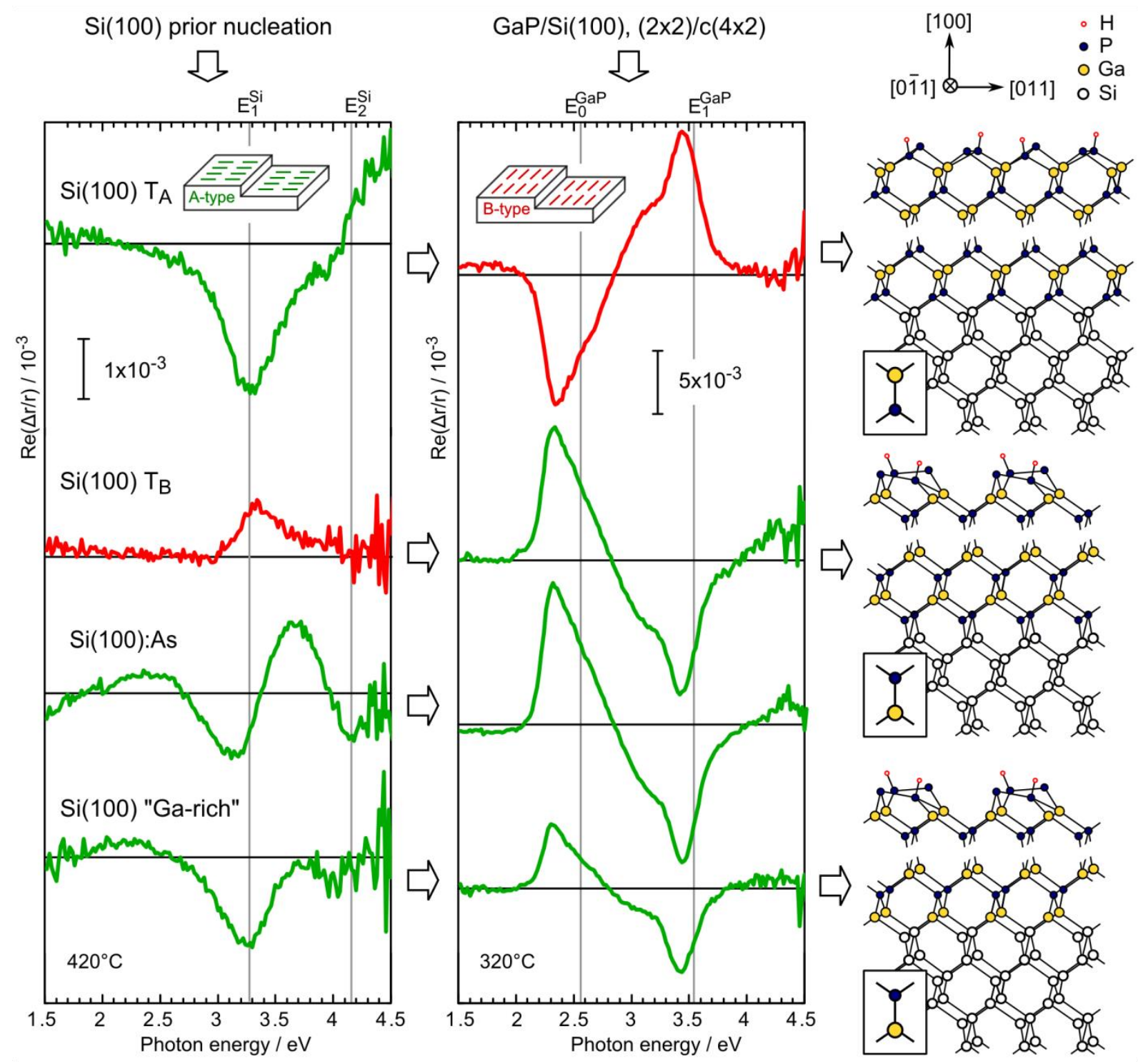

Figure 21. (left column) In situ RA spectra of differently terminated $\mathrm{Si}(100)$ surfaces obtained at $420{ }^{\circ} \mathrm{C}$ directly prior pulsed GaP nucleation: (top) $\operatorname{Si}(100) 2^{\circ}$ with majority A-type domains, (second line) $\mathrm{Si}(100) 2^{\circ}$ with majority B-type domains, (third line) Asmodified $\operatorname{Si}(100) 2^{\circ}$ with majority A-type domains, and (bottom line) $\operatorname{Si}(100) 0.1^{\circ}$ in a "Ga-rich" reactor ${ }^{[260]}$ with A-type domains; (middle column) RA spectra of $35-40 \mathrm{~nm}$ GaP subsequently grown on the corresponding $\mathrm{Si}(100)$ surfaces; (right column) balland-stick models of the heterostructures assuming a simplified abrupt interface model; adapted from Ref. ${ }^{[301]}$, data from Ref. ${ }^{[193,198,208,260]}$; vertical lines indicate interband transitions for $\mathrm{Si}^{[441]}$ and $\mathrm{GaP}^{[440]}$. 


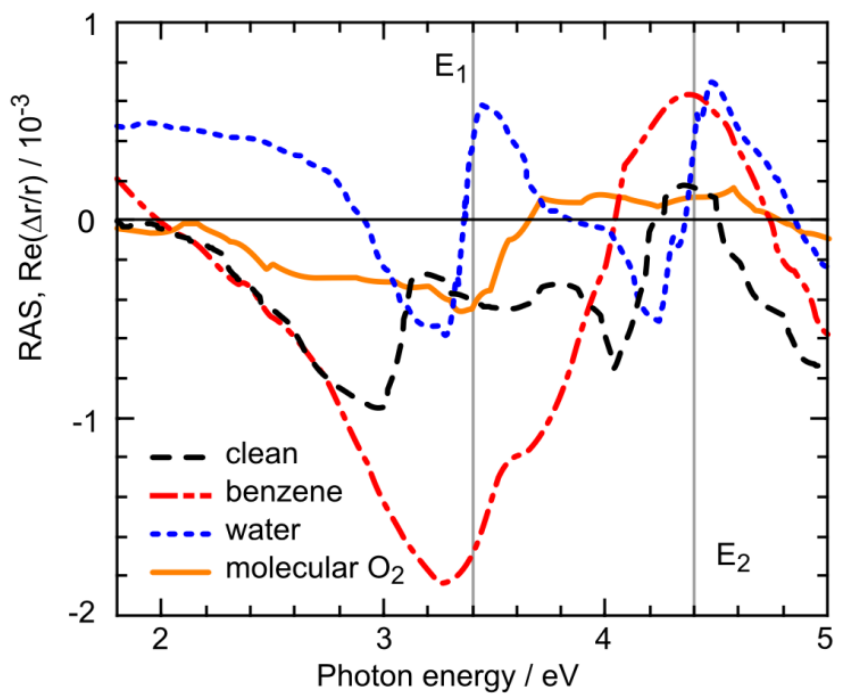

Figure 22. RAS for different adsorbates on the Si(100) surface. Data from Ref. ${ }^{[318]}$. Vertical lines indicate the critical points of $\mathrm{Si}^{[441]}$ 
(a) In-rich $\ln P(100)$ exposed to water

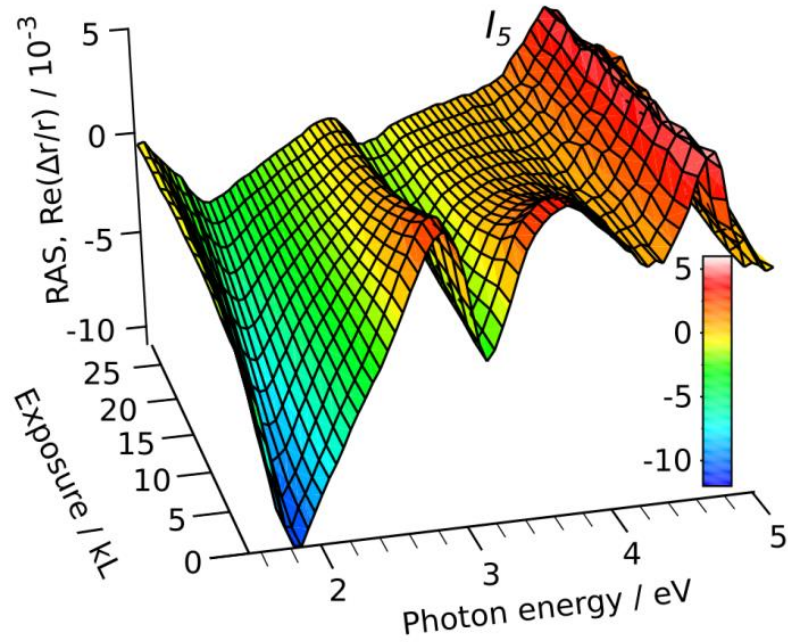

(b) In-rich $\operatorname{In} P(100)$ exposed to oxygen

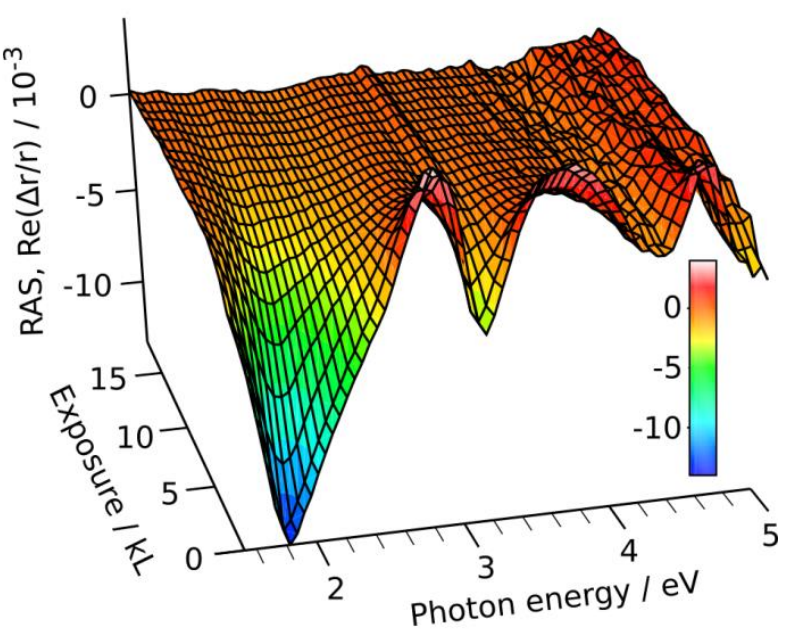

Figure 23. In situ RAS during the exposure of an In-rich surface to water (a) and oxygen (b). data from Ref. ${ }^{[50]} I 5$ denotes a feature related to a surface-modified bulk transition. 


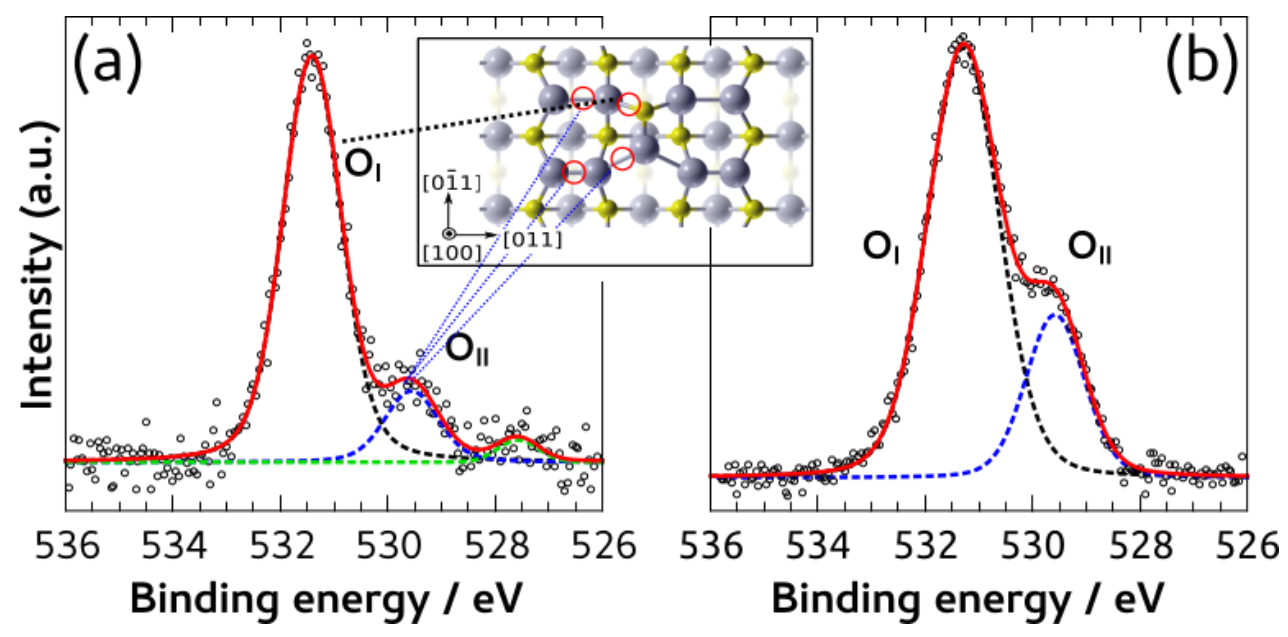

Figure 24. X-ray photoelectron spectroscopy of the $\mathrm{O} 1 \mathrm{~s}$ line after the exposure of the In-rich $\mathrm{InP}(100)$ surface to water (a) and oxygen (b) ; data from Ref. ${ }^{[50]}$ The inset in (a) shows the assignment of spectral features to oxygen binding sites. 
(a) P-rich GaP(100) exposed to water

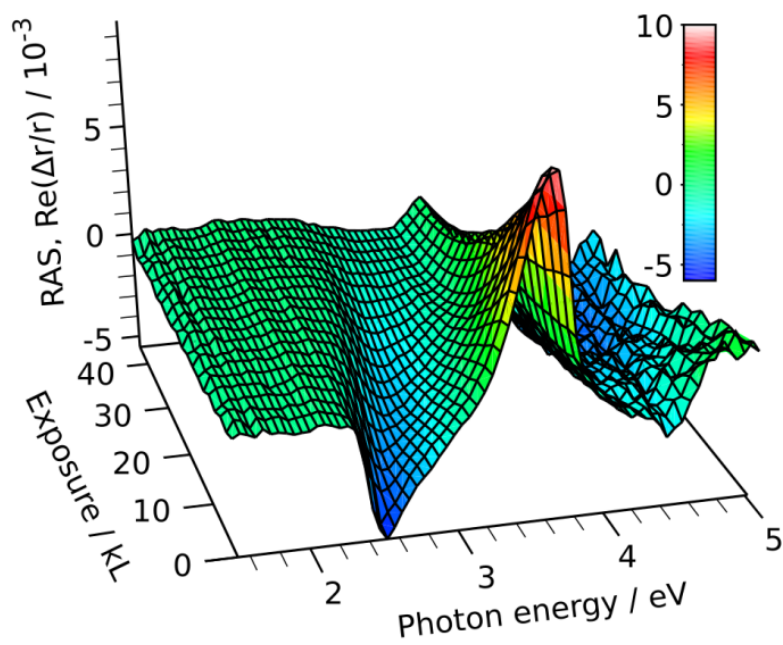

(b) Ga-rich $\mathrm{GaP}(100)$ exposed to water

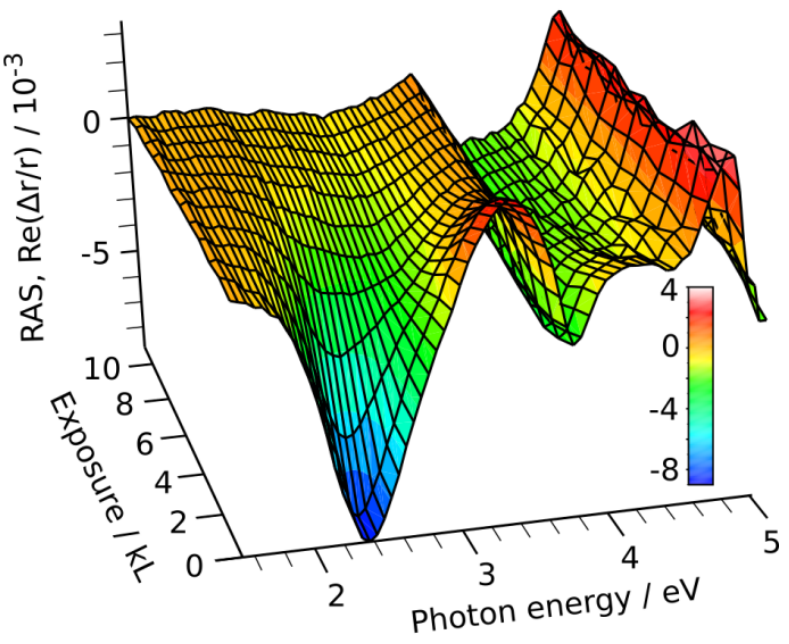

Figure 25. In situ RAS during the exposure of a P-rich (a) and a Ga-rich (b) GaP(100) surface to water. Data from Ref. ${ }^{[326]}$ 

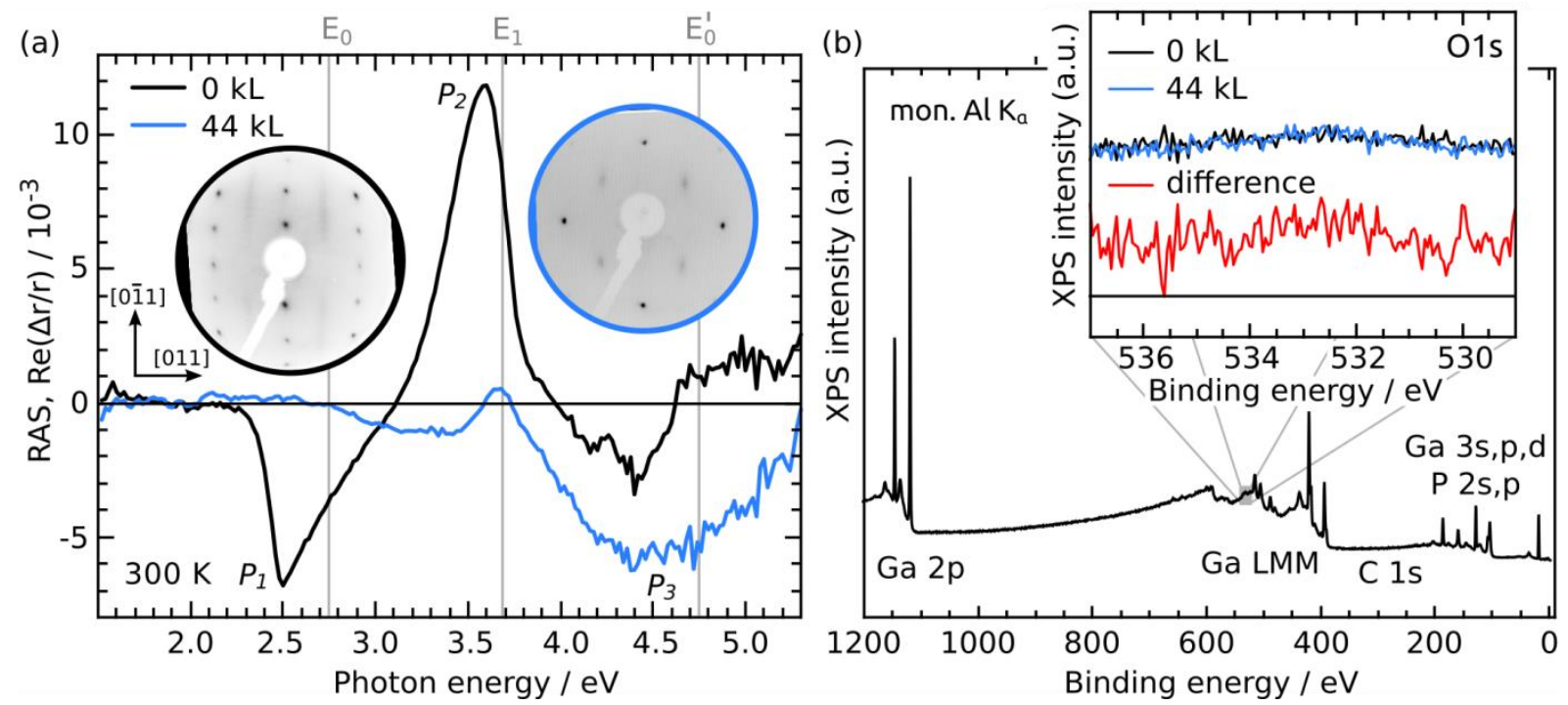

Figure 26. (a) RAS and LEED of P-rich GaP(100) before and after water exposure. The critical point energies are labeled $\mathrm{E}_{\mathrm{i}}$, prominent features of the spectrum $\mathrm{P}_{\mathrm{i}}$. (b) XPS of the Prich surface after water exposure. Data from Ref. ${ }^{[326]}$ 

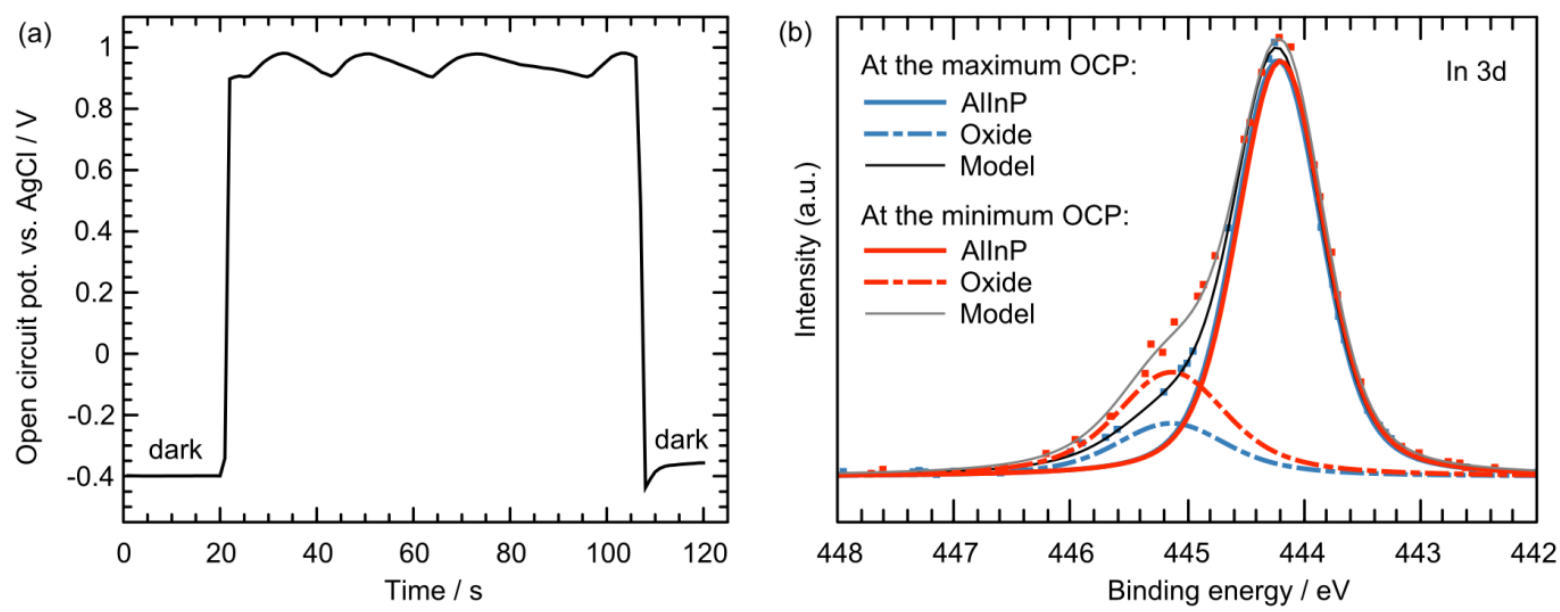

Figure 27. (a) Open-circuit oscillations during functionalization of an AlInP surface for solar water splitting. (b) XPS at different stages of the oscillation ; data from Ref. ${ }^{[14]}$ 


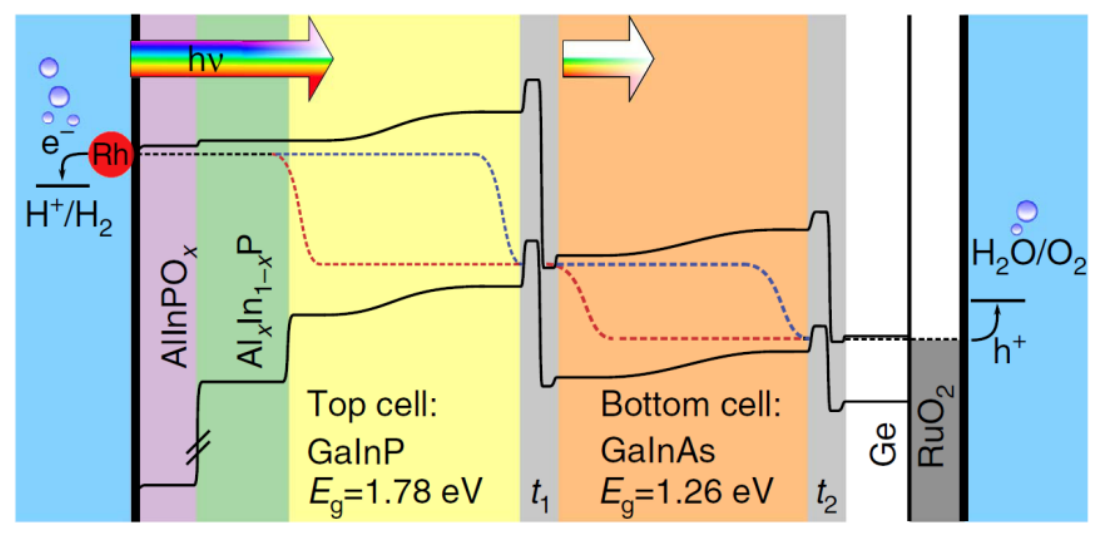

Figure 28. Efficient unassisted water splitting: Energy schematic of the tandem layer structure under illumination yielding a solar-to-hydrogen efficiency of $14 \%$. Solid black lines represent conduction and valence band edges, dashed black lines the Fermi level, dashed blue (red) lines the Quasi-Fermi levels of electrons (holes), and $t_{1}, t_{2}$ the tunnel junctions. Reproduced with permission.. ${ }^{[14]}$ Copyright 2015, CC BY 4.0, M.M. May et al. 


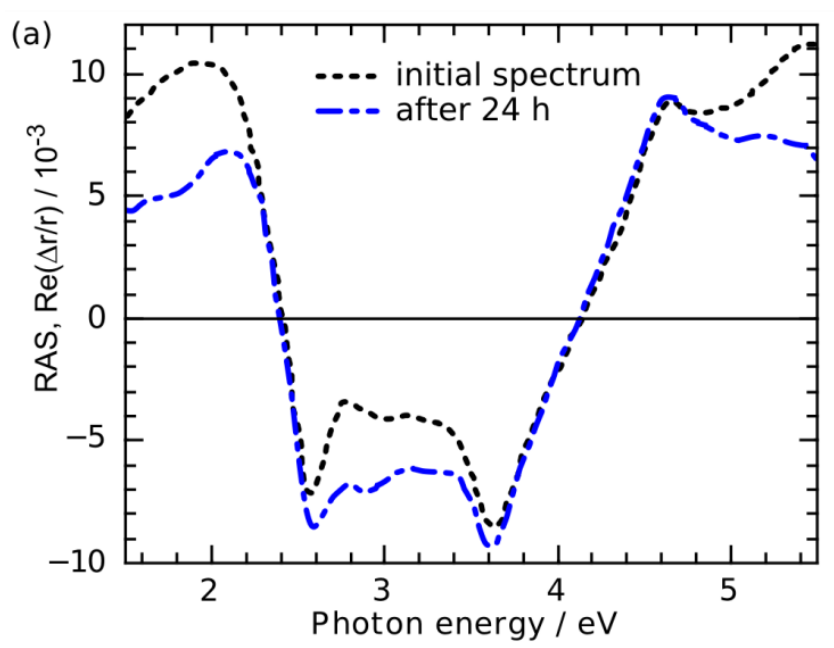

(b)

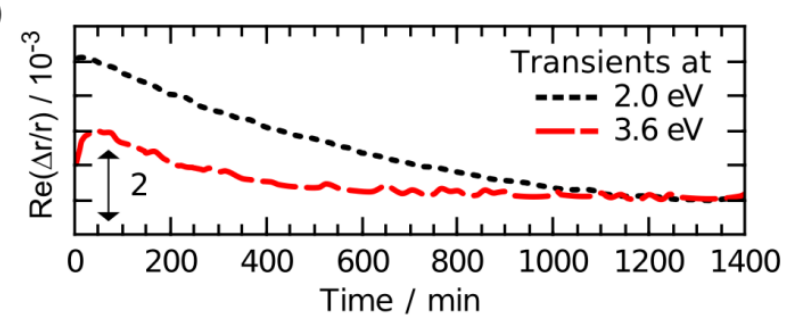

Figure 29. In situ RAS of the $\mathrm{Au}(110)$ surface. (a) Spectra in $\mathrm{H}_{2} \mathrm{SO}_{4}$ directly after immersion and $24 \mathrm{~h}$ later. (b) Time resolved transients at $2 \mathrm{eV}$ and $2.6 \mathrm{eV}$; data from Ref. ${ }^{[345]}$ 

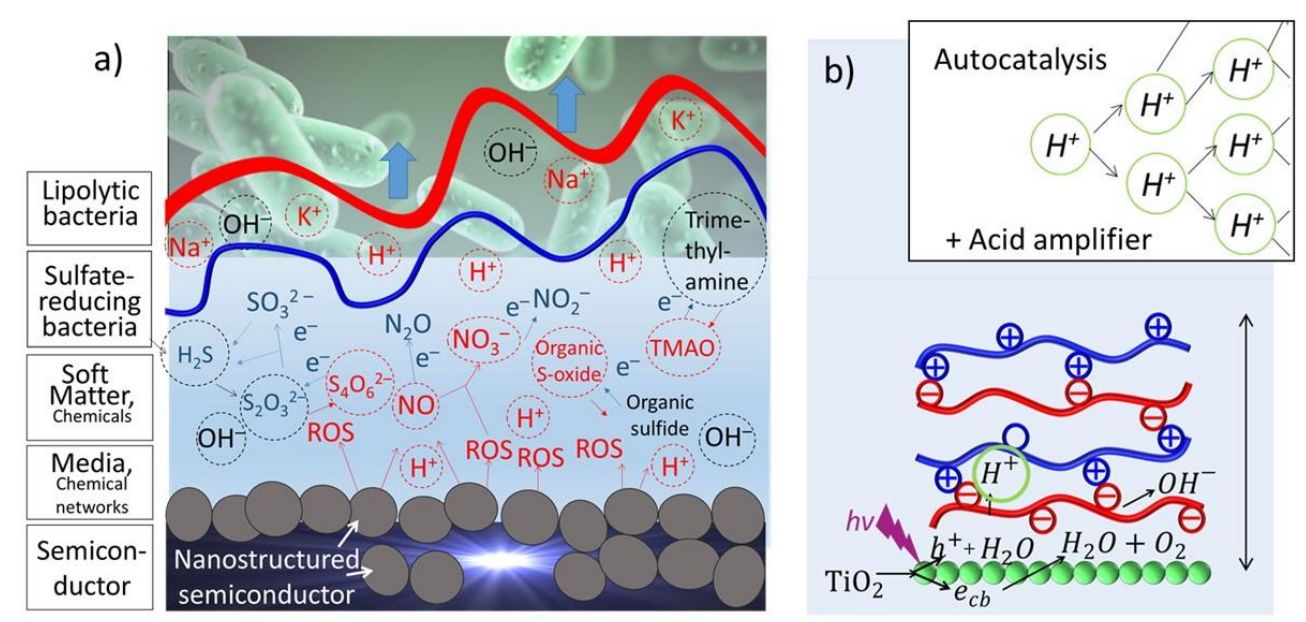

To measure: charge separation ions, $\Delta \mathrm{pH}, \mathrm{ROS}$ activation time relaxation time reversibility ions, $\mathrm{H}^{+}$trapping

Parameters: thickness (h) stiffness roughness hydrophilicity permeability

Bioactivity: biofilm formation

Figure 30. Possible photoreactions at semiconductor / polymer / biological interfaces straightforward for bioinspired nanoscale machinery. (a) Schematic depiction of a photoresponsive system for a nanoscale machinery for biofilm regulation. It consists of semiconductor particles (grey), soft matter and bacteria. Electromagnetic irradiation leads to the production of reactive oxygen species (ROS) and a local change of $\mathrm{pH}$ and other possible reactions depending on the initial composition of the solution and polymers used. The reactions affect the polymer film thickness, roughness, hydrophilicity and local morphology. Possible gradients of species and film properties allow controlling biofilm formation. (b) Surface decoration and photoinitiated light-pH coupled reactions: reactions on $\mathrm{TiO}_{2}$ resulting in a local change of $\mathrm{pH}$. Adapted from Ref. ${ }^{[368]}$. The inset shows that, besides the photogeneration of ions, such as protons, we are focusing on the possibility to amplify ion concentrations, e.g., by adding a chemical amplifier into the system. Abbreviation: TMAO, trimethylamine $\mathrm{N}$-oxide. 

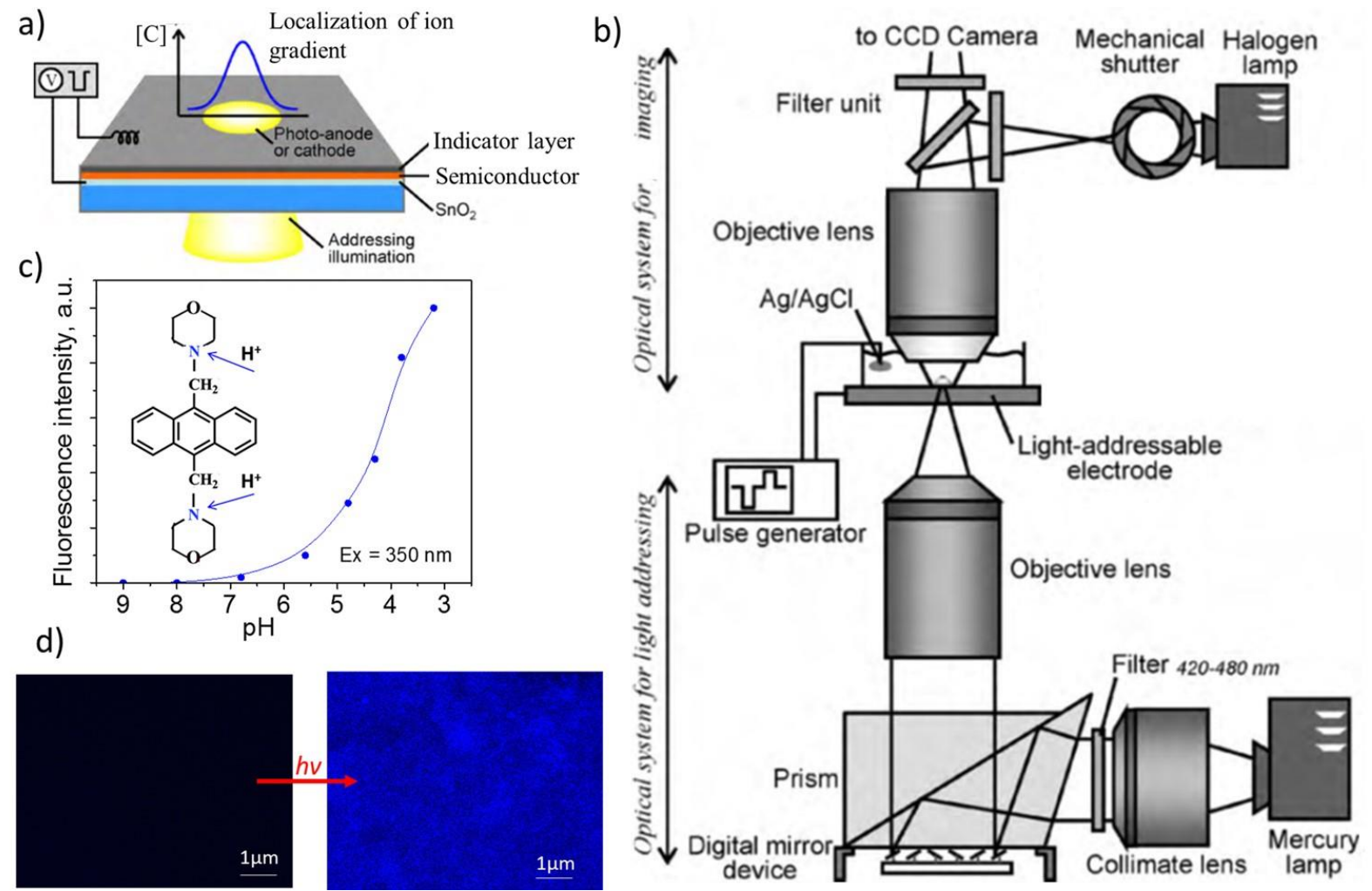

Figure 31. Possible system to detect in situ localized molecules or ion gradients under irradiation using fluorescent assays. (a) Schematic diagram of ion gradient generation with a light-addressable photoelectrode. (b) Experimental setup for in situ light-addressing and ion imaging. Two separate optical systems can be used; a standard fluorescence upright microscope and a reduced projection exposure system equipped under the microscope stage. A patterned illumination reflected from a digital micromirror device may be projected onto the electrode substrate. Fluorescence emission of the ion, e.g., $\mathrm{pH}$ indicator, is monitored by a cooled CCD camera attached to the microscope. The measured fluorescence intensities may be converted into ion concentration or $\mathrm{pH}$ changes using a calibration curve obtained in advance (adapted from Ref. ${ }^{[406]}$ ). (c) Calibration of the fluorescent $\mathrm{pH}$ indicator, and initial chemical formula to point to the protonation sites. (d) Confocal fluorescence images of the surface of $\mathrm{TiO}_{2}$, with the fluorescent $\mathrm{pH}$ dye (left) before and (right) after $10 \mathrm{~s}$ of irradiation. Adapted from Ref. ${ }^{[370]}$ 
a)

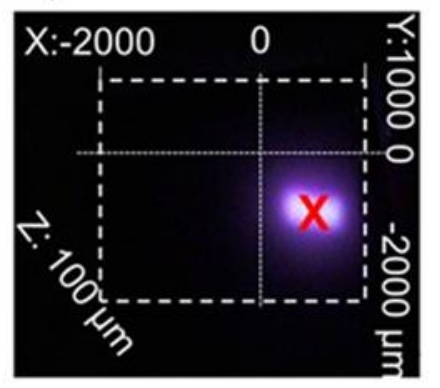

b)

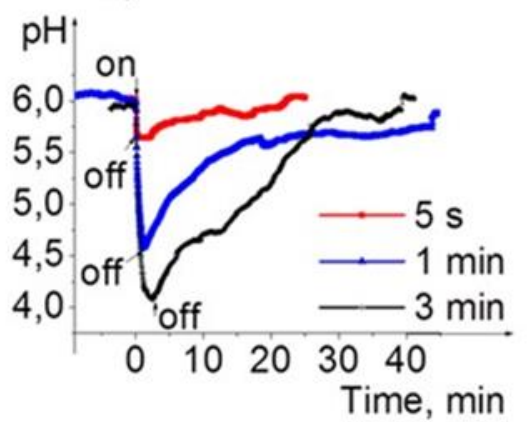

c)

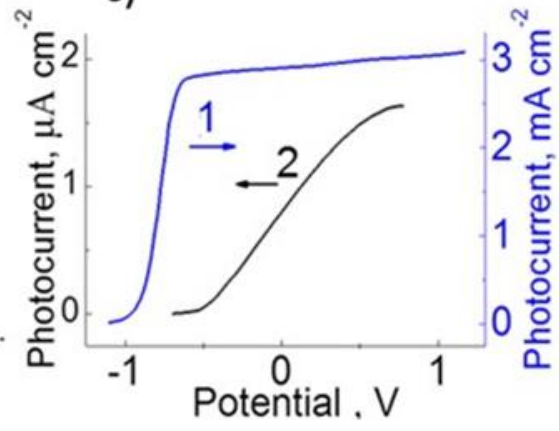

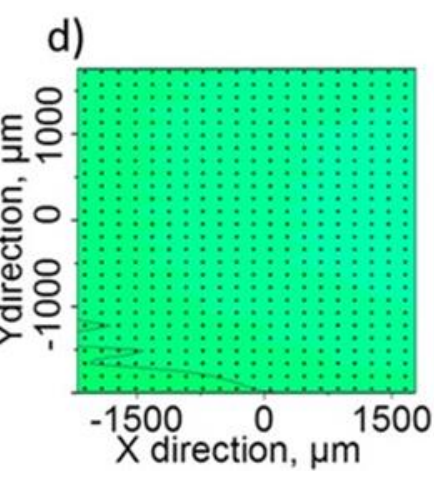

e)

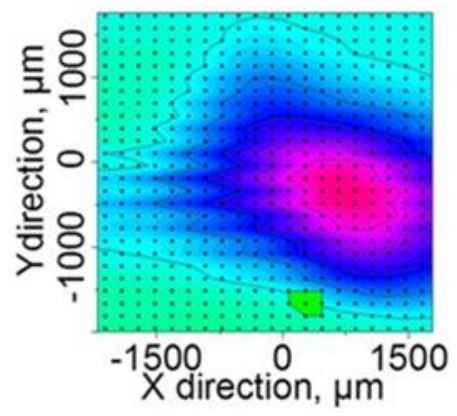

Figure 32. In situ local $\mathrm{pH}$ activity over a $\mathrm{TiO}_{2}$ surface. (a) Optical image of the surface during irradiation and measurements by the scanning ion selective electrode technique (SIET) for mapping of the activity of $\mathrm{H}^{+}$ions over the $\mathrm{TiO}_{2}$ film under local irradiation. (b) Temporal evolution of the $\mathrm{pH}$ over the mesoporous $\mathrm{TiO}_{2}$ film in the center of the irradiation zone $(\mathrm{X}$ in (a); on-illumination is switched on, off-illumination is switched off). (c) Photocurrent of low and high photoactivity $\mathrm{TiO}_{2}$ indicating that for SIET study of the high photoactivity surface is more suited to detect the effect, but for soft matter actuation low photoactivity $\mathrm{TiO}_{2}$ is preferable, not to degrade the polymer assembly. (d-f) SIET maps of proton activity (d) before and (e) during the exposure of certain localized areas. Adapted from Ref. ${ }^{[368]}$ 

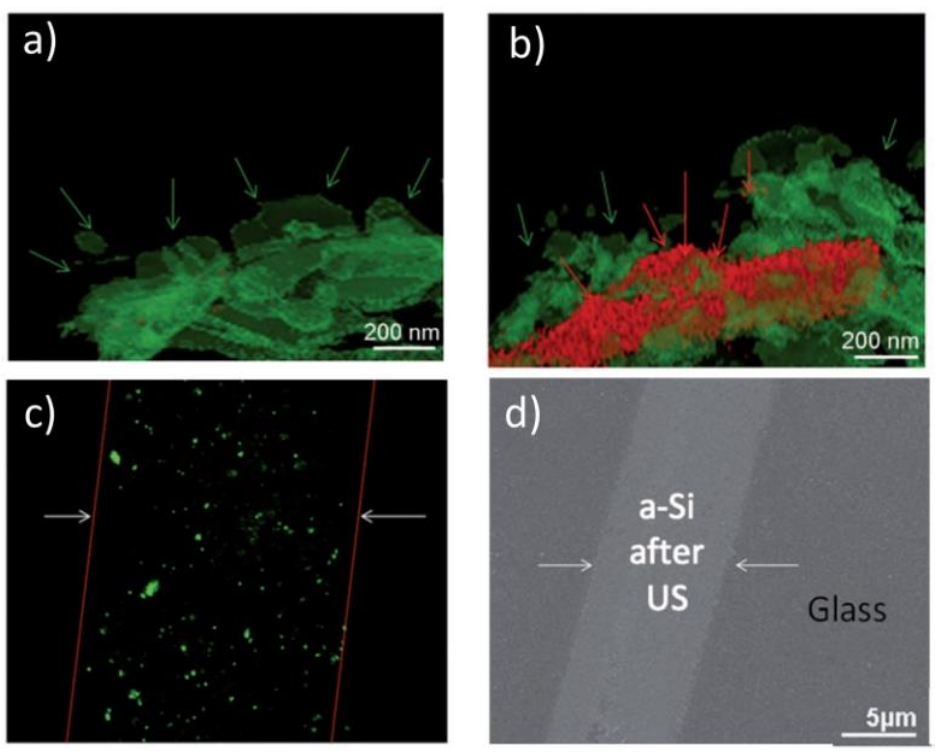

Figure 33. (a-c) In situ $\mu$-confocal photoluminescence( $\mu$-CPL) spectra (fluorescence mode) of silicon after $30 \mathrm{~min}$ of sonochemical modification $\left(20 \mathrm{kHz}, 57 \mathrm{Wcm}^{-2}\right)(\mathrm{a}-\mathrm{b})$ of crystalline silicon wafer (a) immediately after modification, and (b) followed by ageing; (c) amorphous silicon (a-Si) deposited on glass; (d) corresponding SEM picture; green and red arrows show side views of the PL spectra. Adapted from Ref. ${ }^{[409]}$ 

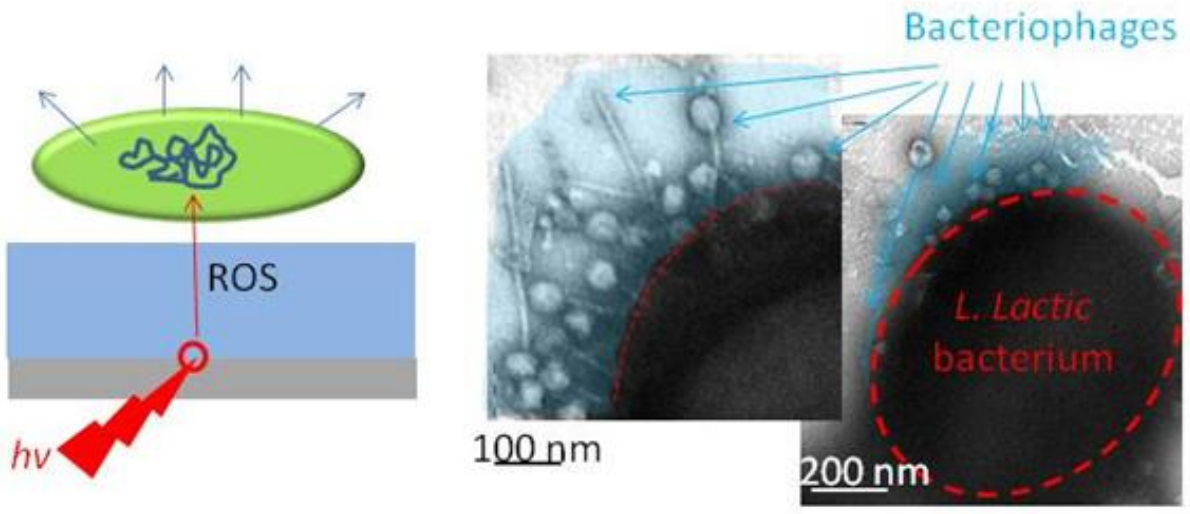

Figure 34. Schematic and transmission electron microscopy (TEM) image demonstrating the effect of ROS generated at a semiconductor on the metabolism of lysogenic L. lactic bacteria (i.e., containing a bacteriophage gene in their deoxyribonucleic acid (DNAs). The opening of the cell envelope and the release of viruses is observed. Adapted from Ref. ${ }^{[16]}$. 


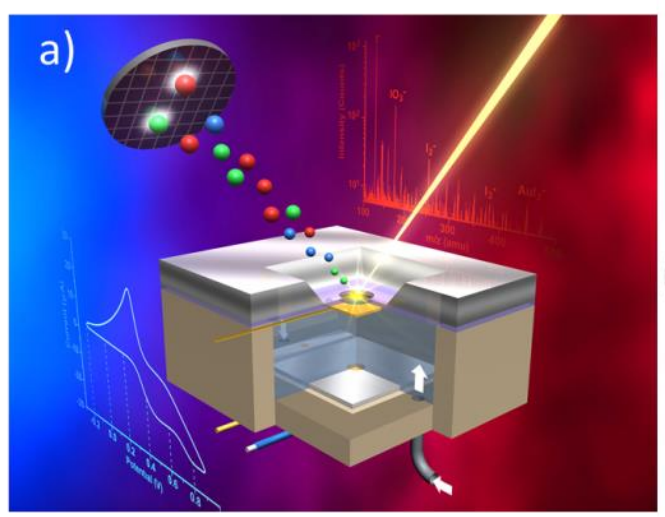

c)

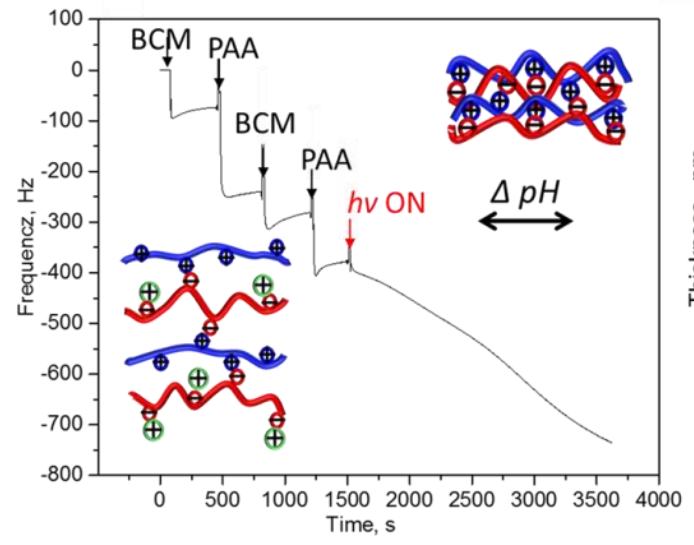

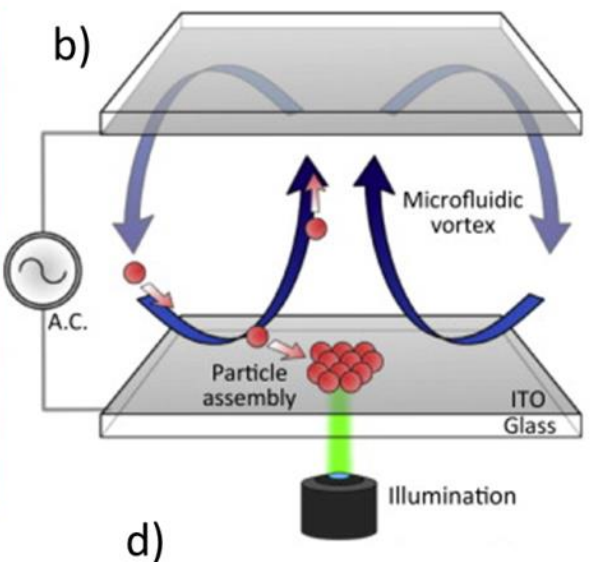

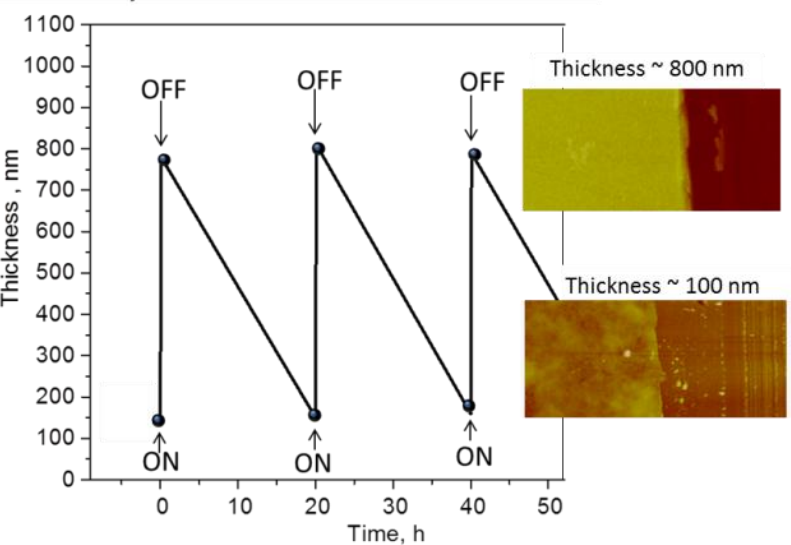

Figure 35. (a) A microfluidic probe allows real-time imaging of the electrode-liquid electrolyte interface. Adapted from Ref. ${ }^{[422]}$. (b) Prospective system to guide particle movement and real-time microfluidic vortex detection, i.e., by gas formation and effecting particle migration on a surface. Adapted from Ref. ${ }^{[443]}$. (c) In situ quartz crystal microbalance (QCM) study of LbL PEs assembly and their activation under irradiation resulting in water attraction into the $\mathrm{LbL}$ and (d) LbL thickness change before and after irradiation. Abbreviation: BCM, block copolymer micelles; PAA, poly(acrylic acid) (PAA). Adapted from Ref. ${ }^{[368,370]}$ 

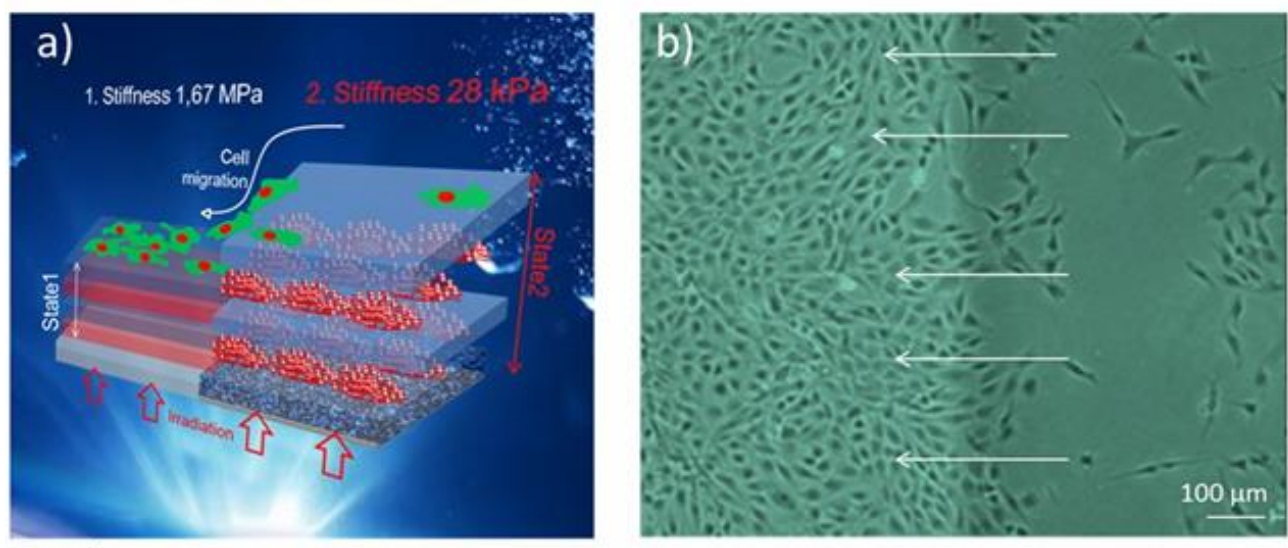

c)
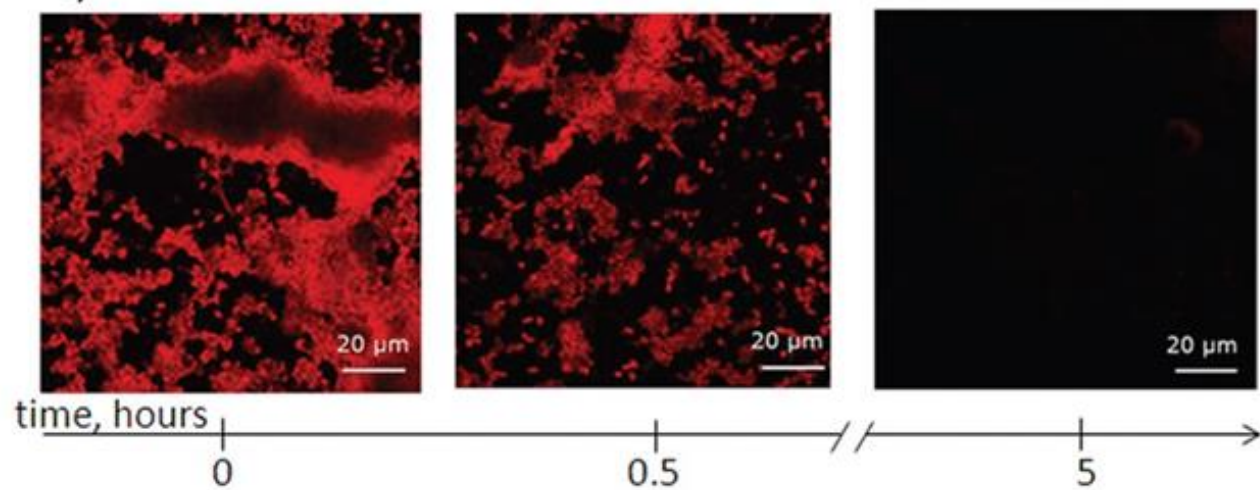

Figure 36. In situ bioresponse on soft matter actuation. (a, b) Examples of consequences of a dynamic concept of modulation of a multilayer coating on titania using light-pH coupled stimuli to regulate cells on the surface: during irradiation the stiffness changes drastically and cells start to migrate across the surface. Adapted with permission from Ref. ${ }^{[370]}$ (c) Actuating coating can be used as an antifouling approach preventing bacteria from growing on the surface. An example of a self-regulating system is presented: Lactic bacteria are grown on the surface and change the $\mathrm{pH}$. The coating then changes its thickness fast and "pushes off" bacteria from the surface. Adapted with permission from Ref. ${ }^{[382]}$ 

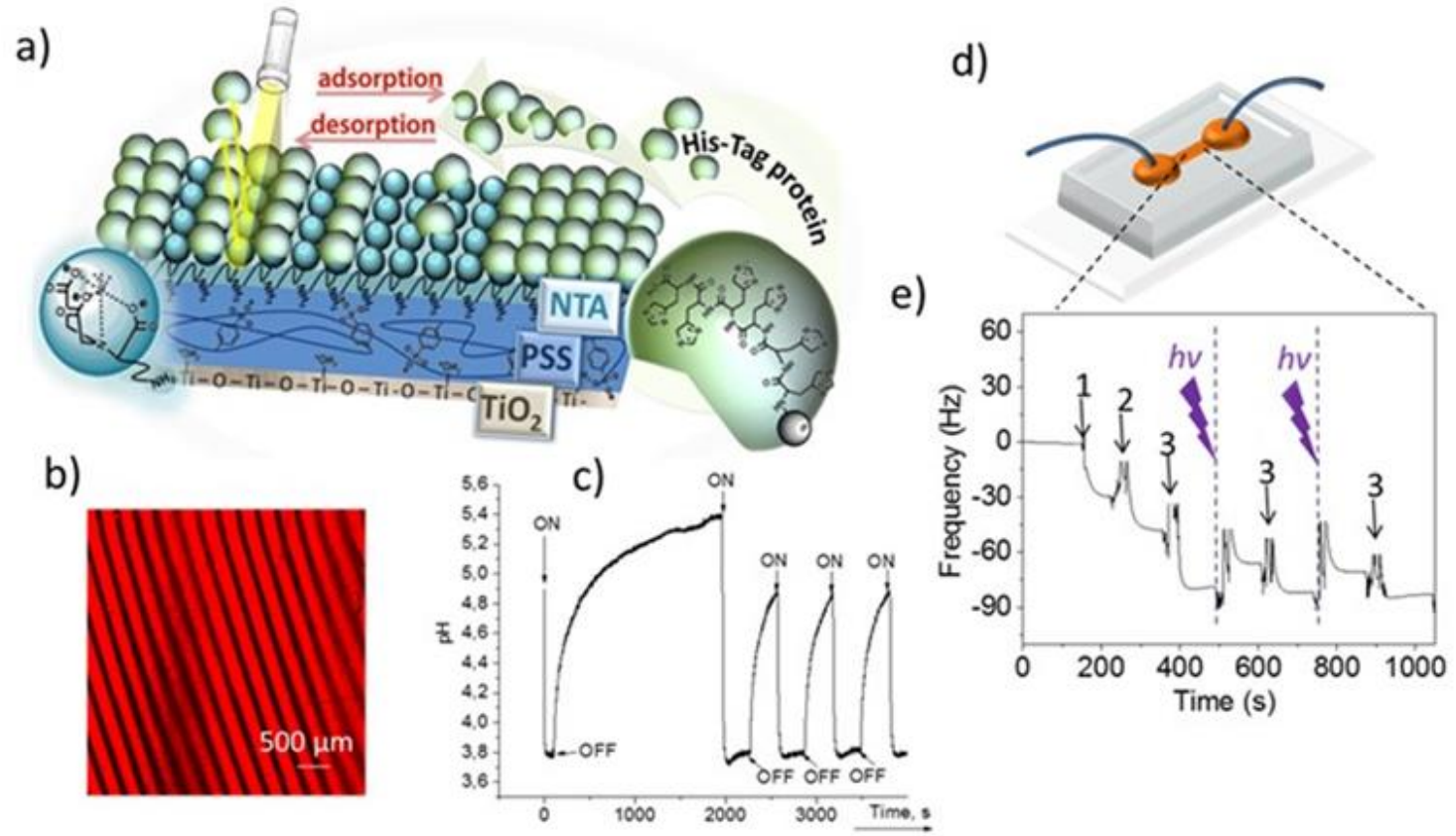

Figure 37. (a) Schematic illustration of the release of proteins from the surface using light-pH coupled stimuli on titania: LbL assembly of strong polyelectrolyte layers, polystyrene sulfonate (PSS), and $\mathrm{pH}$-responsive and nickel-nitrilotriacetic acid $\left(\mathrm{Ni}^{2+}-\mathrm{NTA}\right)$ layers for (poly)histidine-tagged (His-Tag) protein recognition and manipulation. (b) System $\mathrm{TiO}_{2} /(\mathrm{PSS} / \mathrm{NTA} / \mathrm{His}-\mathrm{Tag})_{4} / \mathrm{PSS}$ after irradiation through a photomask allows local delivery / desorption of proteins. (c) Reversible and multicycle activity is detected from real time evolution of the activity of $\mathrm{H}^{+}$-ions over the $\mathrm{TiO}_{2} / \mathrm{PSS} / \mathrm{NTA}$ surface. "ON" is a time point of irradiation switched on and "OFF" - switched off. (d) Microfluidic chamber to study His-Tag protein light induced modulation / desorption on the $\mathrm{TiO}_{2} / \mathrm{LbL}$ surface. (e) In situ QCM frequency change during the adsorption of (1) PSS, (2) NTA, (3) His-Tag protein; $h v$ corresponds to the moment of short term (1s) irradiation and results in protein desorption. Cycles of adsorption, light induced desorption are repeated several times. Cycles of adsorption, light induced desorption are repeated several times. Adapted with permission from Ref. $^{[369]}$ 

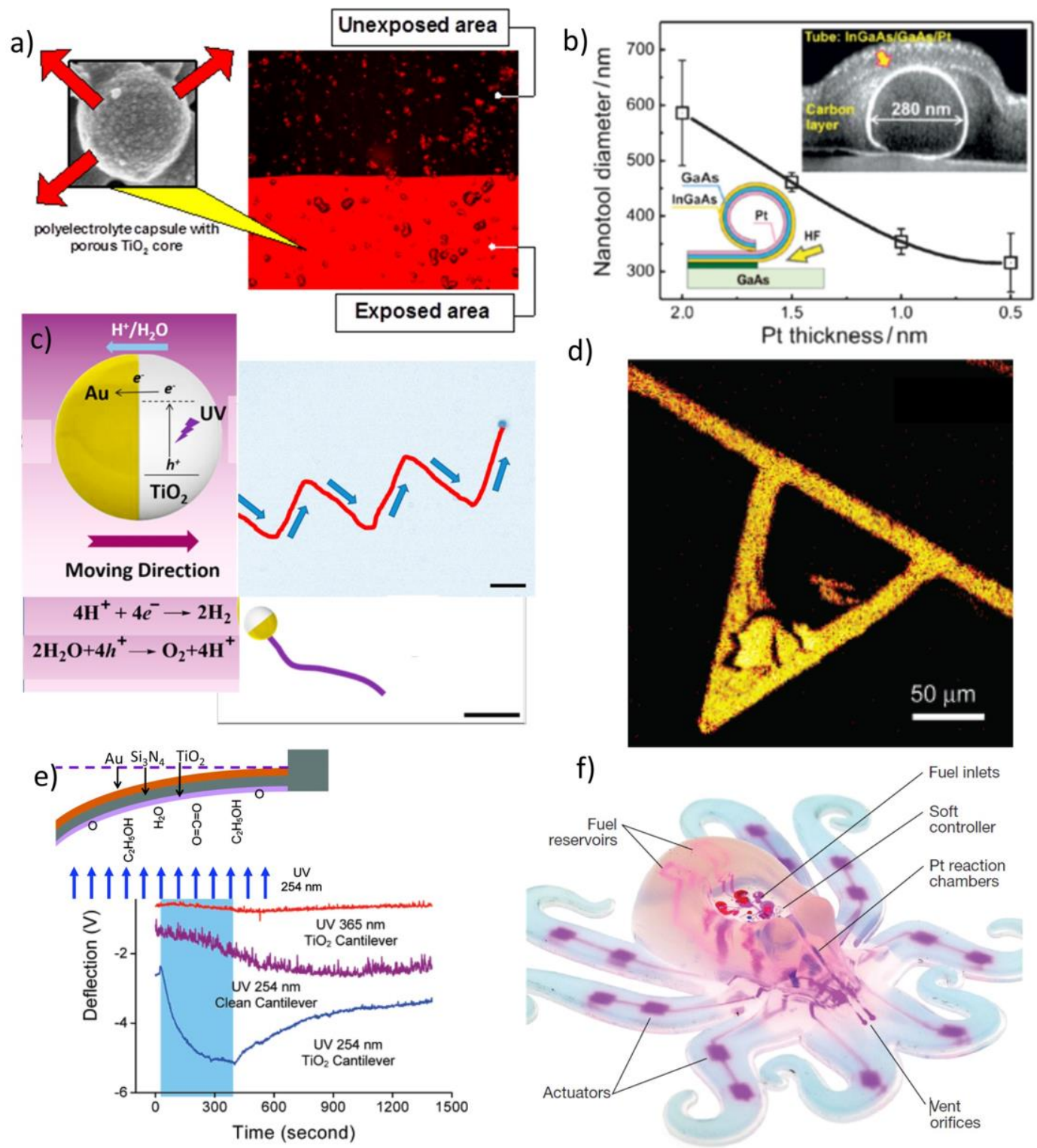

d)
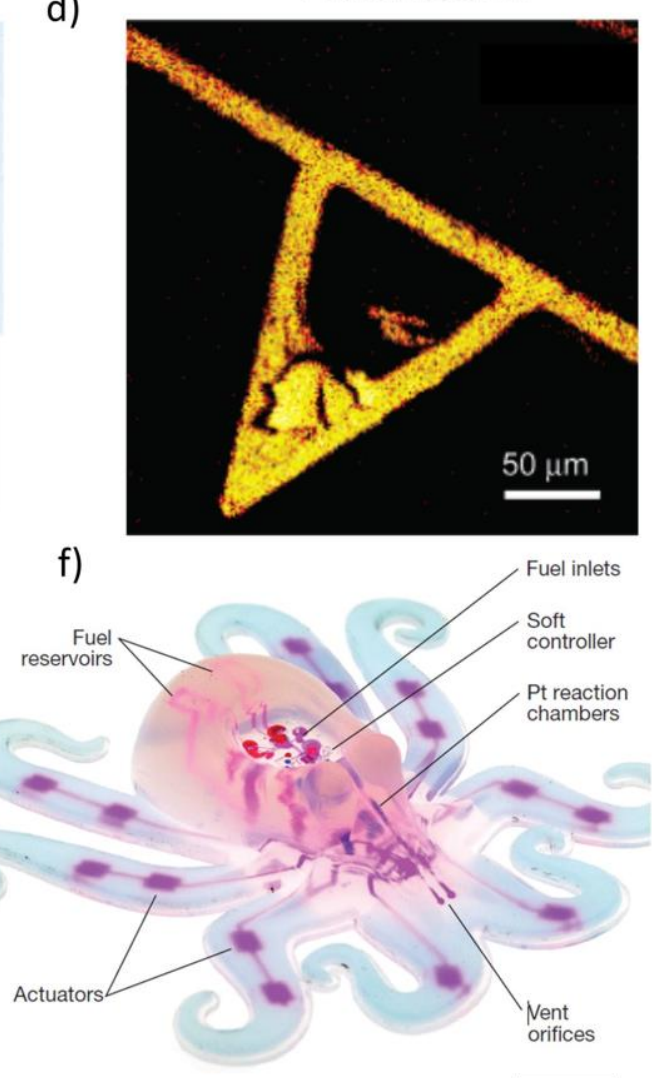

Figure 38. (a) Titania-based capsules and LbL organized polyelectrolyte shell become permeable under irradiation and can locally release active components from the surface, which could regulate cell activity on the surface. Adapted with permission from Ref. ${ }^{[421]}$ (b) Scalability of the diameter of rolled-up nanotubes consisting of hybrid heteroepitaxial catalytic InGaAs $(3 \mathrm{~nm}) / \mathrm{GaAs}(3 \mathrm{~nm}) / \mathrm{Pt}$ thin films. Bottom inset shows the rolled-up fabrication process by selective under-etching of the sacrificial AlAs $(20 \mathrm{~nm})$ layer. Top inset depicts a SEM image containing a focused ion beam cut of an individual tube composed of InGaAs/GaAs/Pt $(0.5 \mathrm{~nm})$. Adapted from Ref. ${ }^{[444]}$ (c) Schematic of catalytic $\mathrm{TiO}_{2}$-Au Janus micromotors powered by UV light in water and time-lapse images showing the stimuli guided propulsion of an $\mathrm{Au}-\mathrm{Ni}-\mathrm{TiO}_{2}$ micromotor under $40 \times 10^{-3} \mathrm{~W} / \mathrm{cm}^{2} \mathrm{UV}$ light. Scale bar, $10 \mu \mathrm{m}$. Adapted from Ref. ${ }^{[429]}$ (d) The elemental distribution images of a $\mathrm{TiO}_{2}$ modified microcantilever collected using secondary ion signals of $\mathrm{Ti}^{+}$; and (e) bending of a $\mathrm{TiO}_{2}$ modified microcantilever exposed to UV radiations at 365 and $254 \mathrm{~nm}$, and the bending of an unmodified microcantilever exposed to UV radiation at $254 \mathrm{~nm}$ (inset shows scheme for ethanol-fueled photocatalytic microengines based on $\mathrm{TiO}_{2}$ modified AFM microcantilevers). Adapted from Ref. ${ }^{[428]}$ (f) Fuel reaction is very prospective in semiconductor surface for fully 
soft, autonomous robot assembly, shown as example in image. Scale bar, $2 \mathrm{~mm}$. Adapted from Ref. ${ }^{[431]}$ 
Table1. Specific examples of detection of reactive species on semiconductors

\begin{tabular}{|c|c|c|c|c|c|}
\hline Analyzed material & $\begin{array}{l}\text { Detected } \\
\text { species }\end{array}$ & Methods for detection & $\begin{array}{l}\text { Light source for sample } \\
\text { irradiation }\end{array}$ & $\begin{array}{l}\text { Reagents and additional features in methodology of photogenerated species } \\
\text { detection }\end{array}$ & Reference \\
\hline $\begin{array}{l}\left.{ }^{[445,}, 446\right] \\
\mathrm{CeO}_{2},{ }^{[446]} \mathrm{Ag}^{[447]}\end{array}$ & $\mathrm{OH} \cdot, \mathrm{O}_{2^{-}},{ }^{1} \mathrm{O}_{2}$ & $\begin{array}{l}\text { Colorimetric probe method } \\
\text { (UV-vis }{ }^{\text {a) }} \text { spectroscopy) }\end{array}$ & $\begin{array}{l}18 \text { W black-light blue lamps } \\
4 \text { W compact UV lamp }(365 \\
n m)^{[446,447]}\end{array}$ & $\begin{array}{l}=\mathrm{OH} \cdot \text { indicator- } \mathrm{p} \text {-nitrosodimethylaniline }(\lambda=440 \mathrm{~nm}))^{[445]} \mathrm{p} \text {-chlorobenzoic acid }{ }^{[446,447]} \\
\mathrm{O}_{2}{ }^{-} \text {indicator }-2,3-\text { bis }(2-\text {-methoxy-4-nitro-5-sulfophehyl)-2H-tetrazolium-5- } \\
\text { carboxanilide }(\lambda=470 \mathrm{~nm}) ;{ }^{[445,446,447]}{ }^{1} \mathrm{O}_{2} \text { indicator - furfuryl alcohol }{ }^{[446,447]}\end{array}$ & {$[445,446,447]$} \\
\hline $\mathrm{TiO}_{2}, \mathrm{ZnO}$ & $\mathrm{H}_{2} \mathrm{O}_{2}$ & $\begin{array}{l}\text { Polarographic measurements } \\
\text { Fluorescence measurements } \\
\text { lodide method }\end{array}$ & $450 \mathrm{~W}$ Xe lamp & $\begin{array}{l}\text { 1) } \mathrm{TiO}_{2} \text { suspension }(0,5 \mathrm{~g} / \mathrm{L}) \text { with } \lambda=350 \mathrm{~nm} \text { and } \mathrm{ZnO} \text { suspension (1mM; } \mathrm{pH} 7.7 ; \mathrm{O}_{2} \\
\text { bubbling) with } \lambda=320-330 \mathrm{~nm} \\
\text { 2) P-hydroxyphenylacetic acid, horseradish peroxidase and detection of } \\
\text { dimerization of p-phenylacetic acid at } \lambda_{\mathrm{ex}}=315 \mathrm{~nm}, \lambda_{\mathrm{em}}=406 \mathrm{~nm} \\
\text { 3) lodide reagent }\left(0,4 \mathrm{M} \mathrm{KI}, 0,06 \mathrm{M} \mathrm{NaOH}, \sim 10^{-4} \mathrm{M}\left(\mathrm{NH}_{4}\right)_{2} \mathrm{MoO}_{4}\right)\end{array}$ & [448] \\
\hline $\begin{array}{l}\mathrm{TiO}_{2},{ }^{[499]} \\
\mathrm{TiO}_{2}: \mathrm{Sm}^{3+[450]}\end{array}$ & $\mathrm{OH} \bullet$ & Fluorescence measurements & $\begin{array}{l}200 \text { W high-pressure mercury } \\
\text { lamp } \\
160 \text { W high-pressure } \\
\text { mercury lamp }{ }^{[450]}\end{array}$ & 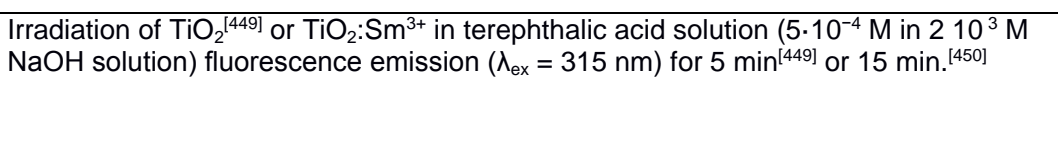 & {$[449,450]$} \\
\hline $\mathrm{Cu}_{2} \mathrm{O} / \mathrm{SnO}_{2}$ & $\mathrm{H}_{2} \mathrm{O}_{2}$ & $\mathrm{DPD}^{\mathrm{b})}$ colorimetric method & 300 W Xe lamp & $\begin{array}{l}\mathrm{N}, \mathrm{N} \text {-diethyl-p-phenylenediamine, horseradish peroxidase: test solution phosphate } \\
\text { buffer solution }\left(0.5 \mathrm{M} \mathrm{KH}_{2} \mathrm{PO}_{4} \text { and } 0.5 \mathrm{M} \mathrm{K}_{2} \mathrm{HPO}_{4}, \mathrm{pH} \text { of } \sim 6.0\right) \text { with } 0.01 \mathrm{~g} / \mathrm{ml} \mathrm{DPD} \text { in } \\
0.1 \mathrm{M} \mathrm{H}_{2} \mathrm{SO}_{4} \text { solution and } 1 \mathrm{mg} / \mathrm{ml} \text { horseradish peroxidase; } \mathrm{H}_{2} \mathrm{O}_{2} \text { quantification at } \\
\lambda_{\max }=551 \mathrm{~nm} \text {. }\end{array}$ & [451] \\
\hline $\mathrm{SrTiO}_{3}$ & $\mathrm{O}_{2^{-}}-, \mathrm{OH} \bullet, \mathrm{O} \bullet$ & $\begin{array}{l}\text { Nanoscale redox titration } \\
\left(\mathrm{SECM}^{\mathrm{c})}, \mathrm{SI}-\mathrm{SECM}^{\mathrm{d})}\right)\end{array}$ & $300 \mathrm{~W}$ Xe lamp & $\begin{array}{l}\mathrm{SrTiO}_{3} \text { illumination (in the } 300 \mathrm{~nm} \text { to } 800 \mathrm{~nm} \text { range) in } 50 \mu \mathrm{M} \mathrm{K} \mathrm{K}_{3}\left[\mathrm{Fe}(\mathrm{CN})_{6}\right], 100 \mathrm{mM} \\
\text { borate buffer solution, } \mathrm{pH} 9.3 . \mathrm{SECM} \text { experiments - } 4 \text {-electrode configuration } \\
\left(\mathrm{SrTiO}_{3}\left(\mathrm{WE}^{\mathrm{e}}\right), \mathrm{Ag} / \mathrm{AgCl}(3 \mathrm{M} \mathrm{KCl})\left(\mathrm{RE}^{\mathrm{f}}\right), 1 \mathrm{~mm} \text { Pt wire }\left(\mathrm{CE}^{\mathrm{g})}\right) \text { with a } 4 \mu \mathrm{m} \text { radius }\right. \\
\text { carbon fiber ultramicroelectrode or a } 240 \mathrm{~nm} \text { radius pyrolyzed carbon } \\
\text { nanoelectrode. }\end{array}$ & {$[452,453]$} \\
\hline $\mathrm{W}-\mathrm{Mo} / \mathrm{BiVO}_{4}$ & $\mathrm{OH} \bullet$ & $\begin{array}{l}\text { Nanoscale redox titration } \\
\left(\mathrm{SECM}^{\mathrm{c})}, \mathrm{SI}-\mathrm{SECM}^{\mathrm{d})}\right)\end{array}$ & $300 \mathrm{~W}$ halogen bulb & $\begin{array}{l}\text { Redox couple } \mathrm{IrCl}_{6}^{2-/ 3-} \text { as a titrant, } \mathrm{Ag} / \mathrm{AgCl}(\text { sat. } \mathrm{KCl})(\mathrm{RE}), 1 \mathrm{Pt} \text { wire }(\mathrm{CE}) \text { using a } \\
4 \mu \mathrm{m} \text { radius carbon fiber ultramicroelectrode positioned at } 12 \mu \mathrm{m} \text { apart from the } \\
\text { working electrode in } 1 \mathrm{mM} \mathrm{K} \mathrm{K}_{2} \mathrm{ICl}_{6} \text { and } 0,1 \mathrm{M} \mathrm{Na}_{2} \mathrm{SO}_{4} \mathrm{pH} 4,5 \text {. }\end{array}$ & [454] \\
\hline $\mathrm{TiO}_{2}$ & $\mathrm{H}_{2} \mathrm{O}_{2}$ & SECM & 150 W Hg-Xe lamp & $\begin{array}{l}\mathrm{H}_{2} \mathrm{O}_{2} \text { microsensor - immobilizing horseradish peroxidase in an electron conducting } \\
\left.\left.\text { hydrogel based on poly(4-vinylpyridine) with [Os }(\mathrm{bpy})_{2}\right)_{2} \mathrm{Cl}\right]^{3+2+2+} \text { redox centers on } \\
\text { carbon fiber microelectrode. Independent monitoring of } \mathrm{H}_{2} \mathrm{O}_{2} \text { formation on } \mathrm{TiO}_{2} \\
\text { photoanode and ITO cathode in phosphate-buffered saline }(\mathrm{pH} \text { 7.4) using Pt wire } \\
\text { (CE) and a saturated calomel electrode (RE) at } 50 \mu \mathrm{m} \text { from the surface. }\end{array}$ & [455] \\
\hline $\mathrm{TiO}_{2}$ & $\mathrm{O}_{2^{0^{-}}}$ & $\begin{array}{l}\text { Method of chemiluminescent } \\
\text { probe }\end{array}$ & $150 \mathrm{~W}$ Xe arc lamp & $\begin{array}{l}\mathrm{TiO}_{2} \text { suspension }(4,3 \mathrm{mg} / \mathrm{ml} \text { in } 0,01 \mathrm{M} \mathrm{NaOH}, \mathrm{pH} 11) \text { with } \lambda_{\mathrm{exc}}=387 \text { or } 360 \mathrm{~nm} \text {, using } \\
\text { different glass filters with intensities } 40 \text { and } 3 \mathrm{~mW} / \mathrm{cm}^{2} \text {, respectively. } \\
\text { Pre-addition method - addition of } 7 \mathrm{mM} \text { luminol solution to } \mathrm{TiO}_{2} \text { suspension before } \\
\text { the irradiation; post-addition method - immediately after irradiation. }\end{array}$ & [456] \\
\hline $\mathrm{TiO}_{2}$ & $\begin{array}{l}\mathrm{OH} \cdot, \mathrm{O}_{2} \bullet^{-} \\
\mathrm{H}_{2} \mathrm{O}_{2}\end{array}$ & $\begin{array}{l}\text { Continuous flow } \\
\text { chemiluminescent analysis }\end{array}$ & $\begin{array}{l}500 \mathrm{~W} \text { xenon light } \\
\text { source }\end{array}$ & $\begin{array}{l}\mathrm{O}_{2}^{-} \text {detection } 0.1 \mathrm{mg} / \mathrm{mL} \mathrm{TiO}_{2} \text { suspension, } 1 \mu \mathrm{M} \text { luminol. } \\
\cdot \mathrm{OH} \text { detection , addition of phthalhydrazide }(1 \mu \mathrm{M}) \text { into the photoreactor }(\cdot \mathrm{OH} \\
\text { capture }) \text {, mixing with } \mathrm{H}_{2} \mathrm{O}_{2} / \mathrm{K}_{5} \mathrm{Cu}\left(\mathrm{HIO}_{6}\right)_{2}(0.1 \mathrm{mM}) \\
\mathrm{H}_{2} \mathrm{O}_{2} \text { detection, keeping } \mathrm{TiO}_{2} \text { suspension }(0,01 \mathrm{mg} / \mathrm{ml}) \text { in darkness for } 30 \mathrm{~min} \text {, and } \\
\text { mixing with luminol }(10 \mu \mathrm{M}) / \mathrm{K}_{3} \mathrm{Fe}(\mathrm{CN})_{6}(0,01 \mathrm{M}) \text {. }\end{array}$ & [457] \\
\hline 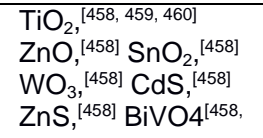 & $\begin{array}{l}\mathrm{OH} \cdot, \\
\mathrm{O}_{2} \cdot-\end{array}$ & $\begin{array}{l}\text { Photoluminescence }{ }^{[458]} \\
\text { fluorescence measurements }{ }^{[459]}\end{array}$ & $\begin{array}{l}350 \text { W Xe arc lamp }(365 \\
\text { nm) }{ }^{[458]} \\
200 \text { W high-pressure mercury } \\
\text { lamp }{ }^{[459]}\end{array}$ & 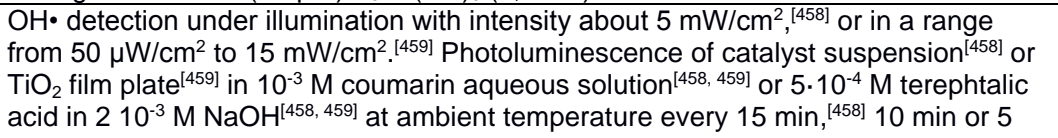 & [458, 460] \\
\hline
\end{tabular}




\begin{tabular}{|c|c|c|c|c|c|}
\hline $\mathrm{TiO}_{2} / \mathrm{CdS}$ & $\mathrm{H}_{2} \mathrm{~S}$ & Photoelectrochemical detection & $\begin{array}{l}500 \mathrm{~W} \text { Xe lamp } \\
(\lambda>420 \mathrm{~nm})\end{array}$ & $\begin{array}{l}\text { Three-electrode system }\left(\mathrm{TiO}_{2} / \mathrm{CdS} \text { film }(\mathrm{WE}), \mathrm{Ag} / \mathrm{AgCl}(\text { sat. } \mathrm{KCl})(\mathrm{RE}), \mathrm{Pt} \text { foil }(\mathrm{CE})\right) \\
\text { in } 0.5 \mathrm{M} \mathrm{LiNO}_{3} \text { solution containing } 10 \mathrm{mM} \mathrm{Fe}(\mathrm{CN})_{6}^{3-1 / 4}(\mathrm{pH}=6) \text {. }\end{array}$ & [461] \\
\hline $\mathrm{TiO}_{2}$ & $\mathrm{O}_{2}$ & $\begin{array}{l}\text { Photoelectrochemical detection, } \\
\text { photoluminescence } \\
\text { measurements }\end{array}$ & $\begin{array}{l}500 \mathrm{~W} \text { high-pressure mercury } \\
\text { lamp }(365 \mathrm{~nm})\end{array}$ & $\begin{array}{l}\text { Photocatalytic evolution of } \mathrm{O}_{2} \text { on } \mathrm{TiO}_{2} \text { rutile surfaces (WE) using in situ } \\
\text { measurements of photocurrent, photoluminescence and AFM using Pt plate (CE) } \\
\text { and } \mathrm{Ag} / \mathrm{AgCl} / \mathrm{KCl}(\mathrm{RE}) \text { in } 0.1 \mathrm{M} \mathrm{HClO}_{4} \text { solution. }\end{array}$ & [462] \\
\hline $\mathrm{TiO}_{2}$ & $\cdot \mathrm{OH},{ }^{1} \mathrm{O}_{2}$ & $\begin{array}{l}\text { Single molecule fluorescent } \\
\text { measurements }\end{array}$ & 100 W Hg lamp (365 nm) & $\begin{array}{l}\text { Detection of } \mathrm{OH} \cdot \text { diffused from the surface of pure } \mathrm{TiO}_{2} \text { to the } \mathrm{PMMA}^{\mathrm{i}} \text { glass } \\
\text { (intervening bandgap } 12.5 \mu \mathrm{m} \text { ) coated with } 3 \text {-(p-hydroxyphenyl)-fluorescein and } \\
\text { aminophenylfluorescein for } \mathrm{OH} \bullet \text { and terrilenediimide for }{ }^{1} \mathrm{O}_{2} \text { detection, respectively. }\end{array}$ & $\begin{array}{c}{[463,464,465,} \\
466]\end{array}$ \\
\hline $\begin{array}{l}\mathrm{TiO}_{2} / \mathrm{Ag} \\
\mathrm{TiO}_{2} / \mathrm{Ag} / \mathrm{Ni}\end{array}$ & $\mathrm{O}_{2^{\circ}}-$ & Photometric detection & $\begin{array}{l}125 \mathrm{~W} \text { high-pressure } \\
\text { mercury lamp }\end{array}$ & $\begin{array}{l}\text { Deactivation of gram-positive and gram-negative bacteria. } \\
\text { Illumination of samples under a thin layer of water with UV light for } 10 \mathrm{~min} \text {. } \\
\text { Immediately after illumination, addition of the tetranitromethane aqueous solution in } \\
\text { cuvette. Photometric detection of nitroform }\left(\lambda_{\max }=350 \mathrm{~nm}\right) \text {. }\end{array}$ & [383] \\
\hline $\mathrm{TiO}_{2}$ & $\mathrm{H}^{+}$ & SIET $^{\mathrm{j})}$ & UV LED ${ }^{\mathrm{k})}(365 \mathrm{~nm})$ & $\begin{array}{l}\text { Mapping of } \mathrm{H}^{+} \text {activity over } \mathrm{TiO}_{2} \text { surface in solution under irradiation using } \mathrm{pH} \\
\text { microelectrode. }\end{array}$ & {$[368,369,370]$} \\
\hline $\begin{array}{l}\mathrm{TiO}_{2},{ }^{[467]} \\
\mathrm{TiO}_{2} / \mathrm{Pd}{ }_{1}^{[468]} \\
\mathrm{BiO}^{[469]}\end{array}$ & NO & $\begin{array}{l}\text { Chemiluminescence } \mathrm{NO}_{x} \\
\text { detection }\end{array}$ & $\begin{array}{l}500 \mathrm{~W} \text { xenon arc lamp }{ }^{[467]} \\
\text { Solarsim } 150,[468] \text { LED lamp } \\
(448 \mathrm{~nm})^{[469]}\end{array}$ & $\begin{array}{l}\text { Irradiation of glass-fiber filters with } \mathrm{TiO}_{2} / \mathrm{Pd} \text { particles }{ }^{[468]} \text { or sample dish with } \mathrm{TiO}_{2} \\
\text { suspension }{ }^{[467]} \text { or } \mathrm{BiOl} \text { film on glass substrate }{ }^{[467]} \text { with solar light }(420-700 \mathrm{~nm}) \text { or } \\
\text { visible light }(448 \mathrm{~nm}),,^{[469]} \text { with flowing air stream at a rate } 3 \mathrm{~L} / \mathrm{min} \text {, }{ }^{[468]} 1.2 \mathrm{~L} / \mathrm{min},{ }^{[467]} \\
1 \mathrm{~L} / \mathrm{min}^{[469]} \text { containing } 1 \mathrm{ppm},{ }^{[468]} 400 \mathrm{ppb},{ }^{[467]} 600 \mathrm{ppb}^{[469]} \text { of } \mathrm{NO} \text {. }\end{array}$ & {$[467,468,469]$} \\
\hline $\mathrm{WO}_{3} / \mathrm{TiO}_{2}$ & $\mathrm{H}_{2} \mathrm{~S}, \mathrm{SO}_{2}$ & $\begin{array}{l}\text { Pulsed flamed photometric } \\
\text { detection }\end{array}$ & $8 \mathrm{~W}$ blacklight tube $(365 \mathrm{~nm})$ & $\begin{array}{l}\text { Irradiation of the reactor coated with } \mathrm{TiO}_{2} \text { aqueous suspension with flowing air } \\
\text { stream ( } 92 \text { vol. } \% \text { air, } 15 \mathrm{ppm} \mathrm{H} \mathrm{H}_{2} \mathrm{~S} \text { ) with flow rate } 200 \text { or } 500 \mathrm{~mL} / \mathrm{min}\end{array}$ & [470] \\
\hline CdS & $\begin{array}{l}\mathrm{HS}^{-}, \mathrm{S}_{2} \mathrm{O}_{3}^{2-} \\
\mathrm{SO}_{3}{ }^{2-}\end{array}$ & Spectrophotometric detection & $\begin{array}{l}1000 \text { W mercury high- } \\
\text { pressure lamp }\end{array}$ & $\begin{array}{l}\mathrm{HS} \text { detection: methylene blue }\left(\lambda_{\max }=670 \mathrm{~nm}, \varepsilon_{670}=24600 \mathrm{M}^{-1} \mathrm{~cm}^{-1}\right) \text { a product of } \mathrm{H}_{2} \mathrm{~S} \\
\text { and } \mathrm{N}, \mathrm{N} \text {-dimethyl-n-phenylene-diamine interaction } \\
\mathrm{SO}_{3}^{2-} \text { detection: formation of strongly colored compound }\left(\lambda_{\max }=590 \mathrm{~nm}\right) \text { in a reaction } \\
\text { between } \mathrm{SO}_{3}{ }^{2-} \text { and fuchsine in the presence of formaldehyde in aqueous-alcoholic } \\
\text { mixture } \\
\mathrm{S}_{2} \mathrm{O}_{3}{ }^{2-} \text { detection: reduction of } \mathrm{I}_{3}^{-}\left(\lambda_{\max }=360 \mathrm{~nm}, \varepsilon_{360}=24000 \mathrm{M}^{-1} \mathrm{~cm}^{-1}\right) \text { by sulfur(II-IV) } \\
\text { compounds - products of CdS photocorrosion }\end{array}$ & [471] \\
\hline
\end{tabular}

a) (UV-vis - ultraviolet-visible spectroscopy); ${ }^{\text {b) }}$ (DPD - N,N-diethyl-p-phenylenediamine); ${ }^{\text {c) }}$ (SECM - scanning electrochemical microscopy);

d) (SI-SECM - surface interrogation scanning electrochemical microscopy); ${ }^{\text {e) }}$ (WE - working electrode); ${ }^{\mathrm{f}}$ (RE - reference electrode); ${ }^{\mathrm{g})}$ (CE counter electrode); ${ }^{\text {h) }}$ (bpy - 2,2'-bipyridine); ${ }^{\text {i) }}$ (PMMA - polymethylmethacrylate); ${ }^{\text {j) }}$ (SIET - scanning ion-selective microelectrode technique);

k) (LED - light emitting diode) 


\section{References}

[1] H. Kroemer: Rev. Mod. Phys., 2001, 73, 783.

[2] D. Aspnes: Surf. Sci., 1994, 307, 1017.

[3] J. F. McGilp: Prog. Surf. Sci., 1995, 49, 1.

[4] J.-T. Zettler: Prog. Cryst. Growth Character., 1997, 35, 27.

[5] Z. Sobiesierski, D. I. Westwood, C. C. Matthai: J. Phys.: Cond. Mat., 1998, 10, 1.

[6] W. Richter: Appl. Phys. A, 2002, 75, 129.

[7] I. P. Herman: Ann. Rev. Phy. Chem., 2003, 54, 277.

[8] P. Weightman, D. S. Martin, R. J. Cole, T. Farrell: Rep. Prog. Phys., 2005, 68, 1251.

[9] D. V. Esposito, J. B. Baxter, J. John, N. S. Lewis, T. P. Moffat, T. Ogitsu, G. D. O’Neil, T. A. Pham, A. A. Talin, J. M. Velazquez, B. C. Wood: Energy Environ. Sci., 2015, 8, 2863.

[10] Q. Zhang, H. Li, L. Gan, Y. Ma, D. Golberg, T. Zhai: Chem. Soc. Rev., 2016, 45, 2694.

[11] M. Pristovsek: Habilitation thesis, Technische Universität Berlin (Germany), 2012.

[12] M. A. Green, K. Emery, Y. Hishikawa, W. Warta, E. D. Dunlop: Prog. Photovolt. Res. Applic., 2016, 24, 905.

[13] T. Tibbits, P. Beutel, M. Grave, C. Karcher, E. Oliva, G. Siefer, A. Wekkeli, M. Schachtner, F. Dimroth, A. Bett, R. Krause, M. Piccin, N. Blanc, M. Muñoz-Rico, C. Arena, E. Guiot, C. Charles-Alfred, C. Drazek, F. Janin, L. Farrugia, B. Hoarau, J. Wasselin, A. Tauzin, T. Signamarcheix, T. Hannappel, K. Schwarzburg,

A. Dobrich: Proc. EUPVSEC, 2014, 29, 1975.

[14] M. M. May, H.-J. Lewerenz, D. Lackner, F. Dimroth, T. Hannappel: Nat. Commun., 2015, 6, 8286.

[15] H. M. Manasevit, W. I. Simpson: J. Electrochem. Soc., 1969, 116, 1725.

[16] T. Hannappel, M. M. May, H.-J. Lewerenz: in H.-J. Lewerenz, L. Peter, editors, Photoelectrochemical Water Splitting: Materials, Processes and Architectures, pages 223-265, The Royal Society of Chemistry, ISBN 978-1-84973-647-3, 2013.

[17] O. Khaselev, J. A. Turner: Science, 1998, 280, 425.

[18] S. Hu, C. Xiang, S. Haussener, A. D. Berger, N. S. Lewis: Energy Environ. Sci., 2013, 6, 2984.

[19] H. Döscher, O. Supplie, M. M. May, P. Sippel, C. Heine, A. G. Muñoz, R. Eichberger, H.-J. Lewerenz, T. Hannappel: ChemPhysChem, 2012, 13, 2899.

[20] O. Supplie, M. M. May, H. Stange, C. Höhn, H.-J. Lewerenz, T. Hannappel: J. Appl. Phys., 2014, 115, 113509.

[21] U. Seidel, H.-J. Schimper, Z. Kollonitsch, K. Möller, K. Schwarzburg, T. Hannappel: J. Cryst. Growth, 2007, 298, 777.

[22] A. Dobrich: Dissertation thesis, Freie Universität Berlin, Germany, 2014.

[23] D. E. Aspnes, A. A. Studna: Phys. Rev. Lett., 1985, 54, 1956.

[24] T. Hannappel, S. Visbeck, L. Töben, F. Willig: Revi. Sci. Instrum., 2004, 75, 1297.

[25] A. Heller: Science, 1984, 223, 1141.

[26] A. G. Muñoz, C. Heine, M. Lublow, H. W. Klemm, N. Szabó, T. Hannappel, H.-J. Lewerenz: ECS J. Solid State Sci. Technol., 2013, 2, Q51.

[27] U. Seidel, B. Sagol, N. Szabó, K. Schwarzburg, T. Hannappel: Thin Solid Films, 2008, $516,6723$.

[28] B. E. Sagol, U. Seidel, N. Szabó, K. Schwarzburg, T. Hannappel: CHIMIA Internat. J. Chem., 2007, 61, 775.

[29] N. Szabó, B. E. Sagol, U. Seidel, K. Schwarzburg, T. Hannappel: phys. status solidi (RRL), 2008, 2, 254.

[30] F. Dimroth, M. Grave, P. Beutel, U. Fiedeler, C. Karcher, T. N. D. Tibbits, E. Oliva, G. Siefer, M. Schachtner, A. Wekkeli, A. W. Bett, R. Krause, M. Piccin, N. Blanc, 
C. Drazek, E. Guiot, B. Ghyselen, T. Salvetat, A. Tauzin, T. Signamarcheix,

A. Dobrich, T. Hannappel, K. Schwarzburg: , 2014, 22, 277.

[31] F. Dimroth, T. N. D. Tibbits, M. Niemeyer, F. Predan, P. Beutel, C. Karcher, E. Oliva, G. Siefer, D. Lackner, P. Fuß-Kailuweit, A. W. Bett, R. Krause, C. Drazek, E. Guiot, J. Wasselin, A. Tauzin, T. Signamarcheix: IEEE J. Photovolt., 2016, 6, 343.

[32] W. G. Schmidt, P. H. Hahn, F. Bechstedt, N. Esser, P. Vogt, A. Wange, W. Richter: Phys. Rev. Lett., 2003, 90, 126101.

[33] W. G. Schmidt, F. Bechstedt, N. Esser, M. Pristovsek, C. Schultz, W. Richter: Phys. Rev. B, 1998, 57, 14596.

[34] D. E. Aspnes, A. A. Studna: Phys. Rev. B, 1983, 27, 985.

[35] M. Zorn, T. Trepk, J.-T. Zettler, B. Junno, C. Meyne, K. Knorr, T. Wethkamp, M. Klein, M. Miller, W. Richter, L. Samuelson: Appl. Phys. A, 1997, 65, 333.

[36] W. G. Schmidt, N. Esser, A. M. Frisch, P. Vogt, J. Bernholc, F. Bechstedt, M. Zorn, T. Hannappel, S. Visbeck, F. Willig, W. Richter: Phys. Rev. B, 2000, 61, R16335.

[37] W. G. Schmidt, J. Bernholc, F. Bechstedt: Appl. Surf. Sci., 2000, 166, 179.

[38] T. Hannappel, L. Töben, S. Visbeck, H.-J. Crawack, C. Pettenkofer, F. Willig: Surf. Sci., 2000, 470, L1.

[39] N. Esser, W. G. Schmidt, C. Cobet, K. Fleischer, A. I. Shkrebtii, B. O. Fimland, W. Richter: J. Vac. Sci. Technol. B, 2001, 19, 1756.

[40] S. Visbeck, T. Hannappel, M. Zorn, J.-T. Zettler, F. Willig: Phys. Rev. B, 2001, 63, 245303.

[41] J. Luo, J. Geisz, J. Olson, M.-C. Su: J. Cryst. Growth, 1997, 174, 558.

[42] T. Hannappel, S. Visbeck, K. Knorr, J. Mahrt, M. Zorn, F. Willig: Appl. Phys. A, 1999, 69, 427.

[43] K. B. Ozanyan, P. J. Parbrook, M. Hopkinson, C. R. Whitehouse, Z. Sobiesierski, D. I. Westwood: J. Appl. Phys., 1997, 82, 474.

[44] P. Vogt, T. Hannappel, S. Visbeck, K. Knorr, N. Esser, W. Richter: Phys. Rev. B, 1999, $60, \mathrm{R} 5117$.

[45] P. Kleinschmidt, H. Döscher, P. Vogt, T. Hannappel: Phys. Rev. B, 2011, 83, 155316.

[46] L. Li, B.-K. Han, Q. Fu, R. F. Hicks: Phys. Rev. Lett., 1999, 82, 1879.

[47] T. Letzig, H.-J. Schimper, T. Hannappel, F. Willig: Phys. Rev. B, 2005, 71, 033308.

[48] T. Hannappel, S. Visbeck, M. Zorn, J.-T. Zettler, F. Willig: J. Cryst. Growth, 2000, 221, 124.

[49] M. M. May, O. Supplie, C. Höhn, W.-D. Zabka, H.-J. Lewerenz, R. van de Krol, T. Hannappel: Proc. SPIE, 2013, 8822, 88220M.

[50] M. M. May, H.-J. Lewerenz, T. Hannappel: J. Phys. Chem. C, 2014, 118, 19032.

[51] H. Döscher, K. Möller, T. Hannappel: J. Cryst. Growth, 2011, 318, 372.

[52] P. Lautenschlager, P. B. Allen, M. Cardona: Phys. Rev. B, 1985, 31, 2163.

[53] A. Frisch, P. Vogt, S. Visbeck, T. Hannappel, F. Willig, W. Braun, W. Richter, J. Bernholc, W. Schmidt, N. Esser: Appl. Surf. Sci., 2000, 166, 224.

[54] L. Töben, T. Hannappel, R. Eichberger, K. Möller, L. Gundlach, R. Ernstorfer, F. Willig: J. Crys. Growth, 2003, 248, 206.

[55] L. Töben, L. Gundlach, R. Ernstorfer, R. Eichberger, T. Hannappel, F. Willig, A. Zeiser, J. Förstner, A. Knorr, P. H. Hahn, W. G. Schmidt: Phys. Rev. Lett., 2005, 94, 067601.

[56] L. Töben, L. Gundlach, T. Hannappel, R. Ernstorfer, R. Eichberger, F. Willig: Appl. Phys. A, 2004, 78, 239.

[57] P. Sippel, J. M. Szarko, T. Hannappel, R. Eichberger: Phys. Rev. B, 2015, 91, 115312.

[58] M. Arens, P. Kurpas, P. Ressel, M. Weyers: Thin Solid Films, 1998, 313-314, 609.

[59] B. Kaiser, D. Fertig, J. Ziegler, J. Klett, S. Hoch, W. Jaegermann: ChemPhysChem, 2012, 13, 3053. 
[60] M. Feifel, T. Rachow, J. Benick, J. Ohlmann, S. Janz, M. Hermle, F. Dimroth, D. Lackner: IEEE J. Photovolt., 2016, 6, 384.

[61] J. Geisz, J. Olson, D. Friedman, K. Jones, R. Reedy, M. Romero: Proc. IEEE PVSC, 2005, 31, 695.

[62] N. Esser, W. Schmidt, J. Bernholc, A. Frisch, P. Vogt, M. Zorn, M. Pristovsek, W. Richter, F. Bechstedt, T. Hannappel, S. Visbeck: J. Vac. Sci. Technol. B, 1999, 17, 1691.

[63] L. Töben, T. Hannappel, K. Möller, H. Crawack, C. Pettenkofer, F. Willig: Surf. Sci., 2001, 494, L755.

[64] A. M. Frisch, W. G. Schmidt, J. Bernholc, M. Pristovsek, N. Esser, W. Richter: Phys. Rev. B, 1999, 60, 2488.

[65] P. Sippel, O. Supplie, M. M. May, R. Eichberger, T. Hannappel: Phys. Rev. B, 2014, $89,165312$.

[66] P. H. Hahn, W. G. Schmidt, F. Bechstedt, O. Pulci, R. del Sole: Phys. Rev. B, 2003, 68, 033311.

[67] N. Kadotani, M. Shimomura, Y. Fukuda: Phys. Rev. B, 2004, 70, 165323.

[68] H. Döscher, T. Hannappel: J. Appl. Phys., 2010, 107, 123523.

[69] B. M. Kayes, H. Nie, R. Twist, S. G. Spruytte, F. Reinhardt, I. C. Kizilyalli, G. S. Higashi: Proc. IEEE PVSC, 2011, 37, 000004.

[70] L. S. Mattos, S. R. Scully, M. Syfu, E. Olson, L. Yang, C. Ling, B. M. Kayes, G. He: Proc. IEEE PVSC, 2012, 38, 003187.

[71] T. Takamoto, M. Kaneiwa, M. Imaizumi, M. Yamaguchi: Prog. Photovolt. Res. Applic., 2005, 13, 495.

[72] J. M. Olson, S. R. Kurtz, A. E. Kibbler, P. Faine: Appl. Phys. Lett., 1990, 56, 623.

[73] A. Luque: J. Appl. Phys., 2011, 110, 031301.

[74] C. Fetzer, B. Jun, K. Edmondson, S. Khemthong, K. Rouhani, R. Cravens, R. Bardfield, M. Gillanders: Proc. IEEE PVSC, 2008, 33, 1.

[75] W. Guter, J. Schöne, S. P. Philipps, M. Steiner, G. Siefer, A. Wekkeli, E. Welser, E. Oliva, A. W. Bett, F. Dimroth: Appl. Phys. Lett., 2009, 94, 223504.

[76] R. M. France, F. Dimroth, T. J. Grassman, R. R. King: MRS Bulletin, 2016, 41, 202.

[77] D. E. Aspnes, E. Colas, A. A. Studna, R. Bhat, M. A. Koza, V. G. Keramidas: Phys. Rev. Lett., 1988, 61, 2782.

[78] J. P. Harbison, D. E. Aspnes, A. A. Studna, L. T. Florez, M. K. Kelly: Appl. Phys. Lett., 1988, 52, 2046.

[79] D. Aspnes: Mater. Sci. Eng. B, 1995, 30, 109.

[80] A. Balzarotti, M. Fanfoni, F. Patella, F. Arciprete, E. Placidi, G. Onida, R. D. Sole: Surf. Sci. Lett., 2003, 524, L71.

[81] T. Makimoto, Y. Yamauchi, N. Kobayashi, Y. Horikoshi: Jap. J. Appl. Phys., 1990, 29, L645.

[82] I. Kamiya, D. E. Aspnes, H. Tanaka, L. T. Florez, J. P. Harbison, R. Bhat: Phys. Rev. Lett., 1992, 68, 627.

[83] D. E. Aspnes, I. Kamiya, H. Tanaka, R. Bhat: J. Vac. Sci. Technol. B, 1992, 10, 1725.

[84] I. Kamiya, H. Tanaka, D. E. Aspnes, M. Koza, R. Bhat: Appl. Phys. Lett., 1993, 63, 3206.

[85] K. Ploska, J.-T. Zettler, W. Richter, J. Jönsson, F. Reinhardt, J. Rumberg, M. Pristovsek, M. Zorn, D. Westwood, R. Williams: J. Cryst. Growth, 1994, 145, 44.

[86] K. Deppert, J. Jönsson, L. Samuelson: Thin Solid Films, 1993, 224, 133.

[87] M. Deura, M. Sugiyama, T. Nakano, Y. Nakano, Y. Shimogaki: Jap. J. Appl. Phys., 2007, 46, 6519.

[88] J. Creighton, K. Baucom: Surf. Sci., 1998, 409, 372.

[89] S.-H. Lee, W. Moritz, M. Scheffler: Phys. Rev. Lett., 2000, 85, 3890. 
[90] W. G. Schmidt, S. Mirbt, F. Bechstedt: Phys. Rev. B, 2000, 62, 8087.

[91] W. Schmidt, F. Bechstedt, K. Fleischer, C. Cobet, N. Esser, W. Richter, J. Bernholc, G. Onida: phys. status solidi (a), 2001, 188, 1401.

[92] I. Kamiya, D. E. Aspnes, L. T. Florez, J. P. Harbison: Phys. Rev. B, 1992, 46, 15894.

[93] M. Wassermeier, I. Kamiya, D. E. Aspnes, L. T. Florez, J. P. Harbison, P. M. Petroff: J. Vac. Sci. Technol. B, 1991, 9, 2263.

[94] D. E. Aspnes, Y. C. Chang, A. A. Studna, L. T. Florez, H. H. Farrell, J. P. Harbison: Phys. Rev. Lett., 1990, 64, 192.

[95] U. Resch, S. Scholz, U. Rossow, A. Müller, W. Richter, A. Förster: Appl. Surf. Sci., 1993, 63, 106.

[96] M. J. Begarney, L. Li, C. H. Li, D. C. Law, Q. Fu, R. F. Hicks: Phys. Rev. B, 2000, 62, 8092.

[97] S. J. Morris, J. M. Bass, C. C. Matthai: Phys. Rev. B, 1995, 52, 16739.

[98] A. I. Shkrebtii, N. Esser, W. Richter, W. G. Schmidt, F. Bechstedt, B. O. Fimland, A. Kley, R. Del Sole: Phys. Rev. Lett., 1998, 81, 721.

[99] L. F. Lastras-Martnez, D. Rönnow, P. V. Santos, M. Cardona, K. Eberl: Phys. Rev. B, 2001, 64, 245303.

[100] L. F. Lastras-Martnez, J. M. Flores-Camacho, R. E. Balderas-Navarro, M. ChaviraRodrguez, A. Lastras-Martnez, M. Cardona: Phys. Rev. B, 2007, 75, 235315.

[101] W. G. Schmidt, F. Bechstedt, W. Lu, J. Bernholc: Phys. Rev. B, 2002, 66, 085334.

[102] F. Arciprete, C. Goletti, E. Placidi, C. Hogan, P. Chiaradia, M. Fanfoni, F. Patella, A. Balzarotti: Phys. Rev. B, 2004, 69, 081308.

[103] C. Goletti, V. Emiliani, S. Schintke, A. M. Frisch, N. Esser, B. O. Fimland: phys. status solidi (b), 2005, 242, 2664.

[104] D. Paget, C. Hogan, V. L. Berkovits, O. E. Tereshchenko: Phys. Rev. B, 2003, 67, 245313.

[105] F. Arciprete, C. Goletti, E. Placidi, P. Chiaradia, M. Fanfoni, F. Patella, C. Hogan, A. Balzarotti: Phys. Rev. B, 2003, 68, 125328.

[106] R. Onitsuka, M. Sugiyama, Y. Nakano: J. Cryst. Growth, 2010, 312, 1343.

[107] P. Kurpas, J. Jönsson, W. Richter, D. Gutsche, M. Pristovsek, M. Zorn: Jo. Cryst. Growth, 1994, 145, 36.

[108] S. E. Acosta-Ortiz, A. Lastras-Martínez: Phys. Rev. B, 1989, 40, 1426.

[109] Z. Sobiesierski, D. I. Westwood, M. Elliott: Phys. Rev. B, 1997, 56, 15277.

[110] H. Tanaka, E. Colas, I. Kamiya, D. E. Aspnes, R. Bhat: Appl. Phys. Lett., 1991, 59, 3443.

[111] M. Pristovsek, S. Tsukamoto, N. Koguchi, B. Han, K. Haberland, J.-T. Zettler, W. Richter, M. Zorn, M. Weyers: phys. status solidi (a), 2001, 188, 1423.

[112] M. Pristovsek, S. Tsukamoto, B. Han, J.-T. Zettler, W. Richter: J. Cryst. Growth, 2003, 248, 254.

[113] M. Pristovsek, B. Han, J.-T. Zettler, W. Richter: J. Cryst. Growth, 2000, 221, 149 , proc Tenth Int Conf Metalorganic Vapor Phase Epitaxy.

[114] M. Wassermeier, J. Behrend, J.-T. Zettler, K. Stahrenberg, K. Ploog: Appl. Surf. Sci., 1996, 107, 48.

[115] Z. A. Ibrahim, A. I. Shkrebtii, M. J. G. Lee, K. Vynck, T. Teatro, W. Richter, T. Trepk, T. Zettler: Phys. Rev. B, 2008, 77, 125218.

[116] B. Rähmer, M. Pristovsek, M. Breusing, R. Kremzow, W. Richter: Appl. Phys. Lett., 2006, 89, 063108 .

[117] R. Kremzow, M. Pristovsek, M. Kneissl: J. Cryst. Growth, 2008, 310, 4751.

[118] M. Pristovsek, R. Kremzow, M. Kneissl: Jap. J. Appl. Phys., 2013, 52, 041201. 
[119] R. R. King, D. Bhusari, D. Larrabee, X.-Q. Liu, E. Rehder, K. Edmondson, H. Cotal, R. K. Jones, J. H. Ermer, C. M. Fetzer, D. C. Law, N. H. Karam: Prog. Photovolt. Res. Applic., 2012, 20, 801.

[120] J. Gupta, S. Watkins, R. Arés, G. Soerensen: J. Cryst. Growth, 1998, 195, 205.

[121] M. Deura, Y. Shimogaki, Y. Nakano, M. Sugiyama: J. Cryst. Growth, 2008, 310, 4736.

[122] W. Richter, J.-T. Zettler: Appl. Surf. Sci., 1996, 100, 465.

[123] P. Wolfram, E. Steimetz, W. Ebert, B. Henninger, J.-T. Zettler: J. Cryst. Growth, 2003, $248,240$.

[124] N. A. Kalyuzhnyy, V. V. Evstropov, V. M. Lantratov, S. A. Mintairov, M. A.

Mintairov, A. S. Gudovskikh, A. Luque, V. M. Andreev: Internat. J. Photoenergy, 2014, 836284.

[125] M. Zorn, P. Kurpas, A. I. Shkrebtii, B. Junno, A. Bhattacharya, K. Knorr, M. Weyers, L. Samuelson, J. T. Zettler, W. Richter: Phys. Rev. B, 1999, 60, 8185.

[126] C. Krahmer, M. Philippens, M. Schubert, K. Streubel: J. Cryst. Growth, 2007, 298, 18.

[127] M. Zorn, J. Jönsson, A. Krost, W. Richter, J.-T. Zettler, K. Ploska, F. Reinhardt: J. Cryst. Growth, 1994, 145, 53.

[128] S. M. Scholz, A. B. Müller, W. Richter, D. R. T. Zahn, D. I. Westwood, D. A. Woolf, R. H. Williams: J. Vac. Sci. Technol. B, 1992, 10, 1710.

[129] A. Kussmaul, S. Vernon, P. C. Colter, R. Sudharsanan, A. Mastrovito, K. J. Linden, N. H. Karam, N. H. Karam, S. C. Warnick, M. A. Dahleh: J. Electron. Mater., 1997, $26,1145$.

[130] P. Kurpas, A. Oster, M. Weyers, A. Rumberg, K. Knorr, W. Richter: J. Electron. Mater., 1997, 26, 1159.

[131] F. Bugge, M. Zorn, U. Zeimer, A. Pietrzak, G. Erbert, M. Weyers: J. Cryst. Growth, 2009, 311, 1065.

[132] M. Zorn, M. Weyers: J. Cryst. Growth, 2005, 276, 29.

[133] T. Trepk, M. Zorn, J.-T. Zettler, M. Klein, W. Richter: Thin Solid Films, 1998, 313â€"314, 496.

[134] J.-S. Lee, Y. Masumoto: J. Cryst. Growth, 2000, 221, 111.

[135] K. Haberland, A. Kaluza, M. Zorn, M. Pristovsek, H. Hardtdegen, M. Weyers, J.-T. Zettler, W. Richter: J. Cryst. Growth, 2002, 240, 87.

[136] S. J. Morris, J.-T. Zettler, K. C. Rose, D. I. Westwood, D. A. Woolf, R. H. Williams, W. Richter: J. Appl. Phys., 1995, 77, 3115.

[137] K. Haberland, A. Bhattacharya, M. Zorn, M. Weyers, J. T. Zettler, W. Richter: J. Electron. Mater., 2000, 29, 468.

[138] P. Wolfram, E. Steimetz, W. Ebert, N. Grote, J.-T. Zettler: J. Cryst. Growth, 2004, 272, 118.

[139] M. Zorn, J.-T. Zettler: phys. status solidi (b), 2005, 242, 2587.

[140] M. Zorn, J.-T. Zettler, A. Knauer, M. Weyers: J. Cryst. Growth, 2006, 287, 637.

[141] R. Ehlert, F. Poser, N. Esser, P. Vogt, W. Richter: phys. status solidi (b), 2005, 242, 2575.

[142] O. Reentilä, M. Mattila, M. Sopanen, H. Lipsanen: J. Appl. Phys., 2007, 101, 033533.

[143] T. Tsuchiya, T. Taniwatari, K. Uomi, T. Kawano, Y. Ono: Jap. J. Appl. Phys., 1993, $32,4652$.

[144] M. Heurlin, N. Anttu, C. Camus, L. Samuelson, M. T. Borgström: Nano Lett., 2015, 15, 3597.

[145] C. Prall, C. Kaspari, F. Brunner, K. Haberland, M. Weyers, D. Rueter: J. Cryst. Growth, 2015, 415, 1.

[146] C. Prall, M. Ruebesam, C. Weber, M. Reufer, D. Rueter: J. Cryst. Growth, 2014, 397, 24.

[147] J.-S. Lee, S. Sugou, Y. Masumoto: J. Appl. Phys., 2000, 88, 196. 
[148] F. Dimroth, T. Roesener, S. Essig, C. Weuffen, A. Wekkeli, E. Oliva, G. Siefer, K. Volz, T. Hannappel, D. Häussler, W. Jäger, A. W. Bett: IEEE J. Photovolt., 2014, 4, 620.

[149] T. Roesener, H. Döscher, A. Beyer, S. Brückner, V. Klinger, A. Wekkeli, P. Kleinschmidt, C. Jurecka, J. Ohlmann, K. Volz, W. Stolz, T. Hannappel, A. W. Bett, F. Dimroth: Proc. EU PVSEC, 2010, 25, 964.

[150] T. J. Grassman, D. J. Chmielewski, S. D. Carnevale, J. A. Carlin, S. A. Ringel: IEEE J. Photovolt., 2016, 6, 326.

[151] S. Almosni, C. Robert, T. Nguyen Thanh, C. Cornet, A. Létoublon, T. Quinci, C. Levallois, M. Perrin, J. Kuyyalil, L. Pedesseau, A. Balocchi, P. Barate, J. Even, J. M. Jancu, N. Bertru, X. Marie, O. Durand, A. Le Corre: J. Appl. Phys., 2013, 113, 123509.

[152] A. Rolland, L. Pedesseau, J. Even, S. Almosni, C. Robert, C. Cornet, J. M. Jancu, J. Benhlal, O. Durand, A. L. Corre, P. Rale, L. Lombez, J.-F. Guillemoles, E. Tea, S. Laribi: Opt. Quant. Electron., 2014, 46, 1397.

[153] S. R. Kurtz, P. Faine, J. M. Olson: J. Appl. Phys., 1990, 68, 1890.

[154] J. A. del Alamo: Nature, 2011, 479, 317.

[155] H. Kroemer: J. Cryst. Growth, 1987, 81, 193.

[156] S. F. Fang, K. Adomi, S. Iyer, H. Morkoc, H. Zabel, C. Choi, N. Otsuka: J. Appl. Phys., 1990, 68, R31.

[157] D. J. Chadi: Phys. Rev. Lett., 1987, 59, 1691.

[158] R. E. Schlier, H. E. Farnsworth: J. Chem. Phys., 1959, 30, 917.

[159] R. M. Tromp, R. J. Hamers, J. E. Demuth: Phys. Rev. Lett., 1985, 55, 1303.

[160] R. A. Wolkow: Phys. Rev. Lett., 1992, 68, 2636.

[161] D. E. Aspnes, J. Ihm: Phys. Rev. Lett., 1986, 57, 3054.

[162] Y. J. Chabal, K. Raghavachari: Phys. Rev. Lett., 1984, 53, 282.

[163] T. W. Poon, S. Yip, P. S. Ho, F. F. Abraham: Phys. Rev. Lett., 1990, 65, 2161.

[164] B. S. Swartzentruber, Y.-W. Mo, M. B. Webb, M. G. Lagally: J. Vac. Sci. Technol. A, 1989, 7, 2901.

[165] P. Bedrossian, T. Klitsner: Phys. Rev. Lett., 1992, 68, 646.

[166] T. Doi, M. Ichikawa, S. Hosoki, K. Ninomiya: Phys. Rev. B, 1996, 53, 16609.

[167] F. K. Men, W. E. Packard, M. B. Webb: Phys. Rev. Lett., 1988, 61, 2469.

[168] B. S. Swartzentruber, C. M. Matzke, D. L. Kendall, J. E. Houston: Surf. Sci., 1995, $329,83$.

[169] J. J. Boland: Phys. Rev. Lett., 1990, 65, 3325.

[170] J. E. Northrup: Phys. Rev. B, 1991, 44, 1419.

[171] T. Aoyama, K.-i. Goto, T. Yamazaki, T. Ito: J. Vac. Sci. Technol. A, 1996, 14, 2909.

[172] H. Bender, S. Verhaverbeke, M. Caymax, O. Vatel, M. M. Heyns: J. Appl. Phys., 1994, $75,1207$.

[173] K. W. Kolasinski, W. Nessler, A. de Meijere, E. Hasselbrink: Phys. Rev. Lett., 1994, $72,1356$.

[174] T. Komeda, Y. Kumagai: Phys. Rev. B, 1998, 58, 1385.

[175] A. Dobrich, P. Kleinschmidt, H. Döscher, T. Hannappel: J. Vac. Sci. Technol. B, 2011, 29, 04D114.

[176] H. Döscher, A. Dobrich, S. Brückner, P. Kleinschmidt, T. Hannappel: Appl. Phys. Lett., 2010, 97, 151905.

[177] Y. J. Chabal, A. L. Harris, K. Raghavachari, J. C. Tully: Int. J. Mod. Phys. B, 1993, 7, 1031.

[178] Y. Chabal: Surf. Sci., 1986, 168, 594.

[179] S. Brückner, H. Döscher, P. Kleinschmidt, T. Hannappel: Appl. Phys. Lett., 2011, 98, 211909. 
[180] P. Rosenow, R. Tonner: J. Chem. Phys., 2016, 144, 204706.

[181] S. Brückner, O. Supplie, A. Dobrich, A. Paszuk, P. Kleinschmidt, T. Hannappel:, 2017, submitted.

[182] S. Jeong, A. Oshiyama: Phys. Rev. Lett., 1998, 81, 5366.

[183] F. A. Reboredo, S. B. Zhang, A. Zunger: Phys. Rev. B, 2001, 63, 125316.

[184] A. R. Laracuente, L. J. Whitman: Surf. Sci., 2003, 545, 70.

[185] K. Kitahara, O. Ueda: Jap. J. Appl. Phys., 1994, 33, L1571.

[186] J. M. Olson, M. M. Al-Jassim, A. Kibbler, K. M. Jones: J. Cryst. Growth, 1986, 77, 515.

[187] B. Kunert, I. Németh, S. Reinhard, K. Volz, W. Stolz: Thin Solid Films, 2008, 517, 140.

[188] J. A. Appelbaum, G. A. Baraff, D. R. Hamann: Phys. Rev. B, 1976, 14, 588.

[189] K. Hingerl, R. E. Balderas-Navarro, A. Bonanni, P. Tichopadek, W. G. Schmidt: Appl. Surf. Sci., 2001, 175-176, 769.

[190] W. G. Schmidt, F. Bechstedt, J. Bernholc: Phys. Rev. B, 2001, 63, 045322.

[191] M. Palummo, N. Witkowski, O. Pluchery, R. del Sole, Y. Borensztein: Phys. Rev. B, 2009, 79, 035327.

[192] R. Shioda, J. van der Weide: Appl. Surf. Sci., 1998, 130-132, 266.

[193] S. Brückner, H. Döscher, P. Kleinschmidt, O. Supplie, A. Dobrich, T. Hannappel: Phys. Rev. B, 2012, 86, 195310.

[194] H. Döscher, S. Brückner, A. Dobrich, C. Höhn, P. Kleinschmidt, T. Hannappel: J. Cryst. Growth, 2011, 315, 10.

[195] Z. Zhang, H. Chen, B. C. Bolding, M. G. Lagally: Phys. Rev. Lett., 1993, 71, 3677.

[196] S. Brückner, P. Kleinschmidt, O. Supplie, H. Döscher, T. Hannappel: New J. Phys., 2013, 15, 113049 .

[197] B. M. Gallois, T. M. Besmann, M. W. Stott: J. Amer. Ceram. Soc., 1994, 77, 2949.

[198] O. Supplie, S. Brückner, O. Romanyuk, H. Döscher, C. Höhn, M. M. May, P. Kleinschmidt, F. Grosse, T. Hannappel: Phys. Rev. B, 2014, 90, 235301.

[199] H. Döscher, S. Brückner, T. Hannappel: J. Cryst. Growth, 2011, 318, 563.

[200] T. Hannappel, W. E. McMahon, J. M. Olson: J. Cryst. Growth, 2004, 272, 24.

[201] W. E. McMahon, E. L. Warren, A. E. Kibbler, R. M. France, A. G. Norman, R. C. Reedy, J. M. Olson, A. C. Tamboli, P. Stradins: J. Cryst. Growth, 2016, in print.

[202] A. Paszuk, A. Dobrich, C. Koppka, S. Brückner, M. Duda, P. Kleinschmidt, O. Supplie, T. Hannappel: J. Cryst. Growth, 2017, 464, 8.

[203] E. García-Tabarés, D. Martín, I. García, J. F. Leliévre, I. Rey-Stolle: AIP Conf. Proc., 2012, 1477, 5.

[204] Y. Kohama, K. Uchida, T. Soga, T. Jimbo, M. Umeno: Appl. Phys. Lett., 1988, 53, 862.

[205] R. D. Bringans, D. Biegelsen, L.-E. Swartz: Phys. Rev. B, 1991, 44, 3054.

[206] L. Kipp, D. Biegelsen, J. Northrup, L.-E. Swartz, R. D. Bringans: Phys. Rev. Lett., 1996, 76, 2810.

[207] T. Bork, W. McMahon, J. Olson, T. Hannappel: J. Cryst. Growth, 2007, 298, 54.

[208] O. Supplie, M. M. May, P. Kleinschmidt, A. Nägelein, A. Paszuk, S. Brückner, T. Hannappel: APL Mater., 2015, 3, 126110.

[209] E. L. Warren, A. E. Kibbler, R. M. France, A. G. Norman, P. Stradins, W. E. McMahon: Appl. Phys. Lett., 2015, 107, 082109.

[210] W. McMahon, I. Batyrev, T. Hannappel, J. Olson, S. Zhang: Phys. Rev. B, 2006, 74, 033304.

[211] H. J. Zandvliet: Phys. Rep., 2003, 388, 1.

[212] T. Yasuda, L. Mantese, U. Rossow, D. E. Aspnes: Phys. Rev. Lett., 1995, 74, 3431.

[213] U. Rossow, L. Mantese, D. E. Aspnes: J. Vac. Sci. Technol. B, 2000, 18, 2229. 
[214] S. Brückner, E. Barrigón, O. Supplie, P. Kleinschmidt, A. Dobrich, C. Löbbel, I. ReyStolle, H. Döscher, T. Hannappel: phys. status solidi $(R R L), \mathbf{2 0 1 2}, 6,178$.

[215] E. Barrigón, S. Brückner, O. Supplie, P. Kleinschmidt, I. Rey-Stolle, T. Hannappel: Appl. Phys. Lett., 2013, 102, 111608.

[216] S. Banerjee, J. F. McGilp, C. H. Patterson: phys. status solidi (b), 2015, 252, 78.

[217] E. Barrigon, B. Galiana, I. Rey-Stolle: J. Cryst. Growth, 2011, 315, 22.

[218] H. Döscher, T. Hannappel, B. Kunert, A. Beyer, K. Volz, W. Stolz: Appl. Phys. Lett., 2008, 93, 172110.

[219] W. He, S. L. Lu, J. R. Dong, Y. M. Zhao, X. Y. Ren, K. L. Xiong, B. Li, H. Yang, H. M. Zhu, X. Y. Chen, X. Kong: Appl. Phys. Lett., 2010, 97, 121909.

[220] K. Volz, A. Beyer, W. Witte, J. Ohlmann, I. Németh, B. Kunert, W. Stolz: J. Cryst. Growth, 2011, 315, 37.

[221] B. Galiana, E. Barrigón, I. Rey-Stolle, V. Corregidor, P. Espinet, C. Algora, E. Alves: Superlatt. Microstruct., 2009, 45, 277.

[222] Y. Li, L. Lazzarini, L. J. Giling, G. Salviati: J. Appl. Phys., 1994, 76, 57483.

[223] S. M. Ting, E. A. Fitzgerald: J. Appl. Phys., 2000, 87, 2618.

[224] H. W. Yu, E. Y. Chang, Y. Yamamoto, B. Tillack, W. C. Wang, C. I. Kuo, Y. Y. Wong, H. Q. Nguyen: Appl. Phys. Lett., 2011, 99, 171908.

[225] B. Galiana, I. Rey-Stolle, I. Beinik, C. Algora, C. Teichert, J. Molina-Aldareguia, P. Tejedor: Sol. Energ. Mat. Sol. Cells, 2011, 95, 1949.

[226] W. E. McMahon, J. M. Olson: Phys. Rev. B, 1999, 60, 15999.

[227] W. E. McMahon, J. M. Olson: Phys. Rev. B, 1999, 60, 2480.

[228] S. Gan, L. Li, M. J. Begarney, D. Law, B.-K. Han, R. F. Hicks: J. Appl. Phys., 1999, 85, 2004.

[229] S. M. Ting, E. A. Fitzgerald: J. Appl. Phys., 2000, 87, 2618.

[230] S. Brückner, O. Supplie, E. Barrigón, J. Luczak, P. Kleinschmidt, I. Rey-Stolle, H. Döscher, T. Hannappel: Appl. Phys. Lett., 2012, 101, 121602.

[231] W. McMahon, A. Kibbler, J. Olson: Surf. Sci, 2004, 571, 146.

[232] Y. Shimamune, M. Sakuraba, T. Matsuura, J. Murota: Appl. Surf. Sci., 2000, 162-163, 390.

[233] W. McMahon, J. Olson: J. Cryst. Growth, 2001, 225, 410, proceedings of the 12th American Conference on Crystal Growth and Epitaxy.

[234] M. Zorn, J. Jönsson, W. Richter, J.-T. Zettler, K. Ploska: phys. status solidi (a), 1995, $152,23$.

[235] K. Haberland, M. Zorn, A. Klein, A. Bhattacharya, M. Weyers, J.-T. Zettler, W. Richter: J. Cryst. Growth, 2003, 248, 194.

[236] N. Dietz, U. Rossow, D. E. Aspnes, K. J. Bachmann: J. Cryst. Growth, 1996, 164, 34.

[237] U. Rossow, N. Dietz, K. J. Bachmann, D. E. Aspnes: J. Vac. Sci. Technol. B, 1996, 14, 3040 .

[238] T. Yasuda: Thin Solid Films, 1998, 313-314, 544.

[239] T. Yasuda, K. Kimura, S. Miwa, L. H. Kuo, C. G. Jin, K. Tanaka, T. Yao: Phys. Rev. Lett., 1996, 77, 326.

[240] T. Yasuda, L. H. Kuo, K. Kimura, S. Miwa, C. G. Jin, K. Tanaka, T. Yao: J. Vac. Sci. Technol. B, 1996, 14, 3052 .

[241] T. Yasuda, D. E. Aspnes, D. R. Lee, C. H. Bjorkman, G. Lucovsky: J. Vac. Sci. Technol. A, 1994, 12, 1152.

[242] O. Hunderi, J.-T. Zettler, K. Haberland: Thin Solid Films, 2005, 472, 261.

[243] O. Supplie, T. Hannappel, M. Pristovsek, H. Döscher: Phys. Rev. B, 2012, 86, 035308.

[244] M. Sugiyama, K. Sugita, Y. Wang, Y. Nakano: J. Cryst. Growth, 2011, 315, 1.

[245] H. Sodabanlu, S. Ma, K. Watanabe, M. Sugiyama, Y. Nakano: Appl. Phys. Express, 2012, 5, 062301. 
[246] S. Sudo, Y. Nakano, M. Sugiyama, Y. Shimogaki, H. Komiyama, K. Tada: Proc. IPRM, 1998, page 485.

[247] Y. Wang, R. Onitsuka, M. Deura, W. Yu, M. Sugiyama, Y. Nakano: J. Cryst. Growth, 2010, 312, 1364.

[248] P. Würfel: Physics of solar cells, Wiley-VCH, 2009, ISBN 978-3-527-40857-3.

[249] R. Brendel, J. H. Werner, H. J. Queisser: Sol. Energ. Mat. Sol. Cells, 1996, 41, 419.

[250] S. Ma, H. Sodabanlu, K. Watanabe, M. Sugiyama, Y. Nakano: Jap. J. Appl. Phys., 2012, 51, 10ND09.

[251] J. Decobert, G. Patriarche: J. Appl. Phys., 2002, 92, 5749.

[252] N. L. Rowell, G. Yu, D. J. Lockwood, P. J. Poole: J. Vac. Sci. Technol. A, 2004, 22, 897.

[253] J. Camassel, J. Laurenti, S. Juillaguet, F. Reinhardt, K. Wolter, H. Kurz, D. Grützmacher: J. Cryst. Growth, 1991, 107, 543.

[254] A. Dobrich, K. Schwarzburg, T. Hannappel: Sol. Energ. Mat. Sol. Cells, 2016, 148, 25.

[255] H. Döscher, P. Hens, A. Beyer, L. Tapfer, K. Volz, W. Stolz: J. Crys. Growth, 2016, in press, doi: 10.1016/j.jcrysgro.2016.10.055.

[256] U. Seidel, T. Hannappel: J. Cryst. Growth, 2008, 310, 2334.

[257] U. Seidel, E. Sagol, C. Pettenkofer, T. Hannappel: Appl. Surf. Sci., 2008, 255, 722.

[258] O. Pitts, S. Watkins, C. Wang, V. Fink, K. Kavanagh: J. Cryst. Growth, 2003, 254, 28.

[259] O. Pitts, S. Watkins, C. Wang: J. Cryst. Growth, 2003, 248, 249.

[260] O. Supplie, M. M. May, C. Höhn, H. Stange, A. Müller, P. Kleinschmidt, S. Brückner, T. Hannappel: ACS Appl. Mater. Interfaces, 2015, 7, 9323.

[261] T. J. Grassman, M. R. Brenner, S. Rajagopalan, R. Unocic, R. Dehoff, M. Mills, H. Fraser, S. A. Ringel: Appl. Phys. Lett., 2009, 94, 232106.

[262] Y. Takagi, H. Yonezu, K. Samonji, T. Tsuji, N. Ohshima: J. Cryst. Growth, 1998, 187, 42.

[263] V. K. Dixit, T. Ganguli, T. K. Sharma, S. D. Singh, R. Kumar, S. Porwal, P. Tiwari, A. Ingale, S. M. Oak: J. Cryst. Growth, 2008, 310, 3428.

[264] K. A. Bell, M. Ebert, S. D. Yoo, K. Flock, D. E. Aspnes: J. Vac. Sci. Technol. A, 2000, $18,1184$.

[265] T. Soga, T. Jimbo, M. Umeno: J. Cryst. Growth, 1995, 146, 554.

[266] K. J. Bachmann, U. Rossow, N. Sukidi, H. Castleberry, N. Dietz: J. Vac. Sci. Technol. $B, \mathbf{1 9 9 6}, 14,3019$.

[267] X. Liu, I. K. Kim, D. E. Aspnes: J. Vac. Sci. Technol. B, 2007, 25, 1448.

[268] T. Soga, T. Suzuki, M. Mori, T. Jimbo, M. Umeno: J. Cryst. Growth, 1993, 132, 134.

[269] T. Suzuki, T. Soga, T. Jimbo, M. Umeno: J. Cryst. Growth, 1991, 115, 158.

[270] K. Werner, A. Beyer, J. Oelerich, S. Baranovskii, W. Stolz, K. Volz: J. Cryst. Growth, 2014, 405, 102.

[271] S. Chandola, J. Power, T. Farrell, P. Weightman, J. McGilp: Appl. Surf. Sci., 1998, 123-124, 233.

[272] O. Supplie, M. M. May, G. Steinbach, O. Romanyuk, F. Grosse, A. Nägelein, P. Kleinschmidt, S. Brückner, T. Hannappel: J. Phys. Chem. Lett., 2015, 6, 464.

[273] A. Beyer, J. Ohlmann, S. Liebich, H. Heim, G. Witte, W. Stolz, K. Volz: J. Appl. Phys., 2012, 111, 083534.

[274] G. Steinbach, M. Schreiber, S. Gemming: Nanosci. Nanotechnol. Lett., 2013, $5,73$.

[275] O. Romanyuk, O. Supplie, T. Susi, M. M. May, T. H: Phys. Rev. B, 2016, 94, 155309.

[276] A. Stegmüller, K. Werner, M. Reutzel, A. Beyer, P. Rosenow, U. Höfer, W. Stolz, K. Volz, M. Dürr, R. Tonner: Chem. Eur. J., 2016, in press, doi:10.1002/chem.201602418.

[277] W. A. Harrison, E. A. Kraut, J. R. Waldrop, R. W. Grant: Phys. Rev. B, 1978, 18, 4402. 
[278] T. Hanada, T. Yasuda, A. Ohtake, K. Hingerl, S. Miwa, K. Arai, T. Yao: Phys. Rev. B, 1999, 60, 8909.

[279] E. Liarokapis, D. Papadimitriou, J. Rumberg, W. Richter: phys. status solidi (b), 1999, $211,309$.

[280] R. del Sole, G. Onida: Phys. Rev. B, 1999, 60, 5523.

[281] U. Rossow, L. Mantese, D. Aspnes: Appl. Surf. Sci., 1998, 123, 237.

[282] A. Beyer, A. Stegmüller, J. O. Oelerich, K. Jandieri, K. Werner, G. Mette, W. Stolz, S. D. Baranovskii, R. Tonner, K. Volz: Chem. Mat., 2016, 28, 3265.

[283] T. Hannappel, O. Supplie, M. M. May, P. Kleinschmidt, O. Romanyuk: arXiv:1610.01758 [cond-mat.mtrl-sci], 2016.

[284] R. Fischer, W. T. Masselink, J. Klem, T. Henderson, T. C. McGlinn, M. V. Klein, H. Morkoç, J. H. Mazur, J. Washburn: J. Appl. Phys., 1985, 58, 374.

[285] J. P. Gowers: Appl. Phys. A, 1984, 34, 231.

[286] T. N. Thanh, C. Robert, A. Létoublon, C. Cornet, T. Quinci, E. Giudicelli, S. Almosni, N. Boudet, A. Ponchet, J. Kuyyalil, M. Danila, O. Durand, N. Bertru, A. L. Corre: Thin Solid Films, 2013, 541, 36.

[287] P. N. Uppal, H. Kroemer: J. Appl. Phys., 1985, 58, 2195.

[288] I. Németh, B. Kunert, W. Stolz, K. Volz: J. Cryst. Growth, 2008, 310, 1595.

[289] H. Döscher, B. Borkenhagen, G. Lilienkamp, W. Daum, T. Hannappel: Surf. Sci., 2011, 605, L38.

[290] B. Borkenhagen, H. Döscher, T. Hannappel, G. Lilienkamp, W. Daum: ECS Trans., 2012, 45, 231.

[291] M. Lei, J. Price, W.-E. Wang, M. H. Wong, R. Droopad, P. Kirsch, G. Bersuker, M. C. Downer: Appl. Phys. Lett., 2013, 102, 152103.

[292] J. Amzallag, H. Benisty, S. Debrus, M. May, M. Eddrief, A. Bourdon, A. Chevy, N. Piccioli: Appl. Phys. Lett., 1995, 66, 982.

[293] K. Brixius, A. Beyer, J. Güdde, M. Dürr, W. Stolz, K. Volz, U. Höfer: arXiv:1611.01307 [cond-mat.mes-hall], 2016.

[294] K. Ishioka, K. Brixius, A. Beyer, A. Rustagi, C. J. Stanton, W. Stolz, K. Volz, U. Höfer, H. Petek: Appl. Phys. Lett., 2016, 108, 051607.

[295] M. Kawabe, T. Ueda: Jap. J. Appl. Phys., 1987, 26, L944.

[296] I. Németh, B. Kunert, W. Stolz, K. Volz: J. Cryst. Growth, 2008, 310, 4763.

[297] A. Beyer, B. Haas, K. I. Gries, K. Werner, M. Luysberg, W. Stolz, K. Volz: Appl. Phys. Lett., 2013, 103, 032107.

[298] H. Döscher, O. Supplie, S. Brückner, T. Hannappel, A. Beyer, J. Ohlmann, K. Volz: J. Cryst. Growth, 2011, 315, 16.

[299] A. Navarro, P. C. E. García-Tabarés, B. Galiana, I. Rey-Stolle, C. Ballesteros: J. Cryst. Growth, 2016, 464, 8.

[300] E. García-Tabarés, I. Rey-Stolle: Sol. Energ. Mat. Sol. Cells, 2014, 124, 17.

[301] O. Supplie: Dissertation thesis, Humboldt-Universität zu Berlin, 2016, urn:nbn:de:kobv:11-100239508.

[302] U. Gelius, E. Basilier, S. Svensson, T. Bergmark, K. Siegbahn: J. Electr. Spectr. Rel. Phen., 1973, 2, 405.

[303] M. M. May, C. Brabetz, C. Janowitz, R. Manzke: Phys. Rev. Lett., 2011, 107, 176405.

[304] G. Chen, S. B. Visbeck, D. C. Law, R. F. Hicks: J. Appl. Phys., 2002, 91, 9362.

[305] D. Schmeisser, F. J. Himpsel, G. Hollinger: Phys. Rev. B, 1983, 27, 7813.

[306] M. A. Henderson: Surf. Sci. Rep., 2002, 46, 1.

[307] J. M. White, S. Akhter: Crit. Rev. Solid State Mater. Sci., 1988, 14, 131.

[308] I. Langmuir: J. Amer. Chem. Soc., 1918, 40, 1361.

[309] D. A. King, M. G. Wells: Surf. Sci, 1972, 29, 454.

[310] C. Powell, A. Jablonski: J. Electr. Spectr. Rel. Phen., 2010, 178-179, 331. 
[311] O. Henrion, A. Klein, W. Jaegermann: Surf. Sci., 2000, 457, L337.

[312] T. Bitzer, H. Lewerenz: Surf. Sci., 1992, 269-270, 886.

[313] K. E. Bean, P. S. Gleim: Proc. IEEE, 1969, 57, 1469.

[314] R. E. Blaha, W. R. Fahrner: phys. status solidi (a), 1978, 50, 551.

[315] S. Katircioglu: Surf. Sci., 1987, 187, 569.

[316] K. Fujiwara: Surf. Sci., 1981, 108, 124.

[317] W. Ranke, Y. Xing: Surf. Sci., 1997, 381, 1.

[318] N. Witkowski, R. Coustel, O. Pluchery, Y. Borensztein: Surf. Sci., 2006, 600, 5142.

[319] M. Dürr, U. Höfer: Surf. Sci. Rep., 2006, 61, 465.

[320] N. Sano, T. Shibata, T. Kanki, T. Asano: J. Chem. Eng..Jap., 2001, 34, 684.

[321] A. Heller, B. Miller, H. J. Lewerenz, K. J. Bachmann: J. Amer. Chem. Soc., 1980, 102, 6555.

[322] H. J. Lewerenz, C. Heine, K. Skorupska, N. Szabó, T. Hannappel, T. Vo-Dinh, S. A. Campbell, H. W. Klemm, A. G. Muñoz: Energy Environ. Sci., 2010, 3, 748.

[323] A. Baghbanpourasl, W. G. Schmidt, M. Denk, C. Cobet, M. Hohage, P. Zeppenfeld, K. Hingerl: Surf. Sci, 2015, 641, 231.

[324] B. C. Wood, E. Schwegler, W. I. Choi, T. Ogitsu: J. Phys. Chem. C, 2014, 118, 1062.

[325] J. L. Young, H. Döscher, J. A. Turner, T. G. Deutsch: J. Phys. Chem. C, 2016, 0, null.

[326] M. M. May, O. Supplie, C. Höhn, R. van de Krol, H.-J. Lewerenz, T. Hannappel: New J. Phys., 2013, 15, 103003.

[327] T. Letzig, F. Willig, P. H. Hahn, W. G. Schmidt: Phys. Rev. B, 2006, 74, 245307.

[328] C. X. Kronawitter, M. Lessio, P. Zhao, C. Riplinger, A. Boscoboinik, D. E. Starr, P. Sutter, E. A. Carter, B. E. Koel: J. Phys. Chem. C, 2015, 119, 17762.

[329] X. Zhang, S. Ptasinska: Phys. Chem. Chem. Phys., 2015, 17, 3909.

[330] S. Ladas, R. Imbihl, G. Ertl: Surf. Sci., 1988, 198, 42.

[331] T. Bürgi, F. Atamny, A. Knop-Gericke, M. Hävecker, T. Schedel-Niedrig, R. Schlögl, A. Baiker: Catal. Lett., 2000, 66, 109.

[332] E. Savinova, D. Zemlyanov, B. Pettinger, A. Scheybal, R. Schlögl, K. Doblhofer: Electrochimica Acta, 2000, 46, 175.

[333] J. K. Nørskov: Topics in Catalysis, 1994, 1, 385.

[334] O. Björneholm, M. H. Hansen, A. Hodgson, L.-M. Liu, D. T. Limmer, A. Michaelides, P. Pedevilla, J. Rossmeisl, H. Shen, T. Gabriele, E. Tyrode, M.-M. Walz, J. Werner, H. Bluhm: Chem. Rev., 2016, 116, 7698.

[335] C. Punckt, F. S. Merkt, H. H. Rotermund: New J. Phys., 2007, 9, 213.

[336] R. Memming, G. Schwandt: Angew. Chem. Internat. Ed., 1967, 6, 851.

[337] H. Gerischer: Surf. Sci., 1969, 18, 97.

[338] H. Gerischer: J. Electroanal. Chem. Interf. Electrochem., 1983, 150, 553.

[339] A. J. Bard, L. R. Faulkner: Electrochemical Methods: Fundamentals and Applications, Wiley, New York, 2. edition, 2001, ISBN 978-0-471-04372-0.

[340] D. Kolb, J. Schneider: Electrochimica Acta, 1986, 31, 929.

[341] E. Ponomarev, L. Peter: J. Electroanal. Chem., 1995, 397, 45.

[342] F. Dimroth, R. Beckert, M. Meusel, U. Schubert, A. W. Bett: Prog. Photovolt. Res. Applic., 2001, 9, 165.

[343] I. Miethe, K. Krischer: J. Electroanal. Chem., 2012, 666, 1.

[344] K.-i. Ataka, T. Yotsuyanagi, , M. Osawa: J. Phys. Chem., 1996, 100, 10664.

[345] C. I. Smith, P. Harrison, T. Farrell, P. Weightman: J. Phys. Cond. Matt., 2012, 24, 482002.

[346] C. I. Smith, P. Harrison, C. A. Lucas, Y. Grunder, S. D. Barrett, P. Weightman: J. Phys.: Cond. Matt., 2016, 28, 015005.

[347] M. M. Lerch, M. J. Hansen, W. A. Velema, W. Szymanski, B. L. Feringa: Nat. Comm., 2016, 7. 
[348] B. A. Grzybowski, W. T. S. Huck: Nat. Nanotechnol., 2016, 11, 584.

[349] H. Logtenberg, J. Areephong, J. Bauer, A. Meetsma, B. L. Feringa, W. R. Browne: ChemPhysChem, 2016, 17, 1895.

[350] Y. Yan, S. C. Warren, P. Fuller, B. A. Grzybowski: Nat. Nanotechnol., 2016, 11, 603.

[351] N. Koumura, R. W. J. Zijlstra, R. A. van Delden, N. Harada, B. L. Feringa: Nature, 1999, 401, 152.

[352] R. Eelkema, M. M. Pollard, J. Vicario, N. Katsonis, B. S. Ramon, C. W. M. Bastiaansen, D. J. Broer, B. L. Feringa: Nature, 2006, 440, 163.

[353] T. Kudernac, N. Ruangsupapichat, M. Parschau, B. Macia, N. Katsonis, S. R. Harutyunyan, K. H. Ernst, B. L. Feringa: Nature, 2011, 479, 208.

[354] R. Yoshida, T. Ueki: NPG Asia Mater., 2014, 6.

[355] H. W. H. van Roekel, B. J. H. M. Rosier, L. H. H. Meijer, P. A. J. Hilbers, A. J. Markvoort, W. T. S. Huck, T. F. A. de Greef: Chem. Soc. Rev., 2015, 44, 7465.

[356] S. N. Semenov, L. J. Kraft, A. Ainla, M. Zhao, M. Baghbanzadeh, V. E. Campbell, K. Kang, J. M. Fox, G. M. Whitesides: Nature, 2016, 537, 656.

[357] S. N. Semenov, A. S. Y. Wong, R. M. van der Made, S. G. J. Postma, J. Groen, H. W. H. van Roekel, T. F. A. de Greef, W. T. S. Huck: Nat. Chem., 2015, 7, 160.

[358] X. M. He, M. Aizenberg, O. Kuksenok, L. D. Zarzar, A. Shastri, A. C. Balazs, J. Aizenberg: Nature, 2012, 487, 21.

[359] M. Emond, T. L. Saux, J. F. Allemand, P. Pelupessy, R. Plasson, L. Jullien: Chem-Eur. J., 2012, 18, 14375.

[360] M. Kato, J. Z. Zhang, N. Paul, E. Reisner: Chem. Soc. Rev., 2014, 43, 6485.

[361] F. Caschera, V. Noireaux: Curr. Opin. Chem. Biol., 2014, 22, 85.

[362] M. Forgac: Nat. Rev. Mol. Cell. Bio., 2007, 8, 917.

[363] B. P. Pedersen, M. J. Buch-Pedersen, J. P. Morth, M. G. Palmgren, P. Nissen: Nature, 2007, 450, 1111.

[364] F. Rivera-Chavez, A. J. Baumler: Annu. Rev. Microbiol., 2015, 69, 31.

[365] E. V. Skorb, A. V. Volkova, D. V. Andreeva: Curr. Org. Chem., 2015, 19, 1097.

[366] E. V. Skorb, D. V. Andreeva: Adv. Funct. Mater., 2013, 23, 4483.

[367] E. V. Skorb, H. Möhwald: Adv. Mater., 2013, 25, 5029.

[368] S. A. Ulasevich, G. Brezesinski, H. Möhwald, P. Fratzl, F. H. Schacher, S. K. Poznyak, D. V. Andreeva, E. V. Skorb: Angew. Chem. Int. Ed., 2016, 55, 13001.

[369] D. V. Andreeva, I. Melnyk, O. Baidukova, E. V. Skorb: ChemElectroChem, 2016, 3.

[370] S. A. Ulasevich, N. Brezhneva, Y. Zhukova, H. Möhwald, P. Fratzl, F. H. Schacher, D. V. Sviridov, D. V. Andreeva, E. V. Skorb: Macromol. Biosci., 2016, 16, 1422.

[371] A. L. Linsebigler, G. Q. Lu, J. T. Yates: Chem. Rev., 1995, 95, 73.

[372] M. A. Henderson, I. Lyubinetsky: Chem. Rev., 2013, 113, 4428.

[373] E. V. Skorb, E. A. Ustinovich, A. I. Kulak, D. V. Sviridov: J. Photochem. Photobiol. A, 2008, 193, 97.

[374] K. Ichimura: Chem. Rec., 2002, 2, 46.

[375] S. A. Ulasevich, S. K. Poznyak, A. I. Kulak, A. D. Lisenkov, M. Starykevich, E. V. Skorb: Langmuir, 2016, 32, 4016.

[376] I. Richard, M. Thibault, G. D. Crescenzo, M. D. Buschmann, M. Lavertu: Biomacromol., 2013, 14, 1732.

[377] R. V. Benjaminsen, M. A. Mattebjerg, J. R. Henriksen, S. M. Moghimi, T. L. Andresen: Mol. Ther., 2013, 21, 149.

[378] E. V. Skorb, D. V. Andreeva: Polym. Int, 2015, 64, 713.

[379] D. V. Andreeva, E. V. Skorb, D. G. Shchukin: ACS Appl. Mater. Inter., 2010, 2, 1954.

[380] E. V. Skorb, H. Möhwald: Adv. Mater. Interfaces, 2014, 1.

[381] J. Schneider, M. Matsuoka, M. Takeuchi, J. L. Zhang, Y. Horiuchi, M. Anpo, D. W. Bahnemann: Chem. Rev., 2014, 114, 9919. 
[382] J. Gensel, T. Borke, N. P. Perez, A. Fery, D. V. Andreeva, E. Betthausen, A. H. E. Muller, H. Möhwald, E. V. Skorb: Adv. Mater., 2012, 24, 985.

[383] E. V. Skorb, L. I. Antonouskaya, N. A. Belyasova, D. G. Shchukin, H. Möhwald, D. V. Sviridov: Appl. Catal. B-Environ., 2008, 84, 94.

[384] C. D. Nadell, K. Drescher, K. R. Foste: Nat. Rev. Microbiol., 2016, 14, 589.

[385] G. M. Whitesides: Interface Focus, 2015, 5.

[386] S. E. Winter, P. Thiennimitr, M. G. Winter, B. P. Butler, D. L. Huseby, R. W. Crawford, J. M. Russell, C. L. Bevins, L. G. Adams, R. M. Tsolis, J. R. Roth, A. J. Baumler: Nature, 2010, 467, 426.

[387] M. Hensel, A. P. Hinsley, T. Nikolaus, G. Sawers, B. C. Berks: Mol. Microbiol., 1999, $32,275$.

[388] C. J. Huang, E. L. Barrett: J. Bacteriol., 1991, 173, 1544.

[389] N. K. Heinzinger, S. Y. Fujimoto, M. A. Clark, M. S. Moreno, E. L. Barrett: J Bacteriol., 1995, 177, 2813.

[390] C. A. Lopez, S. E. Winter, F. Rivera-Chavez, M. N. Xavier, V. Poon, S. P. Nuccio, R. M. Tsolis, A. J. Baumler: Mbio, 2012, 3.

[391] P. Louis, H. J. Flint: Fems Microbiol. Lett., 2009, 294, 1.

[392] E. V. Skorb, H. Möhwald, D. V. Andreeva: Adv. Mater. Interfaces, 2016, doi: 10.1002/admi.201600282.

[393] D. O. Grigoriev, K. Kohler, E. Skorb, D. G. Shchukin, H. Möhwald: Soft Matter, 2009, $5,1426$.

[394] K. J. M. Bishop, C. J. Campbell, G. Mahmud, B. A. Grzybowski: Stud. Multidiscip., 2008, 5, 21.

[395] E. V. Skorb, A. V. Volkova, D. V. Andreeva: Curr. Org. Chem., 2014, 18, 2315.

[396] D. B. Zorov, C. R. Filburn, L. O. Klotz, J. L. Zweier, S. J. Sollott: J. Exp. Med., 2000, 192, 1001.

[397] D. B. Zorov, M. Juhaszova, K. W. Fishbein, S. J. Sollott: Biophys. J., 2001, 80, 500.

[398] D. B. Zorov, M. Juhaszova, S. J. Sollott: Physiol. Rev., 2014, 94, 909.

[399] Y. B. Liu, G. Fiskum, D. Schubert: J. Neurochem., 2002, 80, 780.

[400] L. A. Munishkina, A. L. Fink: Bba-Biomembranes, 2007, 1768, 1862.

[401] W. E. Moerner, D. P. Fromm: Rev. Sci. Instrum., 2003, 74, 3597.

[402] P. Tinnefeld, M. Sauer: Angew. Chem. Int. Ed., 2005, 44, 2642.

[403] M. Hayyan, M. A. Hashim, I. M. AlNashef: Chem. Rev., 2016, 116, 3029.

[404] I. Johnson (Ed.), M.T.Z. Spence (Ed.): The Molecular Probes Handbook. A Guide to Fluorescent Probes and Labeling Technologies, 11th Edition, Thermo Fisher Scientific, 2010, ISBN: 0982927916.

[405] A. Gomes, E. Fernandes, J. L. F. C. Lima: J. Biochem. Bioph. Meth., 2005, 65, 45.

[406] J. Suzurikawa, M. Nakao, R. Kanzaki, H. Takahashi: Sensor Actuat B-Chem., 2010, 149, 205.

[407] D. Fix, E. V. Skorb, D. G. Shchukin, H. Möhwald: Meas. Sci. Technol., 2011, 22.

[408] J. Kopf, S. Ulasevich, O. Baidukova, Y. Zhukova, J. W. C. Dunlop, P. Fratzl, P. Rikeit, P. Knaus, S. K. Poznyak, D. V. Andreeva, E. V. Skorb: Adv. Eng. Mater., 2016, 18, 476.

[409] E. V. Skorb, D. V. Andreeva, H. Möhwald: Angew. Chem. Int. Ed., 2012, 51, 5138.

[410] F. Priolo, T. Gregorkiewicz, M. Galli, T. F. Krauss: Nat. Nanotechnol., 2014, 9, 19.

[411] J. D. Holmes, K. P. Johnston, R. C. Doty, B. A. Korgel: Science, 2000, 287, 1471.

[412] L. T. Canham: Nature, 2000, 408, 411.

[413] E. V. Skorb, H. Möhwald: Ultrason. Sonochem., 2016, 29, 589.

[414] E. V. Skorb, H. Möhwald, D. V. Andreeva: Langmuir, 2016.

[415] K. Sunada, T. Watanabe, K. Hashimoto: J. Photochem. Photobiol. A, 2003, 156, 227. 
[416] E. V. Skorb, D. V. Andreeva, A. P. Raiski, N. A. Belyasova, H. Möhwald, D. V. Sviridov: Photochem. Photobiol. Sci., 2011, 10, 1974.

[417] J. A. Byrne, P. S. M. Dunlop, J. W. J. Hamilton, P. Fernandez-Ibanez, I. Polo-Lopez, P. K. Sharma, A. S. M. Vennard: Molecules, 2015, $20,5574$.

[418] Y. Paz, A. Heller: J. Mater. Res., 1997, 12, 2759.

[419] P. S. Foran, C. Boxall, K. R. Denison: Langmuir, 2012, 28, 17647.

[420] E. V. Skorb, A. G. Skirtach, D. V. Sviridov, D. G. Shchukin, H. Möhwald: ACS Nano, 2009, 3, 1753.

[421] E. V. Skorb, D. G. Shchukin, H. Möhwald, D. V. Sviridov: J. Mater. Chem., 2009, 19, 4931.

[422] B. W. Liu, X. Y. Yu, Z. H. Zhu, X. Hua, L. Yang, Z. Y. Wang: Lab Chip, 2014, 14, 855.

[423] E. V. Skorb, D. V. Sviridov, H. Möhwald, D. G. Shchukin: Chem. Comm., 2000, page 6041.

[424] E. V. Skorb, D. Fix, D. V. Andreeva, H. Möhwald, D. G. Shchukin: Adv. Funct. Mater., 2009, 19, 2373.

[425] E. V. Skorb, O. Baidukova, A. Goyal, A. Brotchie, D. V. Andreeva, H. Möhwald: J. Mater. Chem., 2012, 22, 13841.

[426] D. V. Andreeva, D. V. Sviridov, A. Masic, H. Möhwald, E. V. Skorb: Small, 2012, 8, 820.

[427] M. Rogoz, H. Zeng, C. Xuan, D. S. Wiersma, P. Wasylczyk: Adv. Opt. Mater., 2016, doi: 10.1002/adom.201600503.

[428] M. Su, V. P. Dravid: Nano Lett., 2005, 5, 2023.

[429] R. F. Dong, Q. L. Zhang, W. Gao, A. Pei, B. Y. Ren: ACS Nano, 2016, 10, 839.

[430] S. J. Park, M. Gazzola, K. S. Park, S. Park, V. D. Santo, E. L. Blevins, J. U. Lind, P. H. Campbell, S. Dauth, A. K. Capulli, F. S. Pasqualini, S. Ahn, A. Cho, H. Y. Yuan, B. M. Maoz, R. Vijaykumar, J. W. Choi, K. Deisseroth, G. V. Lauder, L. Mahadevan, K. K. Parker: Science, 2016, 353, 158.

[431] M. Wehner, R. L. Truby, D. J. Fitzgerald, B. Mosadegh, G. M. Whitesides, J. A. Lewis, R. J. Wood: Nature, 2016, 536, 451.

[432] Editorial: Nat. Mater., 2012, 11, 91.

[433] I. Vurgaftman, J. R. Meyer, L. R. Ram-Mohan: J. Appl. Phys., 2001, 89, 5815.

[434] T. E. Fischer: Phys. Rev., 1966, 142, 519.

[435] W. N. Hansen, D. M. Kolb: J. Electroanal. Chem. Interfac., 1979, 100, 493.

[436] S. Tiwari, D. J. Frank: Appl. Phys. Lett., 1992, 60, 630.

[437] American Society for Testing and Materials (ASTM): Terrestrial reference spectra for photovoltaic performance evaluation.

[438] M. M. May, D. Lackner, J. Ohlmann, F. Dimroth, R. van de Krol, T. Hannappel, K. Schwarzburg: Sustainable Energy \& Fuels, 2017, 1, 492.

[439] P. Lautenschlager, M. Garriga, M. Cardona: Phys. Rev. B, 1987, 36, 4813.

[440] S. Zollner, M. Garriga, J. Kircher, J. Humlicek, M. Cardona, G. Neuhold: Phys. Rev. B, 1993, 48, 7915.

[441] P. Lautenschlager, M. Garriga, L. Vina, M. Cardona: Phys. Rev. B, 1987, 36, 4821.

[442] J. D. E. McIntyre, D. E. Aspnes: Surf. Sci., 1971, 24, 417.

[443] A. Mishra, J. S. Kwon, R. Thakur, S. Wereley: Trends Biotechnol., 2014, 32, 415.

[444] A. A. Solovev, W. Xi, D. H. Gracias, S. M. Harazim, C. Deneke, S. Sanchez, O. G. Schmidt: ACS Nano, 2012, 6, 1751.

[445] C. Kim, H. J. Park, S. Cha, J. Yoon: Chemosphere, 2013, 93, 2011.

[446] Y. Li, W. Zhang, J. F. Niu, Y. S. Chen: ACS Nano, 2012, 6, 5164.

[447] W. Zhang, Y. Li, J. F. Niu, Y. S. Chen: Langmuir, 2013, 29, 4647. 
[448] C. Kormann, D. W. Bahnemann, M. R. Hoffmann: Environ. Sci. Technol., 1988, 22, 798.

[449] K. Ishibashi, A. Fujishima, T. Watanabe, K. Hashimoto: J. Photochem. Photobiol. A, $2000,134,139$.

[450] Q. Xiao, Z. C. Si, J. Zhang, C. Xiao, X. K. Tan: J. Hazard. Mater., 2008, 150, 62.

[451] L. M. Liu, W. Z. Sun, W. Y. Yang, Q. Li, J. K. Shang: Sci Rep-Uk, 2016, 6.

[452] B. H. Simpson, J. Rodriguez-Lopez: J. Am. Chem. Soc., 2015, 137, 14865.

[453] B. H. Simpson, J. Rodriguez-Lopez: Electrochim. Acta, 2015, 179, 74.

[454] H. S. Park, K. C. Leonard, A. J. Bard: J. Phys. Chem. C, 2013, 117, 12093.

[455] H. Sakai, R. Baba, K. Hashimoto, A. Fujishima, A. Heller: J. Phys. Chem., 1995, 99, 11896.

[456] T. Hirakawa, H. Kominami, B. Ohtani, Y. Nosaka: J. Phys. Chem. B, 2001, 105, 6993.

[457] D. B. Wang, L. X. Zhao, L. H. Guo, H. Zhang: Anal. Chem., 2014, 86, 10535.

[458] Q. J. Xiang, J. G. Yu, P. K. Wong: J. Colloid. Interf. Sci., 2011, 357, 163.

[459] K. Ishibashi, A. Fujishima, T. Watanabe, K. Hashimoto: Electrochem. Commun., 2000, 2, 207.

[460] S. Kohtani, K. Yoshida, T. Maekawa, A. Iwase, A. Kudo, H. Miyabe, R. Nakagaki: Phys. Chem. Chem. Phys., 2008, 10, 2986.

[461] H. Li, Y. Tian, Z. F. Deng, Y. Liang: Analyst, 2012, 137, 4605.

[462] R. Nakamura, T. Okamura, N. Ohashi, A. Imanishi, Y. Nakato: J. Am. Chem. Soc., 2005, 127, 12975 .

[463] T. Tachikawa, T. Majima: Chem. Soc. Rev., 2010, 39, 4802.

[464] K. Naito, T. Tachikawa, M. Fujitsuka, T. Majima: J. Am. Chem. Soc., 2009, 131, 934.

[465] T. Tachikawa, T. Majima: J. Fluoresc., 2007, 17, 727.

[466] K. Naito, T. Tachikawa, M. Fujitsuka, T. Majima: J. Phys. Chem. C, 2008, 112, 1048.

[467] J. Z. Ma, H. M. Wu, Y. C. Liu, H. He: J. Phys. Chem. C, 2014, 118, 7434.

[468] K. Fujiwara, U. Muller, S. E. Pratsinis: ACS Catal., 2016, 6, 1887.

[469] G. H. Dong, W. K. Ho, L. Z. Zhang: Appl. Catal. B-Environ., 2015, 168, 490.

[470] A. Alonso-Tellez, D. Robert, V. Keller, N. Keller: Environ. Sci. Pollut. R, 2014, 21, 3503.

[471] A. E. Raevskaya, A. L. Stroyuk, S. Y. Kuchmii: J. Nanopart. Res., 2004, 6, 149. 
$\underline{\text { Table of content entry: }}$

Interfaces play a decisive role in efficient solar energy conversion and photo-active sensors. Gaining thorough insight into interfacial reactions, however, is particularly challenging in relevant complex, non-vacuum environments. This review focuses on in situ studies of interface formation during preparation in chemical vapor ambient, modification in gaseous and liquid environment and coupling of inorganic photoactive material with bioactive soft matter.

Keywords: interfaces, energy conversion, in situ analysis, biosensors, photo-induced reactions

O. Supplie, M.M. May, S. Brückner, N. Brezhneva, T. Hannappel*, E.V. Skorb*

In situ characterization of interfaces relevant for efficient photo-induced reactions

ToC figure ((Please choose one size: $55 \mathrm{~mm}$ broad $\times 50 \mathrm{~mm}$ high or $110 \mathrm{~mm}$ broad $\times 20 \mathrm{~mm}$ high. Please do not use any other dimensions))

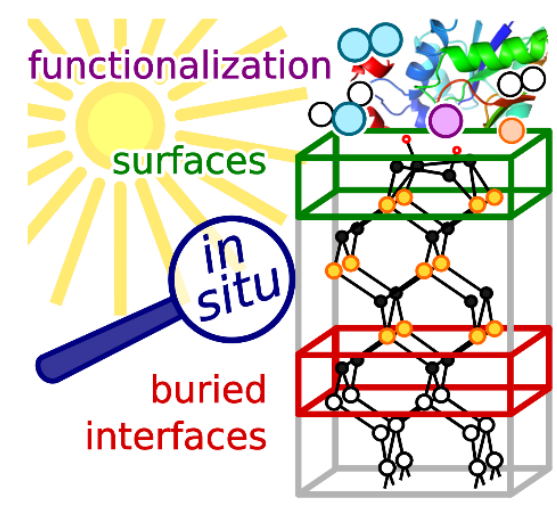

